\title{
Versicherungs-Statistik für 1915
}

\section{Einleitung}

Die Veröffentlichung der Versicherungs-Statistik für 1915 hat sich aus denselben Gründen wie die der beiden vorhergehenden Jahre stark verzögert: die nicht zur Fahne einberufenen Beamten mußten in erster Linie die durch den Krieg bedingten besonderen :Geschäfte und die laufenden Geschäfte für die unmittelbare Aufsichtsführung erledigen; die Statistik mußte als die weniger dringliche Aufgabe zurückstehen. Wenn in den nächsten Jahren wieder mit allen erforderlichen Kräften wird gearbeitet werden können, wird die Verspätung im Erscheinen der Statistik allmählich wieder beseitigt werden.

Unter Reichsaufsicht stehen 1. diejenigen inländischen privaten Versicherungsunternehmungen, deren Geschäftsbetrieb nicht durch die Satzung oder die sonstigen Geschäftsunterlagen auf das Gebiet eines Bundesstaats beschränkt ist ( 22 des Versicherungsaufsichtsgesetzes), soweit sie nicht bei sachlich, örtlich oder hinsichtlich des Personenkreises eng begrenzter Ausdehnung nach $\S 3$ Abs. 2 a. a. O. der Aufsicht einer Landesbehörde überwiesen worden sind, 2. diejenigen inländischen privaten Unternehmungen, deren Geschäftsbetrieb auf das Gebiet eines Bundesstaats beschränkt ist, deren Beaufsichtigung aber auf Grund von $\S 3$ Abs. 1 a. a. O. dem Aufsichtsamt übertragen ist, 3. die ausländischen Versicherungsunternehmungen, die im Inlande durch Vertreter, Bevollmächtigte, Agenten oder sonstige Vermittler das Versicherungsgeschäft betreiben (\$91 a. a. 0.), 4. in besehränktem Umfange diejenigen inländischen privaten Versicherungsunternehmungen, welche die Rückversicherung in gesetzlich aufsichtspflichtigen Versicherungszweigen zum Gegenstande haben (Bekanntmachung vom 18. Juni 1908, R.G.Bl. S. 409).

Ausgenommen von der Aufsicht waren im Berichtsjahre 1. Unternehmungen, welche die Versicherung gegen Kursverluste oder die Transportversicherung betreiben ( $\$ 116$ a. a. O), 2. die auf Grund landesrechtlicher Vorschriften errichteten Hilfskassen, die auf Grund der Gewerbeordnung von Innungen oder Innungsverbänden errichteten Unterstützungskassen sowie die auf Grund berggesetzlicher Vorschriften errichteten Knappschaftskassen (§ 122 a. a. O.).

Grundsätzlich ausgeschlossen von unserer Statistik sind von den der Reichsaufsicht unterstehenden Unternehmungen diejenigen, deren Beaufsichtigung auf Grund des erwähnten $§ 3$ Abs. 1 dem Aufsichtsamt überwiesen ist; es waren das im Jahre I9I 5 279 Bayerische, 658 Hessische, 1 Mecklenburg-Strelitzscher, 81 Schaumburg-Lippische und 92 Lippische Versicherungsvereine, die ihren Geschäftsbetrieb über ihren Heimatsstaat nicht hinauserstrecken. Wenn ihre Zahl auch groß ist, so fallen sie ihrer Be- 
deutung nach doch wenig ins Gewicht; das zeigen z. B. die Zusammenstellungen, die in den Geschäftsberichten des Aufsichtsamts seit dem Jahre I905 regelmäßig gegeben sind. Ebenso sind ausgeschlossen die Krankenkassen, die infolge Aufhebung des Hilfskassengesetzes mit dem I. Juni 1912 unter Reichsaufsicht gekommen sind.

Fortgelassen sind auch die belgischen, die englischen und die französischen Gesellschaften, die in Friedenszeiten in Deutschland arbeiteten, weil sie sehr bald nach Kriegsausbruch neueVersioherungen in Deutschland nicht mehr abgeschlossen und haben ordnungsmäßige Rechnungsabschlüsse während des Krieges von ihnen nicht zu beschaffen waren.

Der in den Tafeln zusammengestellte Zahlenstoff beruht auf den Jahresabschlüssen, die dem Amte gemäß seinen "Vorschriften über die Rechnungslegung der vom Reichsaufsichtsamte für Privatversicherung beaufsichtigten größeren Versicherungsunternehmungen " (Ver. A. f. P. 1902 S. 23 ff.) und "Vorschriften über die Rechnungslegung der vom Reichsaufsichtsamte für Privatversicherung beaufsichtigten Rückversicherungsunternehmungen “ (Ver. A.f. P. 1909 S. 2 ff.) eingereicht werden. Als Geschäftsjahr gilt bei der überwiegenden Mehrzahl der Unternehmungen das Kalenderjahr; wo das nicht der Fall ist, bezieht sich diese Statistik auf dasjenige Geschäftsjahr, dessen Beginn in das Jahr 1915 fallt.

Die Nachprüfung der Abschlüsse für das Geschäftsjahr 1915 hat einige Zahlen der vorjährigen Abschlüsse als unrichtig erwiesen, die bei der Bearbeitung der Statistik für 1914 nach den Berichten der Unternehmungen für richtig gehalten werden mußten. Diese Zahlen sind in den Anmerkungen zu den Tafeln für 1915 gekennzeichnet und außerdem unter den Berichtigungen am Schlusse dieses Bandes aufgeführt.

Die Anordnung des Stoffes ist dieselbe wie in den früheren Statistiken.

Die sämtlichen Unternehmungen sind nach der Art ihres Betriebs in fünf Gruppen eingeteilt, nämlich:

1. Lebensversicherung und Krankenversicherung,

2. Unfall- und Haftpflichtversicherung,

3. Viehversicherung, Hagelversicherung und sonstige landwirtschaftliche Versicherung,

4. Feuerversicherung (einschl. der Versicherung mittelbarer Schäden), Versicherung gegen Sturmschäden, Wasserschäden und Diebstahl,

5. Sonstige Versicherungszweige (darunter Glasversicherung und Rückversicherung).

Innerhalb dieser Gruppen sind die Unternehmungen nach den von ihnen betriebenen Versicherungszweigen getrennt; sie folgen einander aber in allen Tafeln in derselben Reihe und stets unter derselben Nummer.

Zur Veranschaulichung der Entwickelung der einzelnen Versicherungszweige in den letzten fünf Jahren ist der Statistik eine Tafel beigegeben. 


\section{Allgemeiner Überblick}

Diese Statistik berichtet eingehend über 251 deutsche und 34 ausländische Unter- Die Unter nehmungen. Von den deutschen Unternehmungen sind 115 Aktiengesellschaften; die übrigen beruhen, abgesehen von wenigen Einzelunternehmern und Genossenschaften mit beschränkter Haftpflicht, auf dem Grundsatze der Gegenseitigkeit.

Die Gegenseitigkeitsvereine beschränkten ihren Betrieb fast alle auf einen einzelnen Geschäftszweig; nur $\left.16(14)^{*}\right)$ von ihnen betätigten sich gleichzeitig in verschiedenen Versicherungszweigen; alle arbeiteten vornehmlich unmittelbar, kein Verein betrieb lediglich Rückversicherung. Von den 115 Aktiengesellschaften waren 35 (33) ausschließliche Rückversicherungsunternehmungen, sämtlich mit mehreren Zweigen; von den übrigen, auch unmittelbar arbeitenden 80 (81) Aktiengesellschaften pflegten 55 (55) mehrere Zweige. Die kleineren Geschäftszweige: die Sturmschäden-, die Wasserleitungsschäden-, die Glas-, die Kautions-, Bürgschafts-, Kredit- sowie die Maschinenversicherung, aber auch die Unfall- und Haftpflichtversicherung werden fast ausschließlich von Aktiengesellschaften gepflegt. Die seit dem Jahre I 9 I I nachgewiesenen Versicherungen mittelbarer Schäden (Mietverlust-, Betriebsverlust- und Zuckerpreisdifferenzversicherung), die im Zusammenhange mit der Feuer- oder der Wasserleitungsschädenversicherung betrieben werden, sind nicht als besondere Geschäftszweige anzusehen.

Von den in dieser Statistik ausführlich behandelten unmittelbar versichernden deutschen Unternehmungen haben im Berichtsjahre gearbeitet in der Lebensversicherung 30 (30) Aktiengesellschaften und 32 (33) Gegenseitigkeitsvereine, in der Unfallversicherung 30 (30) Aktiengesellschaften und 1 (1) Gegenseitigkeitsverein, in der Haftptlichtversicherung 30 (30) Aktiengesellschaften und 1 (1) Verein, in der Viehversicherung 1 (1) Aktiengesellschaft und 26 (27) Gegenseitigkeitsvereine, in der Hagelversicherung 4 (4) Aktiengesellschaften (sämtlich mit Ausschluß anderer Versicherungsgeschäfte) und 12 (12) Gegenseitigkeitsvereine (von denen drei auch die Feuerversicherung und einer auch die Baulastversicherung betrieben), in der Feuerversicherung 40 (41) Aktiengesellschaften und 24 (22) Gegenseitigkeitsvereine, in der Versicherung gegen Einbruchdiebstahl 43 (44) Aktiengesellschaften und 13 (11) Gegenseitigkeitsvereine, und endlich in der Glasversicherung 21 (21) Aktiengesellschaften und 2 (2) Gegenseitigkeitsvereine. Bei 37 (37) Kaskoversicherungsunternehmungen waren am Schlusse des Berichtsjahrs aber 8300 (8700) Fahrzeuge im Werte von 98,19 $(103,22)$ Millionen Mark versichert.

Auch die 35 (33) Rückversicherungsgesellschaften haben sich mit allen aufsichtspflichtigen Versicherungszweigen befaßt, und zwar (abgesehen von den weniger wichtigen Zweigen): 16 (16) mit der Lebensversicherung, 17 (17) mit der Unfallversicherung, 16 (15) mit der Haftpflichtversicherung, 4 (5) mit der Vieh- und 4 (3) mit der Hagelversicherung, 34 (32) mit der Feuerversicherung, 26 (23) mit der Einbruchdiebstahlversicherung und 8 (10) mit der Glasversicherung.

Die Zahl der in dieser Statistik behandelten ausländischen Gesellschaften stellt sich wie im Vorjahre wiederum auf nur 34, weil die englischen und die französischen Unternehmungen fortgelassen sind. Von den berücksichtigten hatten ihren Hauptsitz: 4 in den Vereinigten Staaten von Amerika, 1 in Belgien, 2 in Dänemark, 4 in den Niederlanden,

*) Die eingeklammerten Zahlen in diesem allgemeinen Uberblicke beziehen sich auf das vorige Jahr. 
12 in Osterreich, 2 in Schweden und 9 in der Schweiz. Gearbeitet haben 17 in der Lebensversicherung, -8 in der Unfall- und 6 in der Haftpflichtversicherung, 10 in der Feuerversicherung, 12 in der Einbrachdiebstahlversicherung, 3 in der Glasversicherung, 2 in der Kautions- und Veruntreuungsversicherung, 3 in der Wasserleitungsschädenund 1 in der Maschinenversicherung.

Umfang des

Gesobifts

Der Umfang des Versicherungsgeschäfts der auch unmittelbar arbeitenden deutschen Unternehmungen im Jahre 1915 wird durch die folgenden Ubersichten veranschaulicht, welche wegen der tiefgehenden Verschiedenheit der Grundlagen die Lebensversicherung einerseits und die übrigen Versicherungszweige anderseits getrennt behandeln.

\section{Umfang des Geschäfts der deutschen Versicherungsunternehmungen}

a) Lebensversicherung

\begin{tabular}{|c|c|c|c|c|c|c|c|c|}
\hline \multirow{2}{*}{ Versicherungsart } & \multirow{2}{*}{$\begin{array}{l}\text { Zahl der } \\
\text { Versiche- } \\
\text { rungs- } \\
\text { scheine }\end{array}$} & \multirow{2}{*}{$\begin{array}{c}\text { Versiche- } \\
\text { rungs- } \\
\text { summen } \\
1000 \mathscr{M}\end{array}$} & \multicolumn{4}{|c|}{$\begin{array}{c}\text { Zunahme (Abnahme -) } \\
\text { gegen das Vorjahr }\end{array}$} & \multicolumn{2}{|c|}{$\begin{array}{l}\text { Durchschnittlicher } \\
\text { Betrag eines Ver- } \\
\text { sicherungsscheins }\end{array}$} \\
\hline & & & $\begin{array}{l}\text { Versiche- } \\
\text { rungs- } \\
\text { scheine }\end{array}$ & $\%$ & $\begin{array}{l}\text { Summen } \\
1000 \mathscr{M}\end{array}$ & $\%$ & $\begin{array}{c}\text { Ende } \\
1915 \\
\mathscr{M}\end{array}$ & $\begin{array}{c}\text { Ende } \\
1914 \\
\mathscr{M} \\
\end{array}$ \\
\hline Versicherung auf den Todesfall... & 2680444 & 13269076 & $-8 I 08 I$ & $-2,94$ & $-2798 \times 3$ & $-2,06$ & $495^{\circ}$ & 4906 \\
\hline Versicherung auf den Lebensfall .. & 375255 & 640425 & -37542 & $-9,09$ & -55278 & $-7,95$ & 1707 & 1685 \\
\hline Rentenversicherung (Jahresienten) . & 72742 & 31164 & $-\quad 1637$ & $-2,20$ & -712 & $-2,23$ & 428 & 429 \\
\hline Volksversicherung $\ldots \ldots \ldots \ldots \ldots$ & 7914962 & I 536457 & -318559 & $-3,87$ & -88875 & $-5,47$ & 194 & 197 \\
\hline Zeitungsabonnentenversicherung ... & I 587372 & $13305^{8}$ & -26580 & $-1,65$ & $-\quad 2270$ & $-1,68$ & 84 & 84 \\
\hline Sonstige kleine Versicherung .. & 760444 & 413413 & $-\quad 15189$ & $-1,96$ & -10254 & $-2,42$ & 544 & 546 \\
\hline
\end{tabular}

Vergleicht man diese Zahlen mit denen des Vorjahrs, so findet man bei der Versicherung auf den Lebensfall, daß die Abnahme bei der Zahl der Versicherungsscheine wie bei, den Versicherungssummen etwas größer geworden ist; der durchschnittlich auf einen Schein entfallende Betrag ist fortdauernd, obwohl langsam, gewachsen: von $1570 \mathscr{M}$ im Jahre 1902 auf $1707 \mathscr{M}$ im Berichtsjahre. Die Ursache des fortdauernden Rückganges dieser Versicherungsart ist darin zu suchen, daß die Aussteuerversicherung jetzt vielfach durch die Todesfallversicherung mit Bonifikation im Erlebensfalle, die gemischte Versicherung und die Versicherung mit festem Auszahlungstermin, Versicherungsarten, die zu der Todesfallversicherung gerechnet werden, ersetzt wird. Wenn man also findet, daß alle anderen Versicherungsarten an Umfang zugenommen haben, so hängt das zum Teil auch mit dem Rückgange der Versicherung auf den Lebensfall zusammen. - Der Zuwachs der Volksversicherung hat allmählich nachgelassen. Bis einschließlich 1904 belief er sich bei den Scheinen und bei den Summen auf ungefähr 11 v. H. des Bestandes zu Anfang des Jahres; seitdem ist er bis 1912 bei den Summen auf 6,19 v. H., bei den Zahlen der Scheine auf 4,10 v. H. gesunken; 1913 schien sich wieder eine Hebung einleiten zu wollen, als in 1914 der Krieg mit seinen zahlreichen Todesfällen einen Rückschlag brachte, der sich auf 2,78. v. H. bei den Summen und auf 1,51 v. H. bei den Scheinen stellte. Im Berichtsjahre hat dieser Rückgang bereits 5,47 v. H. bei den Summen und 3,87 v. H. bei den Scheinen betragen. Die durchschnittliche Versicherungssumme hat zwischen 175 und $200 \mathscr{M}$ geschwankt. - Bei der großen Todesfallversicherung hat der Krieg die Zunahme der Scheine von 1,14 v. H. auf einen Rückgang von 2,94 v. H. und die der Summen von 1,58 v. H. auf einen Rückgang von 2,06 v. H. herabgedrückt. 
Der durchschnittlich auf einen Schein kommende Betrag ist fortdauernd gewachsen: von $4533 \mathscr{M}$ zu Ende I 903 auf $4950 \mathscr{M}$ am Ende des Berichtsjahrs. - Beim Rentengeschäft hat der Zuwachs betragen in Hundertsteln des Bestandes zu Anfang des Jahres

$\begin{array}{rrrrrr}\text { im Jahre } \ldots \ldots \ldots & 1911 & 1912 & 1913 & 1914 & 1915 \\ \text { bei der Zahl der Scheine................... } & 2,61 & -6,12 & -0,07 & -1,14 & -2,20 \\ \text { bei dem Jahresbetrage der versicherten Renten.. } & 4,79 & -3,91 & 1,03 & 0,78 & -2,23\end{array}$

Im Berichtsjahre sind von den Versicherten an Beiträgen und Ausfertigungsgebühren an die Unternehmungen gezahlt worden : in der Volksversicherung einschließlich derZeitungsabonnentenversicherung 118,70 $(129,26)$ Millionen Mark und in der großen Versicherung einschließlich der "sonstigen kleinen Versicherung " 594,64 $(618,81)$ Millionen Mark; in demselben Jahre sind für eingetretene Versicherungsfälle und für vorzeitig aufgelöste Versicherungen in der Volksversicherung ohne die mit den Summen ausgezahlten Gewinnanteile 62,12 (53,12) Millionen Mark und in der großen Versicherung 456,13 $(435,90)$ Millionen Mark bar an die Versicherten zurückgeflossen und außerdem 30,11 $(36,65)$ Millionen Mark und 176,72 $(195,61)$ Millionen Mark, überwiegend aus den Beitragseinnahmen, den Prämienreserven zugeführt worden. Die Zahlungen für Versicherungsfälle geben kein richtiges Bild für die tatsächliche Belastung, weil die Gesellschaften die Kriegstodesfälle verschieden behandeln. Einige zahlen sofort die volle Summe, andere sofort nur das Deckungskapital oder einen darüber hinausgehenden Teil der Versicherungssumme und machen eine Ergänzung nach dem Kriege von dem Stande ihrer freien Mittel oder von einer Umlage abhängig. Einen befriedigenden Uberblick ưber die Leistungen der Unternehmungen für Kriegstodesfälle wird man erst gewinnen, wenn diese Verhältnisse vollständig abgewickelt sein werden.

Bei den anderen Zweigen ist die Entwickelung auch in Friedenszeiten weniger gleichmäßig gewesen wie bei der Lebensversicherung. Immerhin hat die Unfallversicherung seit Jahren dauernd an Umfang gewonnen, erst der Krieg hat in 1914 einen Rückgang von 1,2 v. H. in der Zahl der Versicherungen gebracht, im Berichtsjahre sogar einen solchen von 14,9 v. H. Die Haftpflichtversicherung ist auch 1914 noch gewachsen, allerdings nur noch um 2,6 v. H., während sie in den beiden vorhergehenden Jahren um 6,9 und 6,8 v. H. zugenommen hatte. Im Berichtsjahre aber ist ein Rückgang von 2,3 v. H. eingetreten.

Der Umfang der Viehversicherung, an den Summen gemessen, hatte 1914 um 0,9 v. H. des vorjährigen Bestandes abgenommen; 1915 ist eine nicht viel größere $\mathrm{Ab}$ nahme, nämlich von 1,3 v. I., zu verzeichnen. Die Hagelversicherung unterliegt naturgemäß größern Schwankungen; im Vorjahre hatte die Zahl der Versicherungen um 0,6 v. H. und die Versicherungssumme um 1, $\dot{6}$ v. H. zugenommen, jetzt hat der Zugang 1,9 v. H. bzw. 11,5 v. H. des vorjährigen Bestandes betragen.

Die bis zum Ausbruch des Krieges im großen und ganzen gleichmäßige Entwickelung des Geschäfts der deutschen Feuerversicherungsunternehmungen hatte in I9I4 zum Teil dadurch eine Steigerung erfahren, daß infolge der Haftungs- und Uberführungsverträge, die zwischen den bei uns bis dahin tätigen englischen und französischen Gesellschaften und deutschen Unternehmungen vereinbart wurden (vgl. Veröffentlichungen des A.f.P. 1915 S. 78), zahlreiche bei ausländischen Gesellschaften laufende Versicherungen auf deutsche Gesellschaften übergingen. Der Versicherungsbestand der deutschen Unternehmungen stellte sich Ende 19 I4 bei der Zahl der Versicherungen um 4,6 v. H. und bei der Versicherungssumme um 5,9 v. H. höher als Ende I9I 3 und hatte damit 
ein wenig mehr zugenommen als in den vorhergehenden Friedensjahren. Jetzt im Berichtsjahre ist aber der Zugang wieder erheblich geringer geworden. Er stellt sich nur noch auf $1,5 \mathrm{v} . \mathrm{H}$. bei den Scheinen und auf $0,5 \mathrm{v}$. H. bei den Summen.

Von dem gesamten bei den deutschen Lebensversicherungsgesellschaften Ende 1915 (I 9 I 4) versicherten Kapitale, 15 99.2,43 $(16428,92)$ Millionen Mark, kommen 14440,67 $(14812,49)$ Millionen Mark auf das deutsche und 1 551,76 $(1616,43)$ Millionen Mark auf das ausländische Geschäft; dagegen hatten die ausländischen Lebensversicherungsgesellschaften zu derselben Zeit im Deutschen Reiche 1058,55 (1 101,66) Millionen Mark versichert; ihr Anteil an dem gesamten deutschen Kapitalversicherungsgeschäfte, 15499,22 $(15914,15)$ Millionen Mark, stellt sich danach auf $6,8(6,9)$ v. H.

b) Schadenversicherung In den auch unmittelbar betriebenen Zweigen

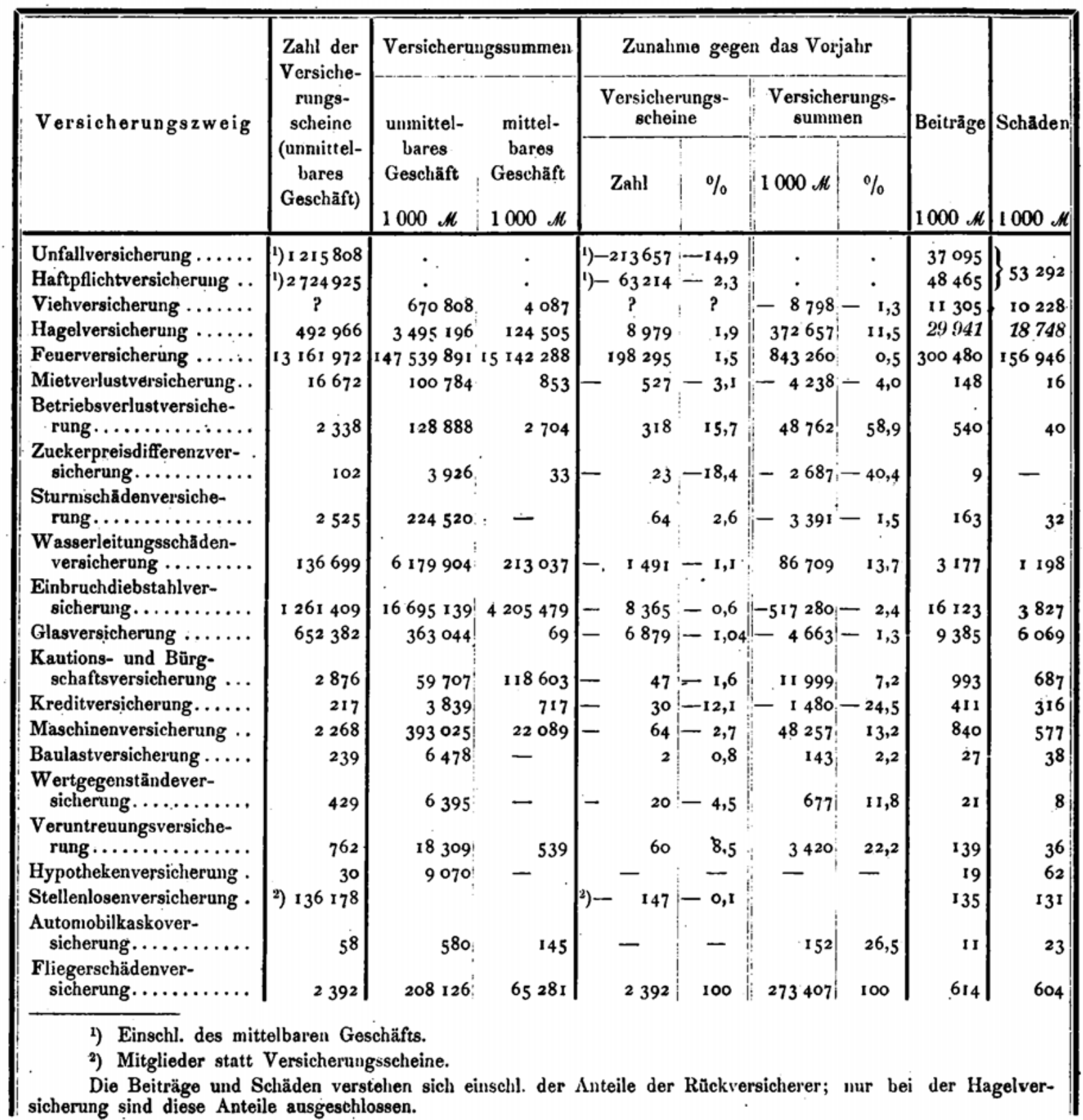


c) Versicherung in ausschlieBlich mittelbar betriebenen Zweigen

\begin{tabular}{|c|c|c|}
\hline Versicherungszweig & $\begin{array}{l}\text { Beitrảge } \\
\text { für eigene } \\
\text { Rechnung } \\
1000\end{array}$ & $\begin{array}{l}\text { Schäden } \\
\text { für eigene } \\
\text { Rechnung } \\
1000 \text { An }\end{array}$ \\
\hline Lebensversicherung . . . . . . . . & 48478 & 22444 \\
\hline Unfall- und Haftpflichtversicherung & 20737 & 9683 \\
\hline Hagelversicherung $\ldots \ldots \ldots \ldots \ldots$ & 6327 & 4423 \\
\hline Feuerversicherung $\ldots \ldots \ldots \ldots \ldots$ & 169368 & 96880 \\
\hline Sonstige aufsichtspflichtige $\mathbf{Z w e i g e .}$ & II 3 II & $404, I$ \\
\hline Zusammen $\ldots$ & 256221 & 137471 \\
\hline
\end{tabular}

Beurteilt man in der Unfall- und Haftpflichtversicherung den Umfang des Geschäfts nach der Jahresbeitragseinnahme, so kommen von dem mit 85,56 $(107,32)$ Millionen Mark zu bewertenden gesamten Geschäfte der deutschen Gesellschaften $78,37(98,54)$ Millionen Mark auf das deutsche und $7,19,(8,78)$ Millionen Mark auf das ausländische Geschäft; die ausländischen Gesellschaften haben aus ihrem deutschen Geschäfte 12,96 $(18,48)$ Millionen Mark eingenommen; sie sind also an dem gesamten deutschen Geschäfte mit $14,2(15,8)$ v. H. beteiligt gewesen.

Im unmittelbaren Feuerversicherungsgeschäfte hatten die großen deutschen Versicherungsgesellschaften insgesamt 147540 (141 956). Millionen Mark versichert; davon kamen 139013 (132 944) Millionen auf das deutsche und 8527 (9012) Millionen Mark auf das ausländische Geschäft. Die ausländischen Unternehmungen einschließlich der hier sonst nicht weiter berücksichtigten Unternehmungen mit weniger als $100000 \varkappa$ Beitragseinnahme aus dem deutschen Geschäfte hatten im unmittelbaren deutschen Geschäft um dieselbe Zeit 5740 (5 667) Millionen Mark übernommen. Von diesen Zahlen sind indessen die auf das ausländische Geschäft der deutschen Gesellschaften und die auf das deutsche Geschäft der ausländischen Gesellschaften bezüglichen unsieher; sie können nur als untere Grenze angesehen werden. Die deutschen Unternehmungen konnten keine vollständigen Angaben machen, weil ihnen der Verkehr mit ihren ausländischen Vertretungen infolge von Kriegsmaßnahmen erschwert oder unmöglich gemacht war und das Geschäft der Gesellschaften mit dem Sitze im feindlichen Auslande fehlt, soweit es nicht infolge der erwähnten Uberführungsverträge auf deutsche Gesellschaften übergegangen und bei diesen nachgewiesen ist. Rechnet man unter diesem Vorbehalte zu dem deutschen Geschäfte noch die Summen, welche nach den "Mitteilungen für die öffentlichen Feuerversicherungsanstalten " die im Deutschen Reiche bestehenden 48 öffentlichen Anstalten versichert hatten, nämlich 90896 (89530) Millionen Mark, so hat man in 235649 (228141) Millionen Mark sehr nahe den Mindestwert der gegen Feuersgefahr versicherten deutschen Objekte, an dem übrigens auch noch die bei den kleinen Vereinen und den unter Landesaufsicht stehenden privaten Gegenseitigkeitsverẹinen versicherten Werte fehlen, die insgesamt jedoch nur wenige Hundertteile der soeben angegebenen Summe ausmachen werden. - Von obiger Summe kamen auf die deutschen privaten Unternehmungen unter Reichsaufsicht 59,0 $(58,3)$ v. H., auf die ausländischen Gesellschaften 2,4 $(2,5)$ v. $H$. und auf die deutschen öffentlichen Anstalten 38,6 $(39,2)$ v. H.

Aus dem unmittelbaren deutschen Geschäfte sind von den obigen privaten Unterñehmungen und von den öffentlichen Feuerversicherungsanstalten im Jahre I9I5 für 
Lebensversicherung 692,06 (722,59), für Unfall- und Haftplichtversicherung 87,59 $(112,72)$ und für Feuerversicherung $338,89 \quad(317,96)$, insgesamt $1118,54 \quad(1153,27)$ Millionen Mark an Beiträgen vereinnahmt worden, das sind rund $3,1(3,2)$ Millionen Mark auf den Tag.

Die folgenden Ubersichten sollen einen Einblick in die Betriebsergebnisse des Berichtsjahrs und in den Vermögensstand am Schlusse des Geschäftsjahrs für die Gesamtheit der deutschen privaten Unternehmungen mit Ausnahme der Kaskoversicherungsvereine gewähren.

Hierzu ist folgendes zu bemerken: Es ist schon erwähnt, daß die meisten Aktiengesellschaften mehrere Versicherungszweige betreiben. Die Gesellschaften dieser Art geben dann hinsichtlich ihres Lebensversicherungsgeschäfts für die Volksversicherung einerseits und für die gewöhnliche Versicherung einschließlich der "sonstigen kleinen Versicherung " anderseits besondere Gewinn- und Verlustrechnungen, aus denen die folgenden Zahlen abgeleitet worden sind.

Hauptsächlichste Einnahme- und Ausgabeposten der Lebensversicherung $1000 \mu$

\begin{tabular}{|c|c|c|c|c|c|c|c|c|}
\hline Betriebsart & $\begin{array}{l}\text { Beitıäge } \\
\text { und } \\
\text { Ausferti- } \\
\text { gungs- } \\
\text { gebühren }\end{array}$ & $\begin{array}{l}\text { Ver- } \\
\text { gütungen } \\
\text { der } \\
\text { Rückver- } \\
\text { sicherer }\end{array}$ & $\begin{array}{c}\text { Kapital- } \\
\text { ertrãge } \\
\text { (rein) }\end{array}$ & $\begin{array}{c}\text { Zahlungen } \\
\text { für } \\
\text { Versiche- } \\
\text { rungsfälle }\end{array}$ & $\begin{array}{l}\text { Rück- } \\
\text { versiche- } \\
\text { rungs- } \\
\text { beiträge }\end{array}$ & $\begin{array}{c}\text { Zu- } \\
\text { führungen } \\
\text { zu den } \\
\text { Rücklagen } \\
\text { (rein) }\end{array}$ & $\begin{array}{c}\text { Ver- } \\
\text { waltungs- } \\
\text { kosten und } \\
\text { Steuern }\end{array}$ & $\begin{array}{c}\text { Jahres- } \\
\text { über- } \\
\text { schuß }\end{array}$ \\
\hline Volksversicherung*)....... & 118727 & I 838 & 30255 & 62132 & 1768 & 36606 & 26489 & 21828 \\
\hline $\begin{array}{l}\text { Große Versicherung und son- } \\
\text { stige kleine Versicherung* }\end{array}$ & 586142 & 29920 & 229640 & 459782 & 26218 & 159015 & 51764 & 147157 \\
\hline Berufsvereinigungen $\ldots \ldots$ & 16434 & 321 & 5729 & 5990 & 248 & 13255 & 708 & $\begin{array}{r}1528 \\
\end{array}$ \\
\hline Zusammen .... & 721303 & 32079 & 265624 & 527904 & 28234 & 208876 & 78961 & 170513 \\
\hline
\end{tabular}

*) Einschl. Zeitungsabonnentenversicherung.

Hinsichtlich der übrigen Versicherungszweige verfahren die Gesellschaften in ihren Rechnungsabschlüssen verschieden; sie trennen entweder alle Einnahme- und Ausgabeposten nach Geschäftszweigen oder nur einen Teil dieser Posten, während sie die übrigen, besonders die Kapitalerträge und die allgemeinen Verwaltungskosten, nur in einer Summe angeben. In den für das Aufsichtsamt bestimmten Aufstellungen werden indessen auch die allgemeinen Posten auf die einzelnen Zweige verteilt; daher kann in dieser Statistik für jeden Zweig der Gewinn aus dem eigentlichen Versicherungsbetrieb angegeben werden. Nur die Unfall- und Haftpflichtversicherung macht eine Ausnahme, weil hier von den Kapitalerträgen ein Teil dem Versicherungsbetriebe zugerechnet werden muß und dieser Teil nicht ausgeschieden werden kann. Aus den bezüglichen Tafeln ist die folgende Ubersicht zusammengestellt worden. Darin sind als Sonstige Betriebs-Einnahmen die Ersparnisse aus der Schadenrücklage sowie die Abnahme der Prämienreserven und Uberträge und bei der Viehwersicherung der Erlös aus verwertetem Vieh, als Sonstige Betriebs-Ausgaben etwaige Zuschüsse zur Schaden. rücklage und die Zunahme der Prämienreserven und Uberträge, bei der Feuerversiche. rung auch die Leistungen zu gemeinnützigen $Z$ wecken aufgeführt. Die Anteile der Rück. 
versicherer sind ausgeschlossen. Die Schadenzahlungen enthalten auch die Schadenermittelungskosten.

Die Rückversicherungsgesellschaften haben dem Aufsichtsamt über ihre Geschäftsführung in einer Form zu berichten, die eine Ermittelung des Gewinns aus dem reinen Versich rungsbetriebe weder für einzelne Zweige noch für das gesamte Geschäft gestattet; in die nachstehende Tafel sind daher als Sonstige Einnahmen und Ausgaben die Summen der Jahreseinnahmen (ohne Gewinnvortrag) und Jahresausgaben nach Abzug der Beiträge einerseits und der Schäden nebst Verwaltungskosten und Steuern anderseits aus der Gewinn- und Verlustrechnung eingestellt.

\section{Aus der Betriebsrechnung der deutschen Schaden- und Rückversicherungsunternehmungen für 1915}

\begin{tabular}{|c|c|c|c|c|c|c|}
\hline Versicherungszweig & $\begin{array}{c}\text { Betriebs-Ein } \\
\text { Beiträge } \\
\text { und Neben- } \\
\text { leistungen } \\
\text { der Ver- } \\
\text { sicherten }\end{array}$ & nnahmen & $\begin{array}{l}\text { Betriebs-A } \\
\text { Schaden- } \\
\text { zahlungen }\end{array}$ & Sonstige & $\begin{array}{c}\text { Ver- } \\
\text { waltungs- } \\
\text { kosten } \\
\text { und } \\
\text { Steuern }\end{array}$ & $\begin{array}{c}\text { Uberschuß } \\
\text { (Verlust - ) } \\
\text { des Ver- } \\
\text { sicherungs- } \\
\text { betriebs }\end{array}$ \\
\hline 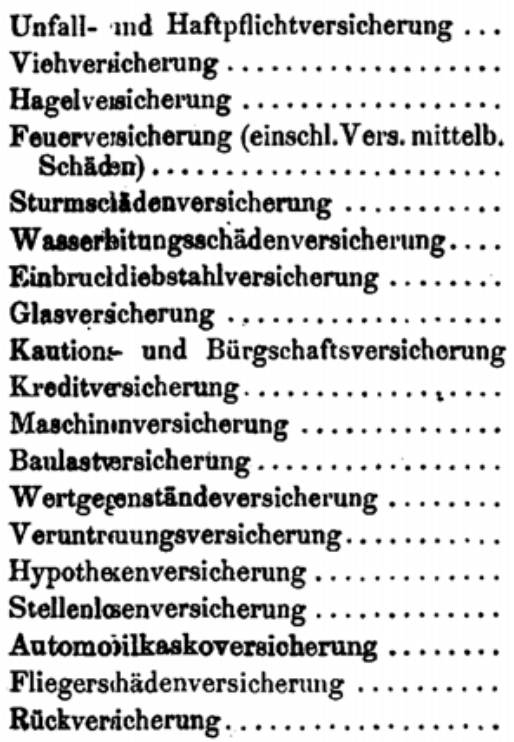 & \begin{tabular}{r|}
87171 \\
11040 \\
32463 \\
178042 \\
33 \\
2741 \\
11114 \\
8736 \\
603 \\
177 \\
294 \\
27 \\
10 \\
56 \\
8 \\
135 \\
6 \\
247 \\
310348
\end{tabular} & 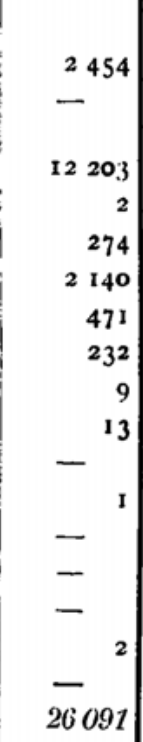 & \begin{tabular}{r|}
49408 \\
10079 \\
19782 \\
83486 \\
9 \\
1127 \\
4192 \\
5490 \\
706 \\
107 \\
183 \\
39 \\
4 \\
11 \\
7 \\
131 \\
13 \\
61 \\
171709
\end{tabular} & $\begin{array}{r}9575 \\
4 \\
44 \\
417 \\
124 \\
6 \\
20 \\
-\quad 9 \\
-\quad 1 \\
10 \\
-\quad 56 \\
-\quad \\
-\quad 175 \\
63322\end{array}$ & $\begin{array}{r}30089 \\
2412 \\
6055 \\
55730 \\
13 \\
957 \\
3914 \\
2904 \\
158 \\
46 \\
58 \\
2 \\
5 \\
22 \\
2 \\
50 \\
1 \\
76 \\
89701\end{array}$ & $\begin{array}{r}1003 \\
6626 \\
41454 \\
9 \\
887 \\
4731 \\
689 \\
35 \\
13 \\
57 \\
14 \\
1 \\
13 \\
57 \\
46 \\
6 \\
65 \\
12307\end{array}$ \\
\hline
\end{tabular}

Eine Bilanz wird immer nur für das Gesamtgeschäft aufgestellt und bei derjenigen Gruppe nachgewiesen, zu der die Gesellschaft ihrem aufsichtsplichtigen Hauptbetriebe nach zu rechnen ist. Die Bilanz umfaßt also gegebenenfalls auch das Transportversicherurgsgeschäft, über das die Statistik im übrigen nur bei den Rückversicherungsgesellschaften einige Angaben bringt, weil die Transportversicherung der Beaufsichtigung n.cht unterliegt. In den Gewinn der Bilanz ist auch der Gewinnvortrag aus dem Vocjahr eingeschlossen.

In der folgenden Ubersicht sind berücksichtigt 115 Aktiengesellschaften mit 653,00 Millionen Mark Aktienkapital, wovon 169,87 Millionen Mark eingezahlt sind, und 95 Gegenseitigkeitsvereine mit einem Garantiekapitale von 3,15 Millionen mit 1,73 Millionen 
Einzahlung. Die für die Aktiengesellschaften in $§ 262$ des Handelsgesetzbuchs und für die Gegenseitigkeitsvereine in $§ 37$ des Versicherungsaufsichtsgesetzes vorgeschriebenen gesetzlichen Rücklagen beliefen sich Ende 19 I 5 für alle Unternehmungen zusammen auf 194,44 Millionen; dazu kamen 353,10 Millionen Mark Sonderrücklagen, die freilich zum Teil nicht als freie Rücklagen betrachtet werden können. Die Prämienreserven und die Prämienüberträge, 5 801,17 Millionen, meist auch die Gewinnrücklagen, 591,35 Millionen, sowie die Schadenrücklagen, 356,04 Millionen, sind als gebunden anzusehen, da sie als rechnungsmäßige Deckung für die teils bereits entstandenen, teils voraussichtlich in Zụkunft noch entstehenden Ansprüche der Versicherten aus geleisteten Beitragszahlungen dienen.

Aus der Bilanz der deutschen Unternehmungen für Ende 1915 - Gesamtgeschäft

$1000 \mu$

\begin{tabular}{|c|c|c|c|c|c|c|c|}
\hline \multirow[b]{2}{*}{$\begin{array}{c}\text { Posten der Vermögenswerte } \\
\text { und Verbindlichkeiten }\end{array}$} & \multicolumn{7}{|c|}{ Hauptzweig } \\
\hline & $\begin{array}{c}\text { Lebens- } \\
\text { versiche- } \\
\text { rung }\end{array}$ & $\begin{array}{c}\text { Unfall- } \\
\text { und Haft- } \\
\text { pflicht- } \\
\text { versiche- } \\
\text { rung }\end{array}$ & $\begin{array}{l}\text { Vieh- } \\
\text { versiche- } \\
\text { rung }\end{array}$ & $\begin{array}{l}\text { Hagel- } \\
\text { versiche- } \\
\text { rung }\end{array}$ & $\begin{array}{c}\text { Feuer- } \\
\text { versiche- } \\
\text { rung }\end{array}$ & $\begin{array}{c}\text { Glas- } \\
\text { versiche- } \\
\text { rung }\end{array}$ & $\begin{array}{c}\text { Rūck- } \\
\text { versiche- } \\
\text { rung }\end{array}$ \\
\hline Vermögenswerte insgesamt.......... & 6769191 & 386536 & 9711 & 61041 & 813506 & 20009 & 595866 \\
\hline $\begin{array}{c}\text { Verpflichtungen der Aktionäre oder } \\
\text { Garanten } \ldots \ldots \ldots \ldots \ldots \ldots \ldots \ldots \ldots\end{array}$ & 120655 & 46154 & 750 & 19621 & 177631 & 4363 & I15 375 \\
\hline 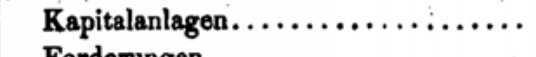 & 6285198 & 274395 & 5948 & 32861 & 508504 & 12818 & 249562 \\
\hline $\begin{array}{l}\text { Forderungen } \ldots \ldots \ldots \ldots \ldots \ldots \ldots \\
\text { Kassenbestand } \ldots \ldots \ldots \ldots \ldots \ldots \ldots \ldots\end{array}$ & $\begin{array}{r}307318 \\
6487\end{array}$ & $\begin{array}{r}62201 \\
450\end{array}$ & $\begin{array}{r}2748 \\
.154\end{array}$ & $\begin{array}{r}8343 \\
212\end{array}$ & $\begin{array}{r}122344 \\
125^{8}\end{array}$ & $\begin{array}{r}2490 \\
54\end{array}$ & $\begin{array}{r}187622 \\
173\end{array}$ \\
\hline Ubrige Vermögenswerte .......... & 49533 & 3336 & 111 & 4 & 3769 & 284 & 43134 \\
\hline Verlust.$\ldots \ldots \ldots \ldots \ldots \ldots \ldots \ldots \ldots$ & - & - & - & - & - & - & - \\
\hline Verbindliohkeiten insgesamt $\ldots \ldots \ldots \ldots$ & 6769191 & 386536 & 9711 & 61041 & 813506 & 20009 & 595866 \\
\hline Aktien- oder Garantiekapital . . . . . . & 163483 & 62000 & I 000 & 25529 & 242547 & 6312 & 155286 \\
\hline Gesetzliche Rücklage $\ldots \ldots \ldots \ldots \ldots$ & 33215 & 34050 & 4233 & 12149 & 71039 & 1672 & 38084 \\
\hline $\begin{array}{l}\text { Sonderrücklagen } \ldots \ldots \ldots \ldots \ldots \ldots \ldots \\
\text { Gewinnrücklagen } \ldots \ldots \ldots \ldots \ldots \ldots \ldots\end{array}$ & 194140 & 37394 & 248 & ${ }^{13}{ }^{178}$ & 73124 & $-^{925}$ & $\begin{array}{l}34093 \\
-\end{array}$ \\
\hline $\begin{array}{c}\text { Guthaben von Versicherungsunter- } \\
\text { nehmungen........................ }\end{array}$ & 18446 & 21996 & 72 & 4 & 46247 & II5 & 51890 \\
\hline Prämienreserven u. Prämienübertrāge & 5304640 & II 9623 & I 743 & 1 & $2095^{8} 5$ & 5210 & 160364 \\
\hline Schadenrücklagen $\ldots \ldots \ldots \ldots \ldots \ldots$ & 80140 & 67331 & 540 & 768 & 73663 & 3433 & 130165 \\
\hline Ubrige Verbindlichkeiten .......... & 222322 & 13701 & 1368 & 3099 & 42307 & I 316 & 8246 \\
\hline Gewinn..$\ldots \ldots \ldots \ldots \ldots \ldots \ldots \ldots$ & 170501 & 21398 & 507 & 6313 & 54994 & 1026 & 17738 \\
\hline
\end{tabular}

Die Kapitalanlagen aller dieser 207 Unternehmungen haben Ende I 915 insgesamt 7 369,29 Millionen Mark betragen; davon entfallen 183,12 Millionen auf Grundbesitz, 5 300,40 Millionen auf Hypotheken und Grundschulden, 345,89 Millionen auf Darlehen an öffentliche Körperschaften, 9,12 Millionen auf Darlehen auf Wertpapiere, 991,19 Millionen auf Wertpapiere, 530,60 Millionen auf Darlehen auf Versicherungen, 4,49 Millionen auf Wechsel und 4,48 Millionen auf sonstige Anlagen. 
Die Verteilung auf diese Wertarten ist aber bei den verschiedenen Versicherungszweigen und zum Teil auch bei den beiden Geschäftsformen eine recht verschiedene, auch wird sie durch heimische Ubung und Gesetzgebung mannigfach beeinflußt. Năheres hierüber findet man in der "Entwickelung “ S. 62*).

\section{Kapitalanlagen Ende 1915 (Bilanzwert)}

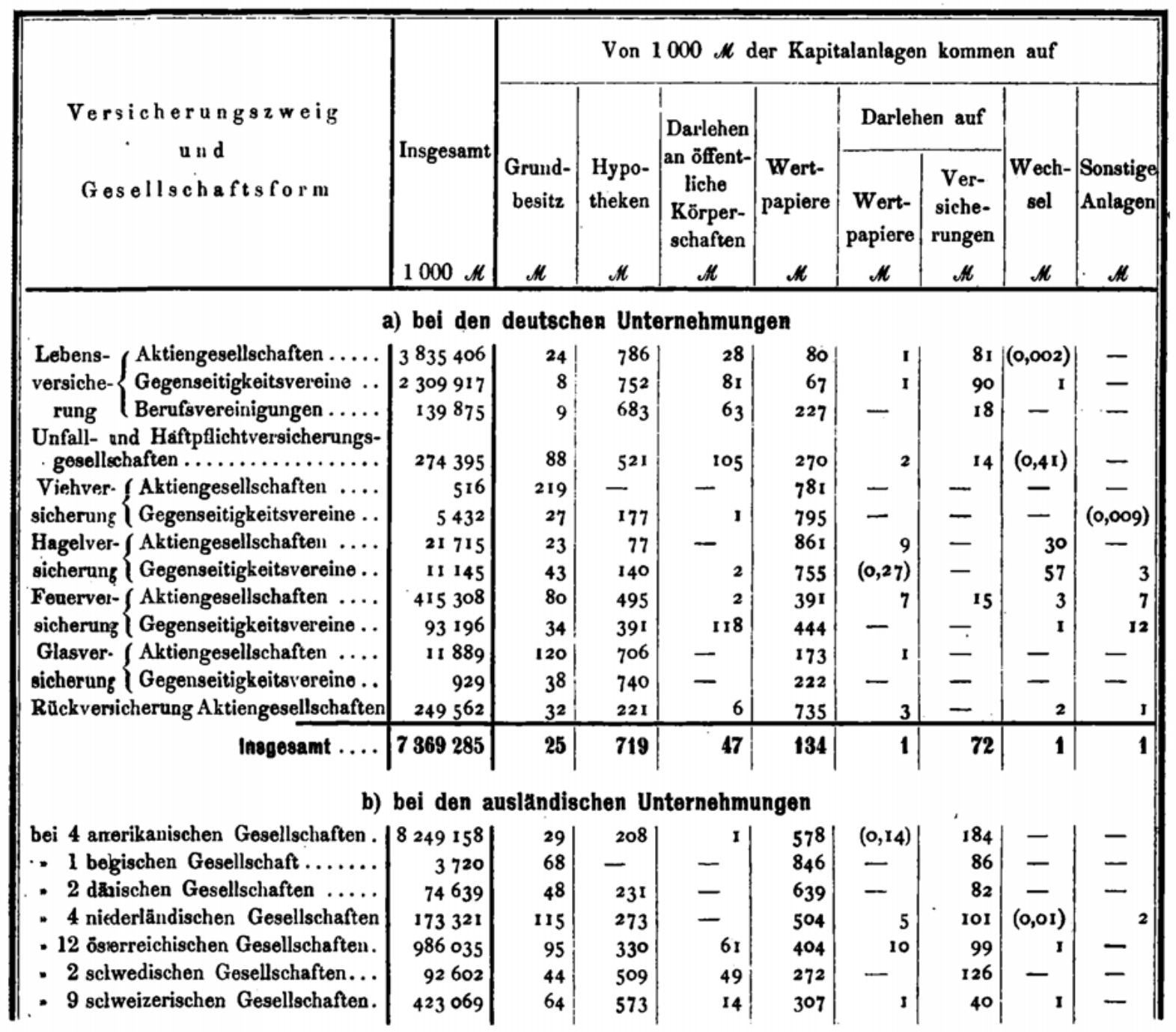

Uber die Zusammensetzung der unter den Kapitalanlagen aufgeführten Wertpapierbestände soll die folgende Zusammenstellung Aufschluß geben. Zu bemerken ist dazu nur, daß der Verteilung der Nennwert zugrunde gelegt worden ist und daß der bej den einzelnen Zweigen eingestellte Nennwert des Gesamtbestandes wegen besonderer Umstände, die aus den Fußnoten zu den betreffenden Tafeln ersichtlich sind und hier nicht angeführt zu werden brauchen, nicht überall genau dem bei den Kapitalenlagen in der Bilanz verrechneten Werte entspricht; die Abweichungen sind abrigen so gering, daß sie hier nicht ins Gewicht fällen.

*) Jie Entwickelung des privaten Versicherungswesens unter Reichsaufsicht in dem Jahrfünft 1907 bis 1911. Herausgeg’ben vom Kaiserlichen Aufsichtsamte für Privatversicherung, Berlin 1913, J. Guttentag, Verlagsbuchhandlung, G: m. b. H - Diese Schrift ist in dem vorliegenden Buche kurz als -Entwickelung* angeführt. 
Verteilung der Wertpapiere (Nennwert)

\begin{tabular}{|c|c|c|c|c|c|c|c|c|c|}
\hline \multirow{3}{*}{$\begin{array}{c}\text { Versicherungszweig } \\
\text { und } \\
\text { Gesellschaftsform }\end{array}$} & \multirow{3}{*}{$\begin{array}{c}\text { Wert- } \\
\text { papiere } \\
\text { ins- } \\
\text { gesamt } \\
\\
1000 \AA\end{array}$} & \multicolumn{8}{|c|}{ Von $1000 \mathscr{A}$ der Wertpapiere kommen auf } \\
\hline & & \multicolumn{5}{|c|}{ inländische Wertpapiere } & \multicolumn{3}{|c|}{ ausländische Wertpapiere } \\
\hline & & $\begin{array}{c}\text { Staats- } \\
\text { anleihen } \\
\text { und } \\
\text { staatlich } \\
\text { gedeckte } \\
\text { Anleihen } \\
\mathscr{M}\end{array}$ & $\mid \begin{array}{c}\text { Kom- } \\
\text { munal- } \\
\text { anleihen } \\
\mathscr{N}\end{array}$ & $\begin{array}{c}\text { Pfand- } \\
\text { briefe } \\
\text { und } \\
\text { Kommu- } \\
\text { nalobli- } \\
\text { gationen } \\
\mathscr{M}\end{array}$ & \begin{tabular}{|c|} 
sonstige \\
Schuld- \\
ver- \\
schrei- \\
bungen \\
$\mu$
\end{tabular} & Aktien & $\begin{array}{c}\text { staat- } \\
\text { liche und } \\
\text { komnu- } \\
\text { nale } \\
\text { Anleihen } \\
\mathscr{M}\end{array}$ & \begin{tabular}{|c|} 
sonstige \\
Schuld- \\
ver- \\
schrei- \\
bungen \\
$\mu$
\end{tabular} & Aktien \\
\hline Lebens- (Aktiengesellschaften..... & 328286 & 687 & 12 & $4 \mathrm{I}$ & 1 & 12 & 244 & 2 & I \\
\hline versiche- $\{$ Gegenseitigkeitsvereine.. & 167322 & 805 & 55 & 45 & - & 5 & 87 & 3 & - \\
\hline rung ( Berufsvereinigungen...... & 33875 & 904 & 67 & 28 & - & - & $\mathbf{1}$ & & - \\
\hline $\begin{array}{l}\text { Unfall- und Haftpflichtversicherungs- } \\
\text { gesellschaften } \ldots \ldots \ldots \ldots \ldots \ldots \ldots \ldots\end{array}$ & 83202 & 638 & 45 & 22 & $(0,024)$ & 67 & $15^{8}$ & 68 & 2 \\
\hline Viehver- (Aktiengesellschaften .... & 431 & 678 & - & 193 & - & - & 129 & - & - \\
\hline sicherung Gegenseitigkeitsvereine .. & $474 \mathrm{I}$ & 572 & 195 & 225 & 6 & - & 2 & 一 & - \\
\hline Hagelver- $\{$ Aktiengesellschaften .... & 19497 & 817 & 5 & 32 & 3 & 131 & 12 & - & - \\
\hline sicherung $\{$ Gegenseitigkeitsvereine... & 9168 & 692 & 78 & 230 & - & - & - & - & - \\
\hline Feuerver- (Aktiengesellschaften $\ldots$. & I 84868 & $66 \mathrm{I}$ & 38 & 59 & 2 & & 126 & 90 & 9 \\
\hline sicherung / Gegenseitigkeitsvereine .. & 47093 & 632 & 188 & 152 & 3 & $(0,057)$ & 12 & 13 & - \\
\hline Glasver- ( Aktiengesellschaften .... & 2448 & 612 & 12 & 43 & - & $5^{8}$ & 273 & 2 & - \\
\hline sicherung / Gegenseitigkeitsvereine.. & 231 & 527 & 26 & 282 & $\mathrm{r}_{5}^{8}$ & - & 7 & - & - \\
\hline Rückversicherung Aktiengesellschaften & 222206 & 362 & 42 & 46 & 13 & 107 & 182 & 135 & 113 \\
\hline Insgesamt .... & 1103368 & 637 & 42 & 51 & 3 & 36 & 157 & 49 & 25 \\
\hline
\end{tabular}

In der obigen Bilanz-Tafel sind bei jedem Versicherungszweige die Verluste, di einige Unternehmungen zu verzeichnen hatten, gegen die Gewinne der anderen Gesell schaften verrechnet. Es ergibt sich so für die Gesamtheit der deutschen Versicherungs unternehmungen ein Rein-Gewinn von 272,477 Millionen Mark. Handelt es sich aber un die Verteilung des Gewinns, so kommt der durch die Bilanz ausgewiesene Gewinn alle derjenigen Gesellschaften in Betracht, die mit finanziell günstigem Erfolge gearbeite haben, das sind 273,308 Millionen Mark. Wie dieser Betrag verteilt worden ist, zeig folgende Ubersicht:

\begin{tabular}{|c|c|c|c|c|c|c|c|c|}
\hline $\begin{array}{l}\text { Von dem Gewinne sind in } 1000 \varkappa \\
\text { abgeführt worden }\end{array}$ & \begin{tabular}{|} 
an die \\
gesetz- \\
liche \\
Rücklage
\end{tabular} & $\begin{array}{c}\text { an } \\
\text { sonstige } \\
\text { Rück- } \\
\text { lagen }\end{array}$ & $\begin{array}{c}\text { an den } \\
\text { Beamten- } \\
\text { wohl- } \\
\text { fahrts- } \\
\text { stock }\end{array}$ & $\begin{array}{c}\text { an die } \\
\text { Aktionäre } \\
\text { oder } \\
\text { Garanten }\end{array}$ & $\begin{array}{c}\text { an Tan- } \\
\text { tieme- } \\
\text { be- } \\
\text { rechtigte }\end{array}$ & $\begin{array}{c}\text { an die } \\
\text { Ver- } \\
\text { sicherten }\end{array}$ & $\begin{array}{l}\text { ander- } \\
\text { weit }\end{array}$ & $\begin{array}{l}\text { Vortrag } \\
\text { auf neue } \\
\text { Rechnung }\end{array}$ \\
\hline Lebensve & 746 & 14326 & 753. & 7783 & 2781 & 145337 & I 621 & I 237 \\
\hline Unfall- und Haftpflichtversicherung. & 7 & 685 & 412 & 5987 & 1575 & 5917 & I 296 & 2258 \\
\hline Viehversicherung $\ldots \ldots \ldots \ldots \ldots$. & 92 & 238 & 9 & 25 & 91 & 18 & 18 & 18 \\
\hline Hagelversicherung...... & 628 & I 806 & $150^{-1}$ & I 899 & 423 & II 8 & 1083 & 206 \\
\hline Feuerversicherung usw.......... & I 537 & 5684 & I 042 & 14813 & 2404 & 23670 & I 924 & 3926 \\
\hline Glasversicherung $\ldots \ldots \ldots \ldots \ldots$ & $3^{8}$ & 300 & - & 375 & 182 & 30 & 40 & 62 \\
\hline Rückversicherung $\ldots . .$. & 462 & 1022 & 90 & 8215 & 880 & - & 673 & 6396 \\
\hline Zusammen .... & 3510 & 24061 & 2456 & 39097 & 8336 & 175090 & 6655 & 14103 \\
\hline
\end{tabular}


Außerdem sind den Versicherten der Lebensversicherungsabteilungen verschiedener Aktiengesellschaften zusammen noch 5,769 Millionen vor dem Abschluß der Jahresrechnung als Gewinnanteil überwiesen worden, die in vorstehender Ubbersicht nicht verrechnet sind.

Schließlich mag hier noch eine Gegenüberstellung der Beiträge und der Schäden der unmittelbar arbeitenden Unternehmungen, beide einschließlich der Anteile der Rückversicherer und des mittelbaren Geschäfts, bei den verschiedenen Versicherungszweigen Platz finden, die einerseits das ausländische Geschäft der deutschen Gesellschaften und anderseits das deutsche Geschäft der ausländischen Gesellschaften betreffen. Es sei aber besonders hervorgehoben, daß aus den Endzahlen keineswegs Schlüsse auf die Verhältnisse des Versicherungsgeschäfts überhaupt gezogen werden dürfen; dazu fehlen die Zahlen für die ganz vornehmlich internationalen Betriebe der Transportversicherung und

Das anslandische Geschsin der deutschen und das dentsoho Geschant der ausIsndisahen Gesellschaften der Rückversicherung. Auch ist zu beachten, daß die Zahlen für I9I5 auch bei den deutschen Gesellschaften nicht vollständig sind, weil die Gesellschaften über ihr Geschäft im feindlichen Auslande nicht immer hinreichend unterrichtet sind, und daß bei den ausländischen Gesellschaften die englischen und die französischen in diesem Jahre wiederum fehlen.

\begin{tabular}{|c|c|c|c|c|}
\hline \multirow[b]{2}{*}{ Versicherungszweig } & \multicolumn{2}{|c|}{$\begin{array}{l}\text { Ausländisches Geschäft der. } \\
\text { deutschen Gesellschaften }\end{array}$} & \multicolumn{2}{|c|}{$\begin{array}{l}\text { Deutsches Geschäft der aus- } \\
\text { ländischen Gesellschaften }\end{array}$} \\
\hline & $\begin{array}{l}\text { Beiträge } \\
1000 \mathscr{H}\end{array}$ & $\begin{array}{l}\text { Schäden } \\
1000 \mu\end{array}$ & $\begin{array}{l}\text { Beiträge } \\
1000 \mathscr{N}\end{array}$ & $\begin{array}{l}\text { Schäden } \\
1000 \bumpeq \mathbb{N}\end{array}$ \\
\hline 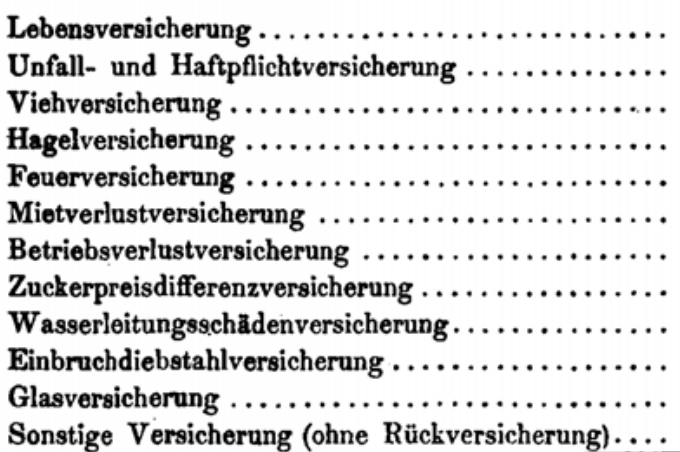 & $\begin{array}{r}67632 \\
7188 \\
155 \\
226 \\
63527 \\
3 \\
12 \\
-\quad \\
111 \\
1687 \\
707 \\
281\end{array}$ & $\begin{array}{rr}34 & 638 \\
5552 \\
128 \\
136 \\
38686 \\
-\quad \\
-\quad \\
-\quad \\
\\
\\
\\
\\
\quad 33 \\
571 \\
433 \\
111\end{array}$ & \begin{tabular}{rr}
45424 \\
12964 \\
$-\quad$ \\
$-\quad$ \\
\multicolumn{1}{r}{10595} \\
3 \\
$-\quad 8$ \\
\\
\\
\\
\\
12 \\
925 \\
21 \\
161
\end{tabular} & $\begin{array}{r}39350 \\
6036 \\
-\quad \\
-\quad 5825 \\
\\
1 \\
-\quad 32 \\
\\
\\
271 \\
10 \\
159\end{array}$ \\
\hline 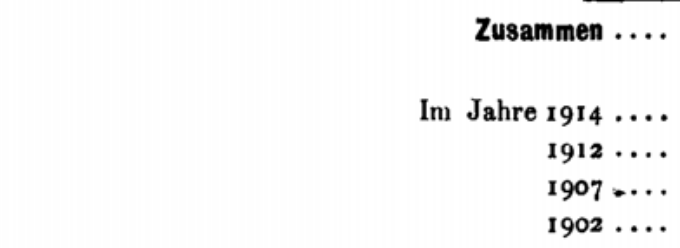 & $\begin{array}{r}141529 \\
162840 \\
160064 \\
109753 \\
90930\end{array}$ & $\begin{array}{ll}80 & 288 \\
94 & 566 \\
90 & 130 \\
60002 \\
54 & 163\end{array}$ & \begin{tabular}{ll|}
70 & 113 \\
77 & 476 \\
98 & 375 \\
81 & 942 \\
61 & 383
\end{tabular} & $\begin{array}{l}51688 \\
\\
55459 \\
62942 \\
55344 \\
33219\end{array}$ \\
\hline
\end{tabular}




\section{Gruppe I, Lebens- und Krankenversicherung}

Die Unternehmungen
Im Jahre 1915 standen auf dem Gebiete der Lebensversicherung und der Krankenversicherung 30 deutsche Aktiengesellschaften und eine große Zahl deutscher Gegenseitigkeitsvereine unter Reichsaufsicht.

Während 30 Aktiengesellschaften und 16 große Gegenseitigkeitsvereine ihren Geschäftsbetrieb für alle Kreise der Bevölkerung offen halten, versichert die Mehrzahl der Gegenseitigkeitsvereine nur die Angehörigen mehr oder weniger eng begrenzter Berufe. Von diesen Vereinen hat nur. einer (der Preußische Beamtenverein in Hannover) einen so großen Versicherungsbestand gewonnen, daß man ihn den anderen Gegenseitigkeitsvereinen, die eine Beschränkung auf bestimmte Berufe nicht kennen, an die Seite stellen kann; deshalb ist er auch hier jenen allgemeinen Vereinen zugesellt worden. Von den anderen Gegenseitigkeitsvereinen für beschränkte Personengruppen sind in die Tafeln dieser Statistik nur einige der größeren Versicherungseinrichtungen von Berufsvereinigungen aufgenommen. Die große Mehrzahl von Pensions- und Sterbekassen, die vielfach nur die Versicherung der Angestellten bestimmter gewerblicher Firmen bezwecken, sind wie in den früheren Jahren aus den Tafeln ganz fortgelassen; sie werden weiter unten nur im 'Text und auch da nur ganz zusammenfassend behandelt.

Die größeren Pensions- und Sterbekassen gehören ihrem ganzen Betriebe nach eher zur kleinen als zur großen Versicherung; sie sind hier aber gesondert be- ' handelt und als Versicherungseinrichtungen von Berufsvereinigungen zusammengefaßt. Die Bezeichnung "kleine Versicherung " ist im folgenden lediglich für die kleine Versicherung der im allgemeinen allen Bevölkerungsklassen zugänglichen großen Unternehmungen verwendet.

Von allen dem Aufsichtsamt unterstehenden deutschen Lebensversicherungsvereinen auf Gegenseitigkeit sind in den Tafeln dieser Statistik 18 größere Gegenseitigkeitsvereine allgemeinen Charakters und 14 Versicherungseinrichtungen von Berufsvereinigungen behandelt.

Eine Aktiengesellschaft - Nordstern, Unfall- und Haftpflicht-Versicherungs-AktienGesellschaft in Berlin-Schöneberg - hat sich, soweit Versicherungen der Gruppe I in Frage kommen, nur an der kleinen Versicherung beteiligt.

Bei den Gegenseitigkeitsvereinen ist die Mittelstandsversicherung a. G. Privater Versicherungsverein auf Gegenseitigkeit in Hamburg hinzugekommen, sie betreibt nur die Volksversicherung. Dagegen ist die Deutsche Militärdienst- und Lebens-VersicherungsAnstalt auf Gegenseitigkeit in Hannover durch Fusion auf die Berlinische Lebens-Versicherungs-Gesellschaft in Berlin übergegangen und erscheint deshalb in dieser Statistik nicht mehr unter besonderer Firma.

Bei den Berufsvereinigungen ist die Hinterbliebenen- und Altersversorgungs-Kasse des Vereins für Handlungs-Commis von $185^{8}$ (Kaufmännischer Verein) in Hamburg, 
Versicherungsverein auf Gegenseitigkeit, auf die Pensions-Kasse desselben Vereins übertragen worden, die fortab den Namen "Deutsche Welt « Lebensversicherungsgesellschaft a. G. des Kaufmännischen Vereins von I 858 , Hamburg, führt.

Von ausländischen Lebensversicherungsgesellschaften sind im Jahre I9I5 nur 17 in der Statistik behandelt, da 2 englische und 2 französische Gesellschaften infolge des Krieges keine Geschäftsberichte eingereicht haben. Da ein Geschäftsbericht der Assicurazioni Generali in Triest für I9 5 nicht zu erlangen war, sind in den Tafeln dieser Statistik bei dieser Gesellschaft durchweg die vorjährigen Zahlen eingestellt worden.

Von den 30 deutschen Aktiengesellschaften betrieben im Jahre 1915 neben der Lebensversicherung 15 auch die Unfallversicherung, 15 die Haftpflichtversicherung, 4 die Feuerversicherung, 3 die Kautionsversicherung (in ganz bescheidenem Umfang), 4 die Transportversicherung und 4 die Versicherung gegen Einbruchdiebstahl. Von den Gegenseitigkeitsvereinen hat nur einer (der Allgemeine Deutsche Versicherungs-Verein in Stuttgart) außer in der Lebensversicherung noch in der Unfall- und Haftpflichtversicherung und in einigen kleinen Nebenzweigen Geschäfte gemacht.

Von den allen Kreisen der Bevölkerung zugänglichen deutschen Unternehmungen unter Reichsaufsicht sind im Jahre I9I5 tätig gewesen

in der großen Versicherung

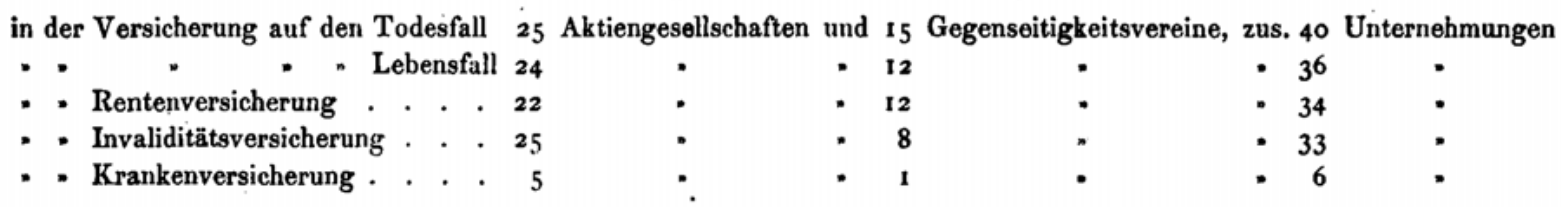

in der kleinen Versicherung

in der Volksversicherung. . . 12 Aktiengesellschaften und 4 Gegenseitigkeitsvereine, zusammen 16 Unternehmungen

- Zeitungsabonnentenvers. . 2

- sonstigen kleinen Vers. . I5

Von den 17 ausländischen Gesellschaften, über welche diese Statistik berichtet, haben im Deutschen Reiche gearbeitet sämtliche in der großen Versicherung auf den Todesfall, 16 in der Versicherung auf den Lebensfall, 16 in der Rentenversicherung, 6 in der Invaliditätsversicherung (Zusatzversicherung) und 4 in der Volksversicherung.

Vom Standpunkte der Statistik ist eine möglichst vollständige 'Trennung des großen Geschäfts von dem kleinen Geschäfte wünschenswert; denn der Betrieb ist verschieden, und die Versicherungen dienen zum Teil verschiedenen Zwecken.

Nun haben nach den Rechnungsvorschriften diejenigen Versicherungsunternehmungen, deren Geschäftsbetrieb die sogenannte kleine Lebensversicherung (Volksversicherung, Arbeiter-, Sterbekassen- usw. Versicherung) ohne ärztliche Untersuchung oder mit unvollständiger ärztlicher Untersuchung in der Art umfaßt, daß für diesen Versicherungsbetrieb besondere geschäftliche Einrichtungen und besondere, von den sonstigen Geschäftsgrundsätzen der Unternehmung wesentlich abweichende allgemeine Versicherungsbedingungen, Tarife und Gebührensätze eingeführt sind, für diesen Versicherungsbetrieb besondere Nachweisungen, namentlich auch über den Versicherungsbestand und seine Bewegung sowie hinsichtlich der Gewinn- und Verlustrechnung, auf- 
zustellen. Sofern aber die Unternehmungen für diesen Versicherungsbetrieb keine besonderen Einrichtungen haben, sind sie berechtigt, die kleine Versicherung mit der großen zusammen nachzuweisen.

Unter diesen Umständen ist es nicht möglich gewesen, die kleine Versicherung vollständig aus dem gesamten Geschäfte herauszuschälen. Bei einigen Unternehmungen konnte die Trennung nur beim Versicherungsbestande, nicht aber auch bei der Bewegung des Bestandes vollständig durchgeführt werden. Wo die Scheidung mit Hilfe der dem Aufsichtsamt eingereichten Ubersichten oder der gedruckten Geschäftsberichte möglich war, ist sie vorgenommen worden, obwohl dadurch eine gewisse Ungleichmäßigkeit zwischen den einzelnen Abschnitten dieser Statistik entstehen mußte; es erschien besser, diese Ungleichmäßigkeit in den Kauf zu nehmen, als auf eine strenge Scheidung der beiden Betriebsarten vollständig zu verzichten. Da, wo die Unternehmungen für die kleine Versicherung besondere geschäftliche Einrichtungen obengenannter Art geschaffen haben und wo mithin das kleine Geschäft von dem großen vollständig getrennt gehalten werden kann, wird in dieser Statistik die kleine Versicherung als "Volksversicherung ", wo das nicht der Fall ist, als "sonstige kleine Versicherung “ bezeichnet.

Der Beobachtungsstoff ist wie bisher folgendermaßen angeordnet.

Es ist zunächst der Versicherungsbestand am Ende des Berichtsjahres behandelt (Tafeln I 1 bis I 18); es folgt die Bewegung des Versicherungsbestandes (Tafeln I 19 bis I 28), dann die Gewinn- und Verlustrechnung (Tafeln I 29 bis I 36) und endlich die Bilanz für den Jahresschluß (Tafeln I 37 bis I 48).

In den einzelnen Abschnitten werden nacheinander berücksichtigt: die großen deutschen Unternehmungen (Aktiengesellschaften und Gegenseitigkeitsvereine), zuerst mit ihrer großen, dann mit ihrer kleinen Versicherung, danach die Versicherungseinrichtungen einiger größerer deutscher Berufsvereinigungen (Pensionskassen und Sterbekassen) und endlich die ausländischen Unternehmungen. Dabei wird von den deutschen Unternelımungen in den ersten drei Abschnitten immer das Lebensversicherungsgeschäft (inländisches und ausländisches) allein betrachtet, während die Bilanz für das Gesamtgeschäft (Lebensversicherung nebst allen Nebenzweigen) gegeben wird. Von den ausländischen Unternehmungen wird der Bestand und die Bewegung nur für das deutsche Geschäft gegeben; dagegen bezieht sich die Betriebsrechnung auf das ganze Lebensversicherungsgeschäft und die Bilanz auf das Gesamtgeschäft.

Hieran schließt sich eine Vergleichung des ausländischen Geschäfts der deutschen Unternehmungen mit dem deutschen Geschäfte der ausländischen Gesellschaften in den wichtigsten Punkten; endlich folgen einige Angaben über die dem Aufsichtsamt unterstehenden kleinen Pensions- und Sterbekassen.

\section{Der Versicherungsbestand am Ende des Jahres 1915}

Deutsche allgemeine

Über den gesamten Bestand der unter Reichsaufsicht stehenden deutschen Lebensversicherungsunternehmungen, die dem großen Publikum dienen, geben die Tafeln I 1 bis I 10 ausführlichen Aufschluß; hier sollen nur die hauptsächlichsten, für die Gesamtheit der Anstalten geltenden Ergebnisse mitgeteilt werden. 
In der folgenden Zusammenstellung sind zunächst die Summen der von den einzelnen Unternehmungen Ende I 9 I 5 überhaupt übernommenen Versicherungen wiedergegeben; diese Summen enthalten also alle Beträge doppelt, die von einer Gesellschaft, welche in unserer Statistik behandelt wird, versichert und bei einer ebensolchen Gesellschaft in Rückdeckung gegeben sind. Daneben ist angegeben, wieviel von je $1000 \mathscr{N}$ überhaupt übernommener Versicherung durchschnittlich für eigene Rechnung behalten worden ist.

\begin{tabular}{|c|c|c|c|c|c|c|}
\hline \multirow{2}{*}{ Versicherungsart } & \multicolumn{3}{|c|}{$\begin{array}{l}\text { Uberhaupt übernommene Versicherungen } \\
\text { in } 1000 \mathscr{H}\end{array}$} & \multicolumn{3}{|c|}{$\begin{array}{l}\text { Von } 1000 \mathscr{H} \text { überhaupt über- } \\
\text { nommener Vers. waren für } \\
\text { eigene Rechnung behalten }\end{array}$} \\
\hline & $\begin{array}{l}\text { Aktien- } \\
\text { gesell- } \\
\text { schaften }\end{array}$ & $\begin{array}{l}\text { Gegen- } \\
\text { seitigkeits- } \\
\text { vereine }\end{array}$ & zusammen & $\begin{array}{l}\text { Aktien- } \\
\text { gesell- } \\
\text { schaften }\end{array}$ & $\begin{array}{c}\text { Gegen- } \\
\text { seitigkeits- } \\
\text { vereine }\end{array}$ & zusammen \\
\hline Große Versicherung & & & & & & \\
\hline $\begin{array}{c}\text { Versicherung auf den Todesfall......... } \\
,\end{array}$ & $\begin{array}{r}7617747 \\
493739\end{array}$ & $\begin{array}{r}5651328 \\
146686\end{array}$ & $\begin{array}{r}13269075 \\
640425\end{array}$ & $\begin{array}{l}921 \\
980\end{array}$ & $\begin{array}{r}988 \\
\mathrm{I} 000\end{array}$ & $\begin{array}{l}95^{\circ} \\
985\end{array}$ \\
\hline $\begin{array}{l}\text { Rentenversicherung. } \ldots \ldots \ldots \ldots \ldots \ldots \\
\qquad \mathrm{K} \text { leine Versicherung }\end{array}$ & 21943 & 5546 & 27489 & 942 & 999 & 953 \\
\hline $\begin{array}{c}\text { Volksversicherung auf den Todesfall .. } \\
\text { Sonstige kleine Versicherung } \ldots \ldots \ldots\end{array}$ & $\begin{array}{r}1444454 \\
65^{2} \\
56494\end{array}$ & $\begin{array}{l}91352 \\
- \\
153224\end{array}$ & $\begin{array}{r}\text { I } 535806 \\
652 \\
2097 \text { r } 8\end{array}$ & $\begin{array}{l}982 \\
667 \\
994\end{array}$ & $\begin{array}{c}1000 \\
- \\
973\end{array}$ & $\begin{array}{r}983 \\
667 \\
979\end{array}$ \\
\hline
\end{tabular}

Die Rückversicherung spielt hiernach in der Lebensversicherung im ganzen nur eine bescheidene Rolle, zumal bei den Gegenseitigkeitsvereinen, bei denen sie fast nur in der großen Kapitalversicherung auf den Todesfall in Betracht kommt. Bei der Lebensfall- und der Rentenversicherung, sowie bei der kleinen Versicherung tritt sie eigentlich nur da auf, wo Gesellschaften vertragsmäßig verpflichtet sind, von jeder von ihnen abgeschlossenen Versicherung einen bestimmten Hundertsatz in Rückdeckung zu geben (sogenannte Quotenrückversicherung).

Fragt man dann weiter, wie sich der Versicherungsbestand der deutschen Gesellschaften aus selbst abgeschlossenen und aus in Rückdeckung übernommenen Versicherungen zusammensetzt, so ersieht man aus den Tafeln I 3 bis I 7 und I 9 und 10, daß die Gegenseitigkeitsvereine Ende I9 5 nur wenige Versicherungen auf den Todesfall und vereinzelte Jahresrenten in Rückdeckung genommen und daß auch die Aktiengesellschaften im großen und ganzen nur wenig mittelbare Geschäfte abgeschlossen haben.

In der großen Todesfallversicherung haben die Unternehmungen noch nicht einmal halb so viel in Rückdeckung übernommen, wie sie in Rückdeckung gegeben haben; bei den anderen großen Versicherungen tritt die Rückversicherung ganz zurück.

In der Volksversicherung ist das mittelbare Geschäft kaum nennenswert.

Besondere Beachtung verdienen die Zahlen, die sich auf die unmittelbaren, selbst abgeschlossenen Versicherungen beziehen, weil darin ein Maßstab für den Versicherungsbedarf der Bevölkerung gefunden werden kann. Wir geben davon sowohl die Zahl der Versicherungsscheine als auch die versicherten Summen und die durchschnittlich auf einen Schein entfallenden Beträge und setzen zum Vergleiche die Zahlen für I9 Io daneben. 
Unmittelbares Geschäft der deutschen allgemeinen Unternehmungen

Versicherungssummen in $1000 \mathscr{M}$, Jahresrenten und Durchschnittsbeträge in $\mathscr{N}$

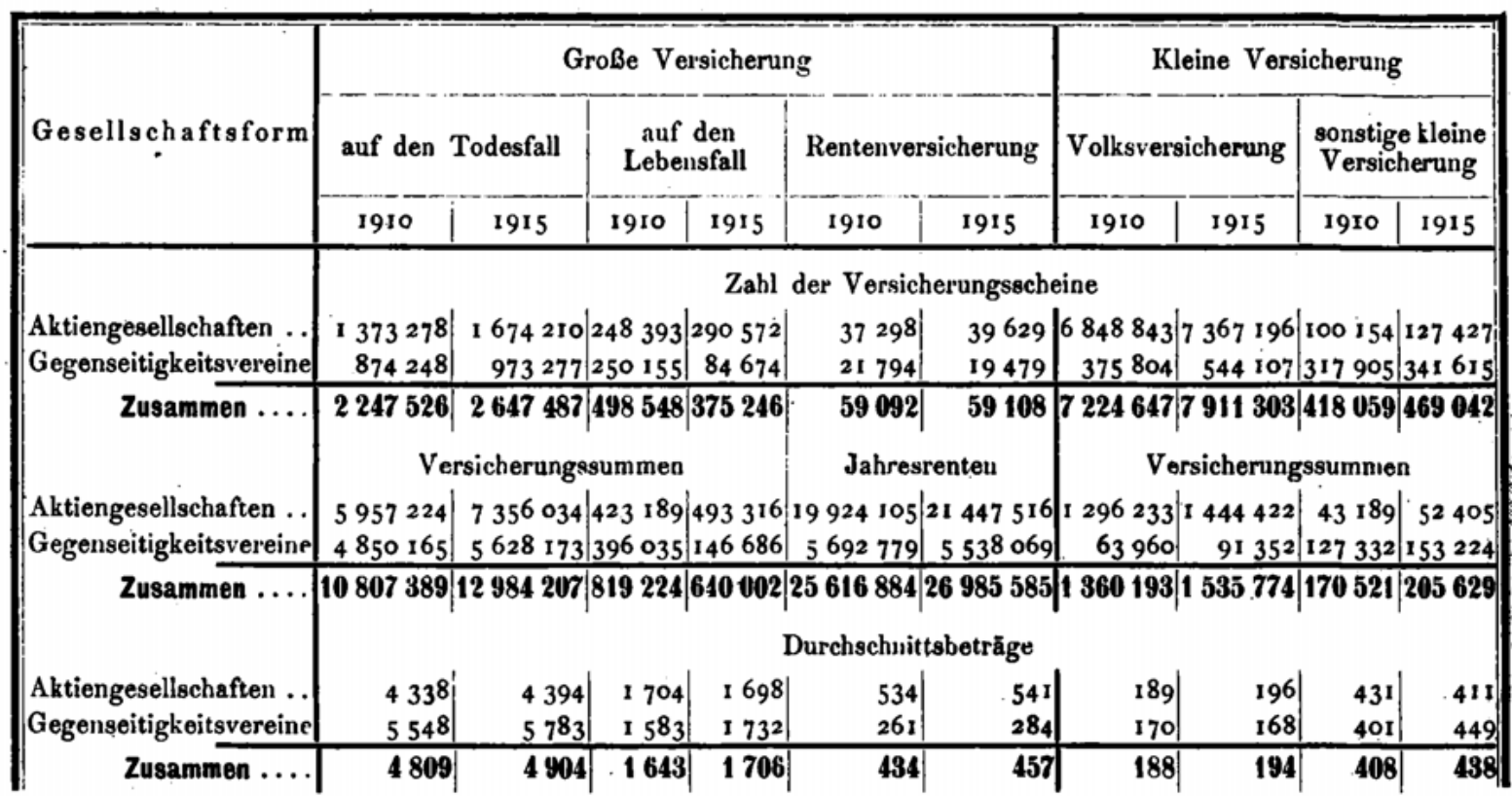

Die durchschnittlich auf einen Versicherungsschein entfallende Versicherungssumme ist in der großen Todesfallversicherung sowohl bei den Aktiengesellschaften wie auch bei den Gegenseitigkeitsvereinen gewachsen; sie ist bei den Gegenseitigkeitsvereinen fortdauernd höher als bei den Aktiengesellschaften. Der auf eine Versicherung auf den Lebensfall kommende durchschnittliche Betrag ist bei den Aktiengesellschaften etwas niedriger als bei den Gegenseitigkeitsvereinen; während der Durchschnittsbetrag bei den Aktiengesellschaften in den letzten Jahren etwas geschwankt hat, hat er bei den Gegenseitigkeitsvereinen ohne Unterbrechung zugenommen.

Beträchtlich größer waren die Durchschnittsbeträge bei der Gesamtheit der in den letzten Jahren ins Leben gerufenen öffentlichen Versicherungsanstalten. Die 11 Anstalten (10 Provinzialanstalten und der Verband der öffentlichen Lebensversicherungsanstalten), die für I9I5 einen Jahresbericht veröffentlicht haben, hatten Ende 19 I 5 im unmittelbaren Geschäfte zusammen 14643 Todesfallversicherungen über $106121721 \mathscr{M}, 474$ Versicherungen auf den Lebensfall über $2003267 \mathscr{M}, 196$ Versicherungen über $117137 \mathscr{N}$ Jahresrente und 55586 Volksversicherungen über $24423214 \mathscr{N}$ in Kraft. Die Durchschnittsbeträge stellen sich danach auf 7247 , auf 4226 , auf 598 und auf $439 \mathscr{M}$.

d) die Ver. sicherungen mit und obne Gewinnbeteiligung

In den Tafeln I 3 bis 7 und I 9 und 10 ist auch die Gewinnbeteiligung bei dem unmittelbaren Geschäfte behandelt. Daß Versicherungen mit Gewinnbeteiligung bei den Aktiengesellschaften verhältnismäßig weniger vorkommen als bei den Gegenseitigkeitsvereinen, liegt in der Natur der Gesellschaften. Genaueres hierüber ergibt für die letzten fünf Jahre die folgende Zusammenstellung.

Diese Zahlen zeigen durchweg einen sehr regelmäßigen Verlauf. In der Lebensfallversicherung und in der Rentenversicherung der Gegenseitigkeitsvereine sind die Versicherungen mit Gewinnbeteiligung während des betrachteten Zeitraums verhältnismäßig zurückgegangen, sowohl was die Zahl der Versicherungen als auch (wenigstens bei der Versicherung auf den Lebensfall) was die Höhe der Versicherungssummen betrifft. Bei 


\begin{tabular}{|c|c|c|c|c|c|c|c|c|c|c|}
\hline \multicolumn{11}{|c|}{ Am Gewinne waren im unmittelbaren Geschäfte beteiligt } \\
\hline \multirow{2}{*}{ Versicherungsart } & \multicolumn{5}{|c|}{ bei den Aktiengesellschaften } & \multicolumn{5}{|c|}{ bei den Gegenseitigkeitsvereinen } \\
\hline & 1911 & 1912 & 1913 & 1914 & 1915 & 1911 & 1912 & 1913 & 1914 & 1915 \\
\hline Große Versicherung & \multicolumn{10}{|c|}{. von je 1000 Versicherungsscheinen } \\
\hline Versicherung auf den Todesfall ........ & 901 & 905 & 909 & 909 & 908 & 995 & 994 & 993 & 993 & 992 \\
\hline$n \quad . \quad$ Lebensfall ....... & 336 & 330 & 324 & 317 & 545 & 906 & 900 & 897 & 892 & 775 \\
\hline Rentenversicherung $\ldots \ldots \ldots \ldots \ldots \ldots$ & $?$ & $?$ & $?$ & $?$ & $?$ & 742 & $73^{8}$ & 733 & 731 & 728 \\
\hline Kleine Versicherung & & & & & & & & & & \\
\hline Volksversicherung $\ldots \ldots \ldots \ldots \ldots \ldots$ & 732 & 741 & 747 & 747 & 747 & 940 & 934 & 925 & 917 & 889 \\
\hline Sonstige kleine Versicherung........... & 263 & 309 & 333 & $33^{2}$ & 320 & 931 & 933 & 935 & 936 & 935 \\
\hline Große Versicherung & \multicolumn{10}{|c|}{ von je $1000 \mathscr{A}$ Versicherungssumme (Jahresrente) } \\
\hline Versicherung auf den Todesfall ........ & 939 & 944 & 946 & 948 & 948 & 999 & 998 & 997 & 997 & 997 \\
\hline$\cdot \quad \cdot \quad$ Lebensfall ....... & 288 & 284 & 278 & 273 & 516 & 882 & 875 & 874 & $87 \mathrm{I}$ & 744 \\
\hline Rentenversicherung $\ldots \ldots \ldots \ldots \ldots \ldots$ & 17 & 17 & $21^{\circ}$ & 21 & 18 & 619 & 621 & 623 & 629 & 629 \\
\hline Kleine Versicherung & & & & & & & & & & \\
\hline Volksversicherung $\ldots \ldots \ldots \ldots \ldots \ldots \ldots$ & 810 & 819 & 829 & 829 & 823 & 972 & 972 & 969 & 966 & 952 \\
\hline Sonstige kleine Versicherung.......... & 301 & 322 & 344 & 353 & 349 & 928 & 932 & 934 & 935 & 933 \\
\hline
\end{tabular}

den Aktiengesellschaften ist infolge der erwähnten Fusionierung hinsichtlich der Lebensfallversicherung sowohl die Zahl der Versicherungen als auch die Höhe der Versicherungssummen nicht unerheblich gestiegen; bei der Rentenversicherung hat die Gewinnbeteiligung abgenommen, allerdings kommen solche Versicherungen mit Anteil am Gewinne bei den Aktiengesellschaften überhaupt nicht allzuoft vor. In der großen Versicherung auf den Todesfall ist der Anteil der gewinnberechtigten Versicherungen bei den Gegenseitigkeitsvereinen ziemlich ungeändert 'geblieben, bei den Aktiengesellschaften ist ein weiteres Steigen nicht mehr erfolgt. Bei der Volksversicherung der Aktiengesellschaften ist hinsichtlich der Versicherungssummen ein geringer Rückgang dieses Anteils zu verzeichnen.

Die Versicherungen mit Gewinnbeteiligung sind, wenn man von der kleinen Versicherung absieht, am häufigsten bei der Versicherung auf den Todesfall; hier nähern sich die Verhältniszahlen der Aktiengesellschaften denen der Gegenseitigkeitsvereine in bemerkenswerter Weise. Bei den Versicherungen auf den Lebensfall findet sich die Gewinnbeteiligung schon seltener, wenn auch der Unterschied bei den Gegenseitigkeitsvereinen kleiner ist als bei den Aktiengesellschaften. Beachtenswert ist vielleicht, daß bei beiden Arten von Unternehmungen in der Todesfallversicherung die Scheine der Zahl nach mit einem etwas geringeren Hundertsatz am Gewinn Anteil haben als die Versicherungssummen, während bei der Versicherung auf den Lebensfall das Entgegengesetzte der Fall ist. Die Versicherungen mit Anteil am Gewinne liegen also hinsichtlich der Höhe der Versicherung beim 'Todesfall über, beim Lebensfall unter dem Durchschnitte. Rentenversicherungen werden von den Aktiengesellschaften nur ausnahmsweise mit Gewinnbeteiligung der Versicherten abgeschlossen; von den Gegenseitigkeitsvereinen geschieht das naturgemäß viel häufiger.

Die Versicherung auf den Invaliditätsfall wird nur von wenigen Gesellschaften als selbständige Versicherung betrieben; zumeist tritt sie als Zusatzversicherung zu der gemischten Kapitalversicherung auf, indem für den Fall der Invalidität Beitragsfreiheit

e) Invaliditatsversicherung oder Beitragsfreiheit in Verbindung mit Gewährung einer Rente (meist 5 oder 10 v. H. des versicherten Kapitals) bis zum Ablaufe der Versicherung ausbedungen wird. Hin- 
sichtlich der hiermit verbundenen Unsicherheit in den Angaben über den Versicherungsbestand wird auf die Anmerkungen zu Tafel I 6 S. 20 Bezug genommen.

f) Kranken. veraicherung
Versicherungseinrichtungen ron Berufs-

vereinigungen

Die Krankenversicherung eignet sich weniger als jede andere Versicherungsart zum Betriebe von einer Zentrale aus; sie erfordert, wenn sie ohne übergroße Gefährdung des Versicherers durchgeführt werden soll, eine weitgehende Beobachtung der Ansprüche erhebenden Versicherten, die in einfacher Weise nicht zu ermöglichen ist. Tatsächlich befaßten sich im Jahre 1915 denn auch nur wenige Gesellschaften mit der Krankenversicherung; von diesen Gesellschaften wickeln 2 nur das von andern Gesellschaften übernommene Geschäft $a b$, ohne selbst neue Versicherungen abzuschließen, vgl. Tafel I 7.

Von den für begrenzte Kreise bestimmten Versicherungsunternehmungen sind, wie schon oben erwähnt, in die Tafeln dieser Statistik die Versicherungseinrichtungen einiger größerer Berufsvereinigungen aufgenommen, obwohl diese Einrichtungen sehr erhebliche Unterschiede zeigen und sich deshalb nicht ohne Zwang gemeinsam behandeln lassen.

Von den fraglichen 14 Unternehmungen betrieben 10 die Versicherung auf den Todesfall (meist als Sterbekassen), davon drei auch die Versicherung auf den Lebensfall und drei auch die Rentenversicherung (meist Pensionsversicherung); die übrigen vier befaßten sich lediglich mit der Pensionsversicherung.

Im ganzen waren bei diesen Versicherungseinrichtungen Ende 19 I 5 in Kraft 276102 Versicherungsscheine über $201358170 \mathscr{N}$ auf den Todesfall (durchschnittlich $729 \mathscr{N}$ für eine Versicherung), 606 Versicherungsscheine über $2337279 \mathscr{M}$ auf den Lebensfall (durchschnittlich $3857 \mathscr{N}$ für eine Versicherung). In der Rentenversicherung liefen zu Ende des Berichtsjahrs 13296 Versicherungsscheine über $3674566 \mathscr{N}$ Jahresrente (durchschnittlich $269 \mathscr{M}$ Jahresrente für eine Versicherung). Außerdem bestanden noch 85650 Pensionsversicherungen mit fast ausschließlich steigender Rente.

Bei der einfachen Verwaltung der meisten derartigen Versicherungsvereine ist es erklärlich, daß sie bis auf die Deutsche Beamten-Lebensversicherung A.-G. in Berlin in der Rentenversicherung keine Versicherungen in Rückdeckung übernommen haben. Auch die in Rückdeckung gegebenen Beträge sind nicht eben beträchtlich (vgl. Anm. zu Taf̣. I11 S. 26).

Von den ausländischen Versicherungsunternehmungen, die im Jahre 1915 im Deutschen Reiche Versicherungsgeschäfte betrieben haben, soll hier nur der Bestand des deutschen Geschäfts von I7 Gesellschaften erörtert werden, wie er in den Tafeln I 12 bis I 18 erscheint. Dabei wird von einer Trennung zwischen Aktiengesellschaften und Gegenseitigkeitsvereinen abgesehen.

Wie vorhin bei den deutschen allgemeinen Unternehmungen, betrachten wir zunächst den gesamten übernommenen Bestand und den davon für eigene Rechnung behaltenen Teil.

Es waren

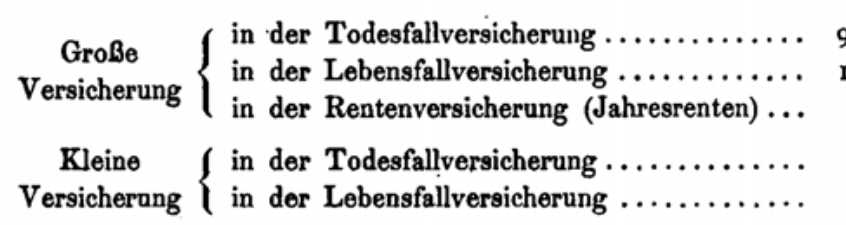

überhaupt

übernommen

$932398820 \mu$

114302014 .

1488017 .

II 795678 *

50303 . von $1000 \varkappa$ úberhaupt übernommener Versicherung für eigene Rechnung behalten $948 \varkappa$ 990 . $944 \cdot$

$982 \cdot$ 1000 . 
Danach haben die ausländischen Gesellschaften nur in der großen Todesfallversicherung und in der Rentenversicherung einen nennenswerten Betrag in Rückdeckung gegeben. Aus der kleinen Versicherung haben sie nur in der Todesfallversicherung einen geringen Betrag rückversichert (Quotenrückversicherung).

Uber das deutsche unmittelbare Geschäft der ausländischen Unternehmungen gibt sodann folgende Zusammenstellung Auskunft.

Deutsches unmittelbares Geschäft der ausländischen Unternehmungen

Versicherungssummen in $1000 \mathscr{M}$, Jahresrenten und Durchschnittsbeträge in $\mathscr{M}$

\begin{tabular}{|l|r|r|r|r|}
\hline & \multicolumn{2}{|c|}{ Große Versicherung } \\
& $\begin{array}{r}\text { auf den } \\
\text { Todesfall }\end{array}$ & $\begin{array}{r}\text { auf den } \\
\text { Lebensfall }\end{array}$ & $\begin{array}{r}\text { Renten- } \\
\text { versicherung }\end{array}$ & $\begin{array}{r}\text { Volks- } \\
\text { versicherung }\end{array}$ \\
\hline & 1915 & 1915 & 1915 & 1915 \\
\hline Zahl der Versicherungsseheine...... & 148449 & 80794 & 2159 & 47864 \\
Versicherungssummen (Jahresrenten) & 909189 & 114235 & 1471558 & 11846 \\
Durchschnittsbeträge ............... & 6125 & 1414 & 682 & 274 \\
\hline
\end{tabular}

Die Durchschnittsbeträge sind bei den ausländischen Gesellschaften in der großen Todesfallversicherung merklich höher als bei den deutschen Anstalten; das rührt jedoch daher, daß einige wenige Gesellschaften besonders hohe Beträge übernommen haben; es handelt sich nicht um eine Eigentümlichkeit aller ausländischen Unternehmungen.

Uber das Verhältnis der mit Gewinnbeteiligung der Versicherten abgeschlossenen Verträge zu den überhaupt abgeschlossenen Verträgen und über die Entwickelung dieses Verhältnisses in den letzten fünf Jahren ergeben sich bei Beschränkung auf das unmittelbare Geschäft folgende Zahlen, wobei indessen zu beachten ist, daß seit I 9 I 4 zwei englische und zwei französische Gesellschaften fehlen.

Im deutschen unmittelbaren Geschäfte der ausländischen Unternehmungen waren am Gewinne beteiligt:

\begin{tabular}{|c|c|c|c|c|c|}
\hline Versicherungsart & I9II & 1912 & 1913 & 1914 & 1915 \\
\hline & \multicolumn{5}{|c|}{ von je 1 ooo Versicherungsscheinen } \\
\hline Große Todesfallversicherung $\ldots \ldots \ldots \ldots \ldots$ & 855 & 862 & 868 & 870 & 869 \\
\hline - Lebensfallversicherung $\ldots \ldots \ldots \ldots \ldots$ & 827 & 835 & 842 & 846 & 989 \\
\hline Rentenversicherung $\ldots \ldots \ldots \ldots \ldots \ldots \ldots$ & 47 & 43 & 41 & 39 & 37 \\
\hline \multirow[t]{2}{*}{ Volksversicherung $\ldots \ldots \ldots \ldots \ldots \ldots \ldots$} & 124 & III & 117 & 116 & 108 \\
\hline & \multicolumn{5}{|c|}{ von je I $000 \mathscr{M}$ Versicherungssumme } \\
\hline Große Todesfallversicherung $\ldots \ldots \ldots \ldots \ldots$ & 907 & 911 & 914 & 916 & 915 \\
\hline ; Lebensfallversicherung $\ldots \ldots \ldots \ldots \ldots$ & 778 & 782 & 789 & 762 & 764 \\
\hline Rentenversicherung $\ldots \ldots \ldots \ldots \ldots \ldots \ldots$ & 42 & 43 & 47 & 48 & 45 \\
\hline Volksversicherung $\ldots \ldots \ldots \ldots \ldots \ldots \ldots$ & 401 & 368 & 362 & 354 & 339 \\
\hline
\end{tabular}

Wie bei den deutschen Gesellschaften, ist auch bei den ausländischen Unternehmungen die Gewinnbeteiligung derVersicherten bei der Todesfallversicherung am häufigsten. Bezüglich der Versicherungssumme ist die Gewinnbeteiligung der Versicherten bei den ausländischen Anstalten geringer als bei den deutschen Aktiengesellschaften. 
In der Volksversicherung schließen die ausländischen Gesellschaften bei uns verhältnismäßig sehr viel weniger Verträge mit Gewinnbeteiligung ab als die deutschen Unternehmungen.

\section{Die Bewegung im Versicherungsbestand im Jahre 1915}

Doutache allgemeine Unternehmungen

Die Bewegung im Versicherungsbestande der deutschen Unternehmungen, die allen Kreisen der Bevölkerung offenstehen, ist in den Tafeln I 19 bis I 23 ausführlich' behandelt. Dabei hat aber, wie schon oben bemerkt ist, die 'Trennung zwischen großer und kleiner Versicherung nicht so vollständig durchgeführt werden können wie bei der Beschreibung des Bestandes am Ende des Berichtsjahrs; die Bewegung der Sterbegeldversicherung war bei einigen Unternehmungen nicht aus der Bewegung der großen Versicherung auszuscheiden.

Die folgende Zusammenstellung gibt einen Uberblick über die Bewegung der wichtigsten Versicherungsarten in dem gesamten Geschäfte der großen deutschen Unternehmungen; dabei ist bei der Volksversicherung. und bei der sonstigen kleinen Versicherung die Todesfallversicherung mit der Lebensfallversicherung zusammengefaßt. In Betracht gezogen sind nur die selbst abgeschlossenen Versicherungen.

Der früher gewohnte Fortschritt ist weiter durch den Krieg unterbrochen worden; auch 1915 hat der Abgang den Zugang überall noch übertroffen. Ier Abgang ist, wie

Bewegung im Versicherungsbestande der deutschen Unternehmungen

\begin{tabular}{|c|c|c|c|c|c|c|c|c|c|c|}
\hline & \multicolumn{2}{|c|}{$\begin{array}{c}\text { Versicherung } \\
\text { auf } \\
\text { den Todesfall }\end{array}$} & \multicolumn{2}{|c|}{\begin{tabular}{|c|} 
Versicherung \\
auf \\
- den Lebensfall \\
$\begin{array}{l}\text { Versiche- } \\
\text { rungs- } \\
\text { selieine }\end{array} \mid 1000 \stackrel{N}{ }$ \\
\end{tabular}} & \multicolumn{2}{|c|}{$\begin{array}{c}\text { Renten- } \\
\text { versicherung } \\
\begin{array}{c}\text { Versiche- } \\
\text { rungs- } \\
\text { seheine }\end{array} \mid 1000 \AA\end{array}$} & \multicolumn{2}{|c|}{$\begin{array}{c}\text { Volks- } \\
\text { versicherung }\end{array}$} & \multicolumn{2}{|c|}{$\begin{array}{l}\text { Sonstige kleine } \\
\text { Versicherung }\end{array}$} \\
\hline \multicolumn{11}{|c|}{ Aktiengesellschaften } \\
\hline & \multicolumn{2}{|c|}{$\begin{array}{l}25 \text { Gesell- } \\
\text { schaften }\end{array}$} & \multicolumn{2}{|c|}{$\begin{array}{l}24 \text { Gesell- } \\
\text { schaften }\end{array}$} & \multicolumn{2}{|c|}{$\begin{array}{l}22 \text { Gesell- } \\
\text { schaften }\end{array}$} & \multicolumn{2}{|c|}{$\begin{array}{c}12 \text { Gesell- } \\
\text { schaften }\end{array}$} & \multicolumn{2}{|c|}{$\begin{array}{l}11 \text { Gesell- } \\
\text { schaften }\end{array}$} \\
\hline Bestand Ende 1914 & I 771579 & $7607 \times 27$ & 322171 & 538466 & 40288 & 21936 & 7677684 & 1530894 & 95318 & 36535 \\
\hline Zugang .......... & 44877 & 238698 & 4475 & so 756 & I 639 & 760 & 400190 & 99452 & 4711 & 963 \\
\hline Abgang ......... & 106702 & 472489 & 36074 & 55906 & 2298 & 1249 & 710678 & 185924 & 8146 & 3396 \\
\hline Reiner Zugang... & -61825 & -233791 & -31599 & $-45 \quad 150$ & -659 & -489 & -310488 & -86472 & -3435 & -1433 \\
\hline \multirow[t]{3}{*}{ Bestand Ende 1915 } & $|1709754|$ & 7373336 & $|290572|$ & $\mid 493316$ & $|39629|$ & $|21447|$ & $|7367196|$ & $\mid$ I $444422 \mid$ & $|91883|$ & 35102 \\
\hline & \multicolumn{8}{|c|}{ Gegenseitigkeltsvereine } & & \\
\hline & \multicolumn{2}{|c|}{15 Vereine } & \multicolumn{2}{|c|}{12 Vereine } & \multicolumn{2}{|c|}{12 Vereine } & \multicolumn{2}{|c|}{4 Vereine } & \multicolumn{2}{|c|}{7 Vereine } \\
\hline Bestand Ende 1914 & 994313 & 5673519 & 90617 & 156814 & 20157 & 5706 & 555298 & 94295 & 346795 & 157119 \\
\hline Zugang...$\ldots \ldots$ & 24579 & 196578 & 1971 & $4 \mathrm{I} 4 \mathrm{I}$ & $33^{8}$ & 105 & 53285 & 8022 & 4429 & 3282 \\
\hline Abgang...$\ldots \ldots$ & 42783 & 240272 & 7914 & 14270 & 1016 & 273 & 64476 & 10965 & $1244 \mathrm{I}$ & 8829 \\
\hline Reiner Zugang ... & - 18204 & -43694 & $-5943 \mid$ & $\begin{array}{lll}-10 & 129\end{array}$ & -678 & -168 & $\begin{array}{lll}-11 & 191\end{array}$ & -2943 & -8012 & -5547 \\
\hline Bestand Ende 1915 & $|976109|$ & 5629825 & 84674 & 146685 & $19479 \mid$ & $\left|553^{8}\right|$ & 544107 & $91352 \mid$ & $33^{8} 783$ | & 1511572 \\
\hline \multicolumn{11}{|c|}{ Sămtliche Unternehmungen } \\
\hline & \multicolumn{2}{|c|}{$\begin{array}{l}40 \text { Unter- } \\
\text { nehmụngen }\end{array}$} & \multicolumn{2}{|c|}{$\begin{array}{c}36 \text { Unter- } \\
\text { nehmungen }\end{array}$} & \multicolumn{2}{|c|}{$\begin{array}{c}34 \text { Unter- } \\
\text { nehmungen }\end{array}$} & \multicolumn{2}{|c|}{$\begin{array}{l}16 \text { Unter- } \\
\text { nehmungen }\end{array}$} & \multicolumn{2}{|c|}{$\begin{array}{l}18 \text { Unter- } \\
\text { nehmungen }\end{array}$} \\
\hline Bestand Ende 1914 & 2765892 & 13280646 & 412788 & 695280 & $\begin{array}{ll}60 & 445\end{array}$ & 27642 & 8232982 & 1625189 & 442 I 13 & 193654 \\
\hline Zugang .......... & $6945^{6}$ & 435276 & 6446 & เ4 897 & I 977 & 865 & 453475 & 107474 & 9140 & 5245 \\
\hline bgang ......... & 149485 & 712761 & 43988 & 70176 & 3314 & 1522 & 775154 & 196889 & $20587 \mid$ & 12225 \\
\hline $\begin{array}{l}\text { Reiner Zugang .... } \\
\text { Bestand Ende } 19{ }_{5} 5\end{array}$ & $\left|\begin{array}{rr}-80029 \\
2685863\end{array}\right|$ & $\begin{array}{r}-277485 \\
13003161\end{array}$ & \begin{tabular}{|}
-37542 \\
375246
\end{tabular} & $\begin{array}{rr}-55 & 279 \\
640001\end{array} \mid$ & $\mid \begin{array}{rr}-1 & 337 \\
59 & 108\end{array}$ & $\left|\begin{array}{l}-657 \\
26985\end{array}\right|$ & $\left|\begin{array}{l}-321679 \\
7911303\end{array}\right|$ & $\left|\begin{array}{r}-89415 \\
1535774\end{array}\right|$ & 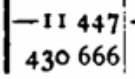 & $\begin{array}{l}-16980 \\
1816674\end{array}$ \\
\hline
\end{tabular}


im Jahre 1914, durch die Kriegstodesfälle stark in die Höhe gegangen, und der Zugang ist viel kleiner gewesen als sonst. Der Krieg hat durch Einberufung zum Heeresdienste große Lücken in das Netz der Vertreter und Vermittler der Gesellschaften gerissen und die Kreise, in denen vornehmlich für Lebensversicherungen geworben wurde, gelichtet. Die Ausscheidenden kamen zunächst für die Versicherung nicht mehr in Betracht.

Wegen der großen Bedeutung, die den verschiedenen Ursachen des Abgangs von Versicherungen zukommt, sind in unsern Tafeln für die Hauptversicherungsarten nicht nur die absoluten Zahlen für die Häufigkeit der einzelnen Ursachen angegeben, sondern es ist auch berechnet, wieviel bei den selbst abgeschlossenen Versicherungen von 1000 Scheinen oder von $1000 \mathscr{M}$ Versicherungssumme des durchschnittlichen Bestandes durch Tod, wieviel durch Ablauf usw. in Wegfall gekommen sind.

Mehr als in andern Zeiten verdienen während des Kriegs diese Zahlen unsere Aufmerksamkeit. Zu Friedenszeiten schienen sie von Jahr zu Jahr fast unveränderlich; jetzt bewegen sie sich, vornehmlich natürlich in der Versicherung auf den Todesfall. Wir geben daher folgende kurze Zusammenstellung, die für sich selber spricht.

\begin{tabular}{|c|c|c|c|c|c|c|}
\hline & $\begin{array}{l}\text { Abgang du } \\
\text { Ver- } \\
\text { sicherungs- } \\
\text { scheine }\end{array}$ & $\begin{array}{l}\text { arch Tod } \\
\text { Summen }\end{array}$ & $\begin{array}{l}\text { Storno mit } \\
\text { Ver- } \\
\text { sicherungs- } \\
\text { scheine }\end{array}$ & $\begin{array}{l}\text { Vergütung } \\
\text { Summen }\end{array}$ & $\begin{array}{l}\text { Storno ohne } \\
\text { Ver- } \\
\text { sicherungs- } \\
\text { scheine }\end{array}$ & $\begin{array}{l}\text { Vergütung } \\
\text { Summen }\end{array}$ \\
\hline \multicolumn{7}{|l|}{ GroßeVersicherung auf denTodesfall } \\
\hline Aktiengesellschaften ........ 1914 & 15 & 14 & 15 & 18 & 23 & 23 \\
\hline $\begin{array}{lll}1915 & \end{array}$ & 21 & 18 & 16 & 18 & 15 & 17 \\
\hline Gegenseitigkeitsvereine ..... 1914 & 18 & 18 & 8 & 9 & 9 & 7 \\
\hline igrs & 22 & 20 & 6 & 8 & 5 & 5 \\
\hline \multicolumn{7}{|l|}{ Volksversicherung } \\
\hline Aktiengesellschaften ........ 1914 & I I & 10 & 7 & 27 & 71 & 89 \\
\hline 1915 & 14 & 14 & 5 & 35 & 44 & 50 \\
\hline Gegenseitigkeitsveroine ..... I9I4 & 10 & 10 & 35 & 35 & 120 & 142 \\
\hline 1915 & 14 & 14 & 23 & 25 & 61 & 66 \\
\hline
\end{tabular}

Wenn man bei den Versicherungseinrichtungen der größeren Berufsvereinigungen (Pensionskassen und Sterbekassen) diejenigen Kassen außer Betracht läßt, bèi denen die Jahresrenten als veränderlich nicht angegeben werden können, erhält man folgendes Bild von der Bestandsänderung im Berichtsjahre:

\begin{tabular}{|c|c|c|c|c|}
\hline & \multicolumn{2}{|c|}{$\begin{array}{c}\text { Kapitalversicherung } \\
10 \text { Vereine }\end{array}$} & \multicolumn{2}{|c|}{$\begin{array}{c}\text { Rentenversicherung } \\
3 \text { Vereine }\end{array}$} \\
\hline & $\begin{array}{l}\text { Ver- } \\
\text { sicherungs- } \\
\text { scheine }\end{array}$ & 1000 A A & $\begin{array}{l}\text { Ver- } \\
\text { sicherungs- } \\
\text { scheine }\end{array}$ & $\begin{array}{c}1000 \mathscr{N} \\
\text { Rente }\end{array}$ \\
\hline Bestand Ende $1914 \ldots \ldots \ldots \ldots \ldots \ldots$ & 280856 & 206963 & 13591 & 3723 \\
\hline Zugang $\ldots \ldots \ldots \ldots \ldots \ldots \ldots \ldots \ldots \ldots$ & 4033 & 3676 & 95 & 45 \\
\hline Abgang $\ldots \ldots \ldots \ldots \ldots \ldots \ldots \ldots \ldots \ldots$ & $8 \mathrm{I} 8 \mathrm{I}$ & 6944 & 390 & 93 \\
\hline Reiner Zugang $\ldots \ldots \ldots \ldots \ldots \ldots \ldots$ & -4148 & -3268 & -295 & -48 \\
\hline Bestand Ende $1915 \ldots \ldots \ldots \ldots \ldots \ldots$ & 276708 & 203695 & 13296 & 3675 \\
\hline
\end{tabular}

Die Bewegung im deutschen Versicherungsbestande der ausländischen Lebensversicherungsunternehmungen ist in den Tafeln I 25 bis I 28 ausführlich dargestellt. Aus diesen Tafeln ist folgende Ubersicht hergeleitet: 
Gruppe I

Bewegung im deutschen Versicherungsbestand der ausländischen Gesellschaften

\begin{tabular}{|c|c|c|c|c|c|c|c|c|}
\hline & \multicolumn{2}{|c|}{$\begin{array}{l}\text { Versicherung auf } \\
\text { den Todesfall } \\
17 \text { Gesellschaften }\end{array}$} & \multicolumn{2}{|c|}{$\begin{array}{l}\text { Versicherung auf } \\
\text { den Lebensfall } \\
16 \text { Gesellschaften }\end{array}$} & \multicolumn{2}{|c|}{$\begin{array}{c}\text { Renten- } \\
\text { versicherung } \\
16 \text { Gesellschaften }\end{array}$} & \multicolumn{2}{|c|}{$\begin{array}{c}\text { Volks- } \\
\text { versicherung } \\
3 \text { Gesellschaften }\end{array}$} \\
\hline & $\begin{array}{c}\begin{array}{c}\text { Ver- } \\
\text { sicherungs- } \\
\text { scheine }\end{array} \\
\end{array}$ & $1000 \mathscr{A}$ & $\begin{array}{c}\text { Ver- } \\
\text { sicherungs- } \\
\text { scheine }\end{array}$ & $1000 \varkappa$ & $\begin{array}{c}\text { Ver- } \\
\text { sicherungs- } \\
\text { scheine }\end{array}$ & $1000 \mathscr{M}$ & $\begin{array}{c}\text { Ver- } \\
\text { sicherungs- } \\
\text { scheine }\end{array}$ & $1000 \mu$ \\
\hline Bestand Ende $1914 \ldots \ldots \ldots$ & I 53354 & 946398 & 82526 & 119502 & 2206 & I 508 & 47179 & 12000 \\
\hline Zugang................. & 5394 & 19256 & 4319 & 4826 & 48 & 42 & 6776 & 1254 \\
\hline Abgang $\ldots \ldots \ldots \ldots \ldots \ldots$ & 10109 & 56436 & $605 \mathrm{r}$ & 10093 & 95 & 78 & 6281 & $1+37$ \\
\hline Reiner Zugang $\ldots \ldots \ldots \ldots$ & -4715 & -37 I8o & $-173^{2}$ & -5267 & $-\quad 47$ & -36 & 495 & -183 \\
\hline Bestand Ende $1915 \ldots \ldots \ldots$ & 148639 & 909218 & 80794 & 114235 & 2159 & I 472 & 47674 & 11817 \\
\hline
\end{tabular}

Uber die Häufigkeit der verschiedenen Arten des Abganges seien hier die folgenden Zahlen aufgeführt, die den oben für die deutschen Unternehmungen angegebenen Zahlen entsprechen.

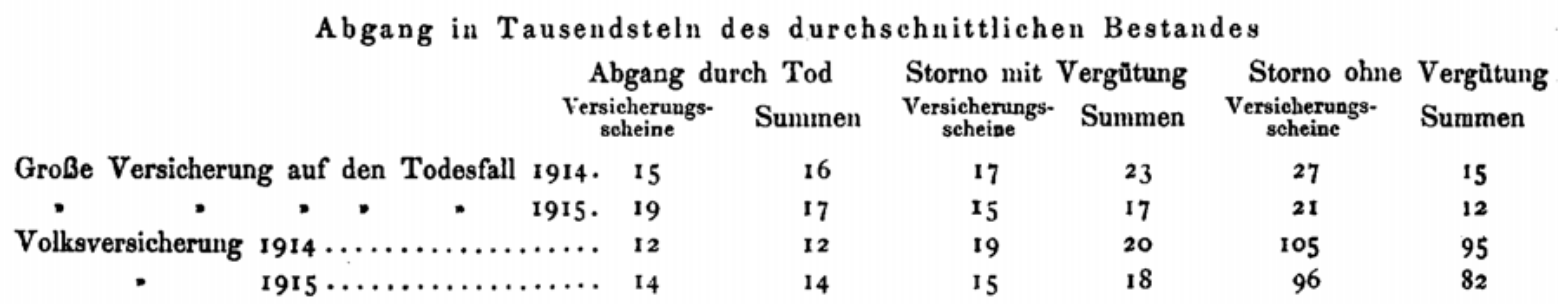

\section{Die Betriebsrechnung für 1915}

Allgemeines Die Gewinn- und Verlustrechnung, wie sie in den Tafeln I 29, 31, 33 und 35 gegeben ist, unterscheidet sich von den Aufstellungen, welche die Unternehmungen nach dem Formular L 1 der Rechnungsvorschriften dem Aufsichtsamt einzureichen haben, dadurch, daß die veränderlichen Rücklagen (Prämienreserve, Prämienüberträge, Rücklagen für schwebende Versicherungsfälle, Gewinnrücklagen und sonstige Rücklagen) nicht mit ihren Beständen zu Anfang des Jahres in Einnahme und mit ihren Werten am Ende des Jahres in Ausgabe gestellt sind, sondern daß nur die durch den Jahresbetrieb hervorgerufenen Änderungen nachgewiesen sind; denn nur diese Änderungen beeinflussen das Ergebnis der Jahresrechnung; außerdem wird die Rechnung durchsichtiger. Der Stand der Rücklagen am Ende des Jahres findet sich in der Bilanz. Sodann ist der Vortrag aus dem Überschusse des Vorjahrs hier fortgelassen worden, um das Ergebnis des Geschäftsbetriebs lediglich aus dem Berichtsjahre zu erhalten.

Die Betriebsrechnung bezieht sich auf das ganze (deutsche und ausländische) Lebensversicherungsgeschäft der Unternehmungen. Für die Volksversicherung ist bei den deutschen Gesellschaften eine besondere Betriebsrechnung (Tafel I 31) aufgestellt; die sonstige kleine Versicherung und bei den ausländischen Gesellschaften auch die Volksversicherung ist mit dem Geschäft in der großen Versicherung zusammen verrechnet (Tafel I 29 und 35). Auch sind beim Atlas, bei der Wilhelma und bei der Bayer. V. B. die geringen Einnahmen und Ausgaben der Kautionsversicherung in der allgemeinen Betriebsrechnung für das gesamte Lebensversicherungsgeschäft enthalten (vgl. die Anmerkungen 1 und 2 zu Tafel I 30 S. 92 und 93). 
Die in den Utbersichten über die Gewinn- und Verlustrechnung nur im Ganzen eingestellten Beiträge und Vergütungen der Rückversicherer einerseits und die Zahlungen für selbst abgeschlossene und für in Rückdeckung übernommene Versicherungen sowie die Rückversicherungsbeiträge anderseits sind für die deutschen Unternehmungen in den Tafeln I 30, 32 und 34 erläutert. Für die ausländischen Gesellschaften sind die entsprechenden Angaben nur für den deutschen Geschäftsbetrieb zusammengestellt (Tafel I 36).

In der Lebensversicherung ohne Volksversicherung haben bei den deutschen Unternehmungen betragen:

\begin{tabular}{|c|c|c|c|}
\hline & $\begin{array}{l}\text { Gesamtheit der } \\
\text { Unternehmungen }\end{array}$ & $\begin{array}{l}26 \text { Aktien- } \\
\text { gesellschaften }\end{array}$ & $\begin{array}{c}16 \text { Gegenseitigkeits } \\
\text { vereine }\end{array}$ \\
\hline die Summe der Einnahmen ......... & $896596534 \mathscr{M l}$ & $522923423 \mathscr{N}$ & $373673111 \mu$ \\
\hline die Summe der Ausgaben..$\ldots \ldots \ldots$ & 749439596 & 441253078 & 308186518 \\
\hline der Jahresüberschuß ... & $147156938 \mathscr{H}$ & $81670345 \mathscr{A l}$ & $65486593 \mathscr{M}$ \\
\hline
\end{tabular}

Das Geschäftsjahr I9I5 hat 25 Aktiengesellschaften in der großen Lebensversicherung einen Uberschuß von zusammen $81743773 \mathscr{N}$ gebracht; eine Gesellschaft, die übrigens nur Krankenversicherung (auch mit Einschluß von Sterbegeld) betrieben hat, hat einen Verlust von $73428 \mathscr{M}$ : ausgewiesen. Bei den 16 Gegenseitigkeitsvereinen ist ein Gewinn von zusammen $65486593 \mathscr{M}$ erzielt worden. Von dem durch die Bilanz ausgewiesenen Gewinn und dessen Verteilung wird unten gesprochen werden.

Für die Volksversicherung ergeben sich folgende Zahlen:

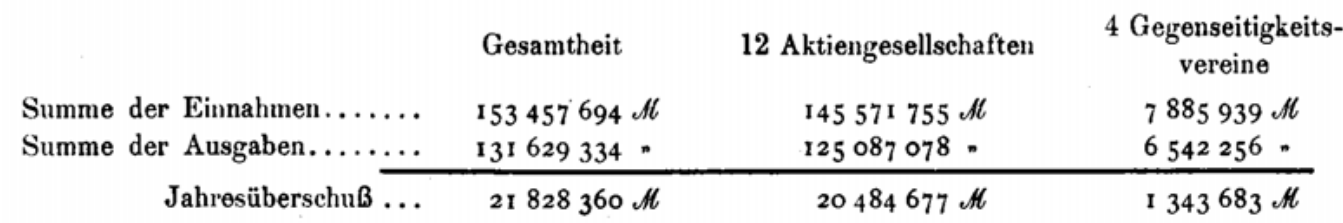

Die 12 Aktiengesellschaften, deren Betriebsrechnung für das Volksversicherungsgeschäft in Tafel I 31 aufgestellt ist, haben im Jahre 19I5 mit einem Gewinne von $20484677 \mathscr{M}$ gearbeitet. Die vier Gegenseitigkeitsvereine haben einen Uberschuß von zusammen $1343683 \mathscr{N}$ erzielt.

Für eingetretene Versicherungsfälle des Geschäftsjahrs aus selbst abgeschlossenen Versicherungen wurden im Berichtsjahr in der großen Versicherung von der Gesamtheit der Unternehmungen 89,2 v. H. der fällig gewordenen Summen ausbezahlt und 10,8 v. H. zurückgestellt; im besonderen von den Aktiengesellschaften 89, 3 und 10,7 v. H., von den Gegenseitigkeitsvereinen 89,1 und 10,9 v. H. In der Volksversicherung wurden von der Gesamtheit der Unternehmungen 92,54 v. H. aller fällig gewordenen Versicherungssummen gezahlt und 7,46 v. H. zurückgestellt; im besonderen bei den Aktiengesellschaften 92,73 und 7,27 v. H., bei den Gegenseitigkeitsvereinen 87,56 und $12,44 \mathrm{v}$. H. Bei Vergleichen mit den früheren Jahren ist zu beachten, daß die Zahlungen aus Versicherungsfällen bei der Victoria seit 1909 die mit den Versicherungssummen ausgezahlten Gewinnanteile nicht mehr enthalten; diese erheblichen Beträge (im Berichtsjahre nach Anmerkung ${ }^{4}$ ) S. 99: 13255900 Al ) sind vielmehr bei den an die Versicherten ausgezahlten Gewinnanteilen verrechnet. 
Versicherungseinrichtun. gen von Berufsver einigungen

Uber die Gewinn- und Verlustrechnung der in diese Statistik aufgenommenen Versicherungseinrichtungen der größeren Berufsvereinigungen geben die Tafeln I 33 und I 34 Aufschluß.

Daraus sind folgende Zahlen zu entnehmen:

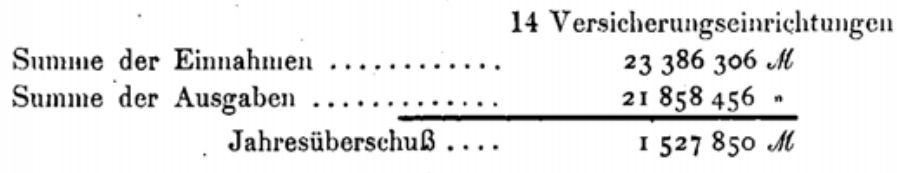

Finen Fehlbetrag haben im Berichtsjahre zwei dieser Anstalten mit $195779 \mathscr{N}$ ausgewiesen; die übrigen 12 Anstalten erzielten einen Überschuß von zusammen $1723629 \mathscr{M}$.

Zur Beurteilung des Geschäftsbetriebs derjenigen ausländischen Lebensversicherungsgesellschaften, aber welche diese Statistik berichtet, ist in Tafel I35 die Gewinnund Verlustrechnung für das ganze inländische und ausländische Geschäft möglichst nach denselben Grundsätzen dargestellt, die bei den deutschen Gesellschaften angewandt worden sind. Daneben sind aber in Tafel I 36 die Einnahmen und Ausgaben der ausländischen Gesellschaften allein aus dem deutschen Geschäfte zusammengestellt. Es haben betragen die Einnahmen an Beiträgen im deutschen Geschäft insgesamt $45424422 \mathscr{M}$. Davon kommen $44588453 \mathscr{M}$ oder 98,2 v. H. auf selbst abgeschlossene Versicherungen und $835969 \mathscr{M}$ oder 1,8 v. H. auf die in Rückdeckung übernommenen Versicherungen. Für Versicherungsleistungen einschließlich der Zahlungen für vorzeitig aufgelöste Versicherungen waren für das deutsche Geschäft bereitzustellen insgesamt $39349975 \mathscr{N}$, davon $38604924 \mathscr{M}$ oder 98,1 v. H. für selbst abgeschlossene Versicherungen und $745051 \mathscr{M}$ oder 1,9 v. H. für in Rückdeckung übernommene Versicherungen.

\section{Die Bilanz für den Schluß des Jahres 1915}

Eine Bilanz ist in dieser Statistik grundsätzlich für das Gesamtgeschäft einer jeden Gesellschaft aufgestellt und bei derjenigen Gruppe nachgewiesen, zu der das Unternehmen seinem Hauptbetriebe nach gehört.

Dementsprechend sind in Tafel I 37 die Bilanzen für 28 Aktiengesellschaften und 17 Gegenseitigkeitsvereine aufgeführt; die Gesamtbilanz schließt für Ende I 9 I 5 bei den Aktiengesellschaften mit $4206145307 \mathscr{N}$ und bei den Gegenseitigkeitsvereinen mit $2419230405 \mathscr{N} \mathrm{ab}$; als Hauptposten sind folgende Beträge zu verzeichnen:

\begin{tabular}{|c|c|c|}
\hline \multicolumn{2}{|c|}{ Vermögenswerte $\quad 28$ Aktiengesellschaften } & \multirow{2}{*}{17 Gegenseitigkeitsvereine } \\
\hline Verpflichtungen der Aktionäre oder Garanten.......... & $120086221 \mathscr{M}$ & \\
\hline 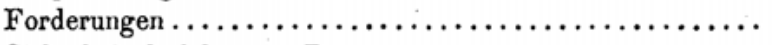 & $21948255^{8}$ & 84543049 \\
\hline Sicherheitsdarlehen an Beamte...$\ldots \ldots \ldots \ldots \ldots \ldots$ & $201006 n$ & $942019 n$ \\
\hline 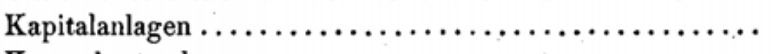 & $3835405478 n$ & $2309917359 "$ \\
\hline Kassenbestand $\ldots \ldots \ldots \ldots \ldots \ldots \ldots \ldots \ldots \ldots \ldots \ldots \ldots \ldots$ & $3221.474 "$ & $3186170 "$ \\
\hline Verbindlichkeiten & & \\
\hline Aktien- oder Garantiekapital..................... & $162672857 \mathscr{M}$ & $423371 \mathscr{H}$ \\
\hline Gesetzliche Rücklage $\ldots \ldots \ldots \ldots \ldots \ldots \ldots \ldots \ldots \ldots$ & $16294397 n$ & II 572298 n \\
\hline 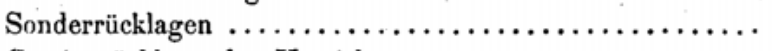 & $137.988171=$ & $48972943 n$ \\
\hline Gewinnrücklage der Versicherten.................. & $360542629 \cdots$ & 215829779 \\
\hline Guthaben von Versicherungsunternehnungen $\ldots \ldots \ldots \ldots$ & 17667024 & $773694 \cdots$ \\
\hline 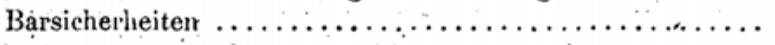 & 2485336 & $907602 n$ \\
\hline Prämienreserven und Prämienüberträge $\ldots \ldots \ldots \ldots \ldots \ldots$ & $3233337777 "$ & I 953 131 928 " \\
\hline Rücklage für schwebende Versicherungsf älle $\ldots \ldots \ldots \ldots$. & 54004232 & $24990977 n$ \\
\hline
\end{tabular}


Die hier aufgeführten Kapitalanlagen verteilen sich auf folgende Wertarten ('Tafel I38):

\begin{tabular}{|c|c|c|c|c|c|c|c|}
\hline \multirow[b]{2}{*}{ Grundbesit\% $\ldots \ldots \ldots \ldots \ldots \ldots \ldots \ldots$} & \multicolumn{3}{|c|}{23 Aktiengesellschaften } & \multicolumn{4}{|c|}{17 Gegenseitigkeitsvereine } \\
\hline & 92935387 & $\mathscr{A l}$ oder & $2,4 \%$ & 17815548 & $\mathscr{M}$ oder & 0,8 & $\%$ \\
\hline Hypotheken.$\ldots \ldots \ldots \ldots \ldots \ldots \ldots \ldots$ & 3014864033 & $" \quad$, & 78,6 & 1736517347 & $" \quad \cdots$ & 75,2 & $n$ \\
\hline Darlehen an öffentliche Körperschaften & 107000440 & n & 2,8 & 188 o I I 600 & " & $8, \mathrm{I}$ & $\cdot$ \\
\hline Darlehen auf Versicherungen ......... & 309883539 & $"$ & $8, \mathrm{r}$ & 208215628 & $n$ & 9,0 & $"$ \\
\hline Darlehen auf Wertpapiere........... & 2307244 & $\cdot$ & 0,1 & 2648189 & " & $0, \mathbf{I}$ & " \\
\hline 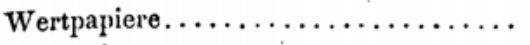 & 308406501 & n & $8,0 \cdot$ & 155459615 & n & 6,7 & $"$ \\
\hline Wechsel $\ldots \ldots \ldots \ldots \ldots \ldots \ldots \ldots \ldots \ldots$ & 8334 & " ( & $(0,0002) n$ & I 249432 & $*$ & $0, \mathrm{I}$ & $\cdot$ \\
\hline Sonstige Anlagen $\ldots \ldots \ldots \ldots \ldots \ldots$ & - & & - & - & & - & \\
\hline
\end{tabular}

Vergleicht man diese Zahlen mit denen des Vorjahrs, so erhält man folgendes Bild: Bei den Aktiengesellschaften haben zugenommen der Wert des Grundbesitzes um (; $949444 \mathscr{N}$, der Hypothekenbestand um $140486850 \mathscr{M}$, die Darlehen an öffentliche Körperschaften um 4279326 $\mathscr{N}$, die Darlehen auf Versicherungen um $29351035 \mathscr{M}$, die Darlehen auf Wertpapiere un $193316 \mathscr{A}$, die Wertpapiere um $136748154 \mathscr{M}$ (der Nennwert um rund 143 Millionen Mark); abgenommen hat dagegen der Bestand an Wechseln um $1966 \mathcal{M}$. Bei den Gegenseitigkeitsvereinen haben zugenommen die Darlehen an öffentliche Körperschaften um $2897011 \mathcal{M}$, die Darlehen auf Versicherungen um $13146432 \mathscr{N}$, die Wertpapiere um 52619254 $\mathscr{N}$ (der Nennwert um rund 54 Millionen Mark); abgenommen haben dagegen der Wert des Grundbesitzes um $2765928 \mathscr{N}$, der Hypothekenbestand um $114745052 \mathscr{M}$, die Darlehen auf Wertpapiere um $796931 \mathscr{N}$ und der Bestand an Wechseln um $644599 \mathscr{A}$. Mehr als im vorhergehenden Jahre haben allgemein zugenommen die Darlehen auf Versicherungen und die Wertpapiere (um 71 Millionen nach dem Nennwerte) infolge der Zeichnungen auf die deutsche Kriegsanleihe. Weniger als im Vorjahre haben zugenommen die Hypotheken (um 117 Millionen) und die Darlehen an öffentliche Körperschaften (um 34 Millionen). Dem Kapitalmarkte haben die allgemeinen deutschen Lebensversicherungsunternehmungen zugeführt: 4,18 Millionen Mark für Grundbesitz, 25,74 Millionen für Hypotheken, 7,18 Millionen für Darlehen an öffentliche Körperschaften, 189,37 Millionen für Wertpapiere, zusammen 226,47 Millionen gegenüber 308,72 Millionen im Vorjahre.

Die Prämienreserven und die Prämienüberträge sind in Tafel I 39 nach Versicherungsarten getrennt angegeben. Hierzu bedarf es indessen noch folgender Erläuterungen. Nach $\$ 58$ des Versicherungsaufsichtsgesetzes soll bei Rückversicherungen die Prämienreserve von dem rückversicherten Unternehmen berechnet, aufbewahrt und verwaltet werden. Die Rechnungsvorschriften bestimmen, daß als Prämienreserve in der Gewinnund Verlustrechnung und in der Bilanz nur die in eigener Verwaltung befindlichen Beträge geführt werden sollen. Für alle bis zum Inkrafttreten des Gesetzes abgeschlossenen Versicherungen sollen die Prämienreserven unter Berücksichtigung der.geltenden Rückversicherungsverträge eingestellt werden. Bei den nach dem Inkrafttreten des Gesetzes abgeschlossenen Versicherungen sollen die Prämienreserven für alle selbst abgeschlossenen Versicherungen einschließlich der in Rückdeckung gegebenen Summen unter dem Posten Prämienreserve nachgewiesen werden; für die in Rückdeckung übernommenen Versicherungen aber, soweit die Prämienreserven abzugeben sind, sollen diese Prämienreserven in der Gewinn- und Verlustrechnung bei den Vergütungen für in Rückdeckung übernommene Versicherungen verrechnet werden, also auch in der Bilanz nicht bei den Prämienreserven erscheinen. Die Gesellschaften verfahren bei der Behandlung 
der Prämienreserven nicht gleichmäßig. Ein Teil der Anstalten läßt die Prämienreserven in fremder Verwaltung ganz aus der Rechnung fort, während die übrigen Gesellschaften diese Posten bei anderen Stellen behandeln. Da der Vordruck L 3 der Rechnungsvorschriften die Mittel bietet, den Betrag der bei Rückversicherungen abgegebenen Rücklagen festzustellen, so sind diese Beträge in unsern Statistiken überall ausgeschieden. Dieses Verfahren entspricht nicht nur den Rechnungsvorschriften, sondern verhindert auch; daß die Rücklagen für solche Versicherungen, die von einer unter Reichsaufsicht stehenden Gesellschaft bei einer ebensolchen Gesellschaft in Rückdeckung gegeben sind, mehrfach aufgeführt werden.

Die Trennung der Prämienreserven und der Prämienüberträge erfolgt bei den verschiedenen Anstalten und bei verschiedenen Versicherungsarten nicht ñach denselben Grundsätzen, so daß die als Prämienreserven oder Prämienüberträge angegebenen Summen weder die reinen Prämienreserven noch die reinen Überträge darstellen. Prämienreserven und Prämienüberträge sind deshalb zusammengefaßt worden.

In Tafel I 39 sind für alle deutschen Aktiengesellschaften Prämienreserven und Prämienüberträge in eigener Verwaltung in Höhe von 3307,589 Millionen Mark nachgewiesen. Davon kommen 3200,398 Millionen Mark auf die Lebensversicherung und

\begin{tabular}{|c|c|c|c|c|c|c|}
\hline \multirow{3}{*}{ Versicherungsart } & \multicolumn{6}{|c|}{ Prämienreserven und Prämienüberträge } \\
\hline & \multicolumn{2}{|c|}{$\begin{array}{l}\text { bei der Gesamtheit } \\
\text { der Unternehmungen }\end{array}$} & \multicolumn{2}{|c|}{$\begin{array}{l}\text { bei den } \Lambda \text { ktien- } \\
\text { gesellschaften }\end{array}$} & \multicolumn{2}{|c|}{$\begin{array}{l}\text { bei den Gegen- } \\
\text { seitigkeitsvereinen }\end{array}$} \\
\hline & $1000 \mathscr{M}$ & $\%$ & $1000 \mathscr{M}$ & $\%$ & $1000 \mathscr{M}$ & $\%$ \\
\hline Versicherung auf den Todesfall .............. & 4643008 & $89, \mathrm{I}$ & 2797466 & 87,4 & I 845542 & 91,8 \\
\hline$n \quad n \quad$ Lebensfall .......... & 308255 & 5,9 & 233084 & 7,3 & 75171 & 3,8 \\
\hline Rentenversicherung $\ldots \ldots \ldots \ldots \ldots \ldots \ldots \ldots \ldots \ldots$ & 244512 & 4,7 & 157420 & 4,9 & 87092 & 4,3 \\
\hline Sonstige Versicherungen $\ldots \ldots \ldots \ldots \ldots \ldots \ldots$ & 14317 & 0.3 & 12428 & 0,4 & I 889 & 0.1 \\
\hline Zusammen $\ldots$ & 5210092 & 100,0 & 3200398 & 100,0 & 2009694 & $\overline{100,0}$ \\
\hline
\end{tabular}

107,191 Millionen Mark auf andere Versicherungszweige. Bei den Gegenseitigkeitsvereinen gehören die in der Tafel aufgeführten Beträge von zusammen 2009,694 Millionen Mark vollständig der Lebensversicherung an. Die gesamte Prämienreserve für Lebensund Krankenversicherung stellt sich danach für alle deutschen allgemeinen Unternehmungen auf 5 210,092 Millionen Mark, wovon 61,4 v. H. auf die Aktiengesellschaften und 38,6 v. H. auf die großen Gegenseitigkeitsvereine entfallen. In welchem Maße die einzelnen Versicherungsarten beteiligt sind, ist aus der Ubersicht auf S. $36^{*}$ zu entnehmen.

Die Verteilung des Gewinns ist in Tafel I 40 dargestellt. Wenn dabei die Summe des Gesamtgewinns der Gegenseitigkeitsvereine nicht mit der Summe der Jahresüberschüsse und der Vorträge aus dem Vorjahre, wie sie in der Bilanz (Tafel I 37) enthalten sind, übereinstimmt, so erklärt sich das daraus, daß auch die Verteilung des vom Allgemeinen Deutschen Versicherungsverein in Stuttgart aus dem Lebensversicherungsgeschäft erzielten Gewinns nachgewiesen ist, während die Bilanz dieses Vereins bei Gruppe II gegeben ist.

Sonach standen für die Gewinnverteilung zur Verfügung bei den Aktiengesellschaften $106008909 \mathscr{N}$ und bei den Gegenseitigkeitsvereinen $66830795 \mathscr{M}$; diese Beträge sind folgendermaßen verteilt worden: 


\begin{tabular}{|c|c|c|c|c|c|c|c|c|}
\hline \multirow{9}{*}{ 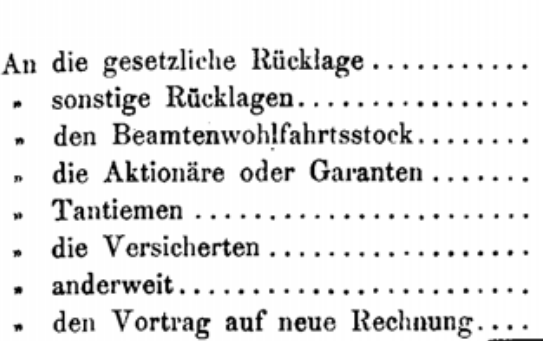 } & \multicolumn{4}{|c|}{27 Aktiengresellschaften } & \multicolumn{4}{|c|}{18 Gegenseitigkeitsvereine } \\
\hline & 162004 & $\mathscr{M}$ oder & 0,2 & & 480 I 48 & $\mathscr{M}$ oder & 0,7 & $\%$ \\
\hline & 8345480 & & 7,9 & . & 5563203 & & 8,3 & $n$ \\
\hline & 466095 & & 0,4 & • & 280699 & & 0,4 & $n$ \\
\hline & 7774499 & & 7,3 & n & 7174 & * & $(0,01)$ & $\cdot$ \\
\hline & 2602249 & & 2,5 & n & 173386 & n & 0,3 & $n$ \\
\hline & 84789040 & & 80,0 & - & 59484952 & n & 89,0 & $n$ \\
\hline & 674329 & & 0,6 & • & 821017 & & 1,2 & $n$ \\
\hline & I 195213 & $n$ & $\mathrm{I}, \mathrm{I}$ & $n$ & 20216 & & $0, \mathbf{I}$ & $n$ \\
\hline
\end{tabular}

Hinsichtlich der den Versicherten zugewiesenen Beträge ist zu beachten, daß einige Gesellschaften den den Versicherten zustehenden Gewinn in der Bilanz bereits verrechnet haben, so daß er hier nicht in die Erscheinung tritt. Diese Beträge (vgl. Fußnoten zu Tafel I 40 und IV 40) haben im Berichtsjahre insgesamt 5,769 Millionen Mark ausgemacht.

Die Bilanzen der Versicherungseinrichtungen von Berufsvereinigungen mit den zugehörigen Erläuterungen sind in den Tafeln I 42 bis I 46 gegeben. Die Gesamtbilanz schließt mit $144637913 \mathscr{M}$ ab; die Hauptposten weisen folgende Beträge auf:

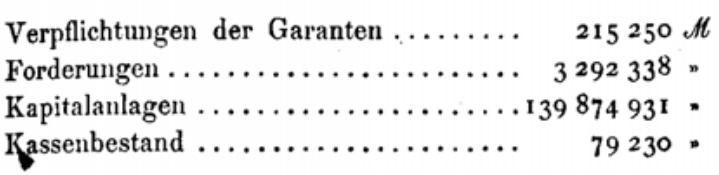

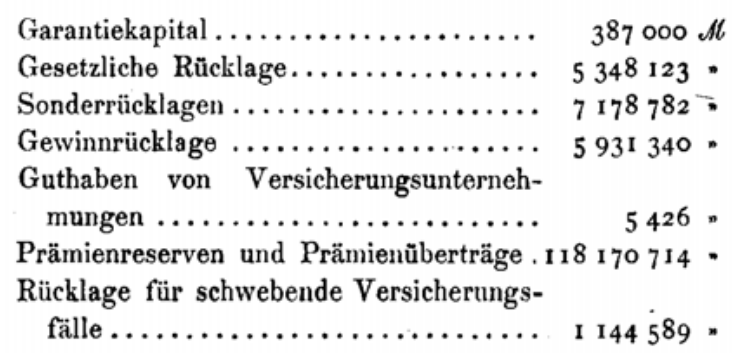

Von den insgesamt $139874931 \mathscr{M}$ betragenden Kapitalanlagen kamen $1229029 \mathscr{M}$ oder 0,9 v. H. auf Grundbesitz, $95569643 \mathscr{M}$ oder 68,3 v. H. auf Hypotheken, $8853260 \mathscr{M}$ oder 6,3 v. H. auf Darlehen an öffentliche Körperschaften, $31778636 \mathscr{M}$ oder $22,7 \mathrm{v}$. H. auf Wertpapiere und der Rest von $2444363 \mathscr{M}$ oder 1,8 v. H. auf Darlehen auf Versicherungen. Die Vergleichung des Bestandes am Ende des Vorjahrs mit dem Bestand am Ende des Berichtsjahrs ergibt eine Abnahme des Grundbesitzes um $12528 \mathscr{M}$ und eine Zunahme des Hypothekenbesitzes um $1962403 \mathscr{H}$, der Darlehen an öffentliche Körperschaften um $796781 \mathscr{M}$, des Buchwertes der Wertpapiere um $13986277 \mathscr{M}$ und der Darlehen auf Versicherungen um $216889 \mathscr{M}$.

Die Bilanzen von 12 Anstalten von Berufsvereinigungen weisen einen Gewinn von zusammen $1744303 \mathscr{M}$ auf, der wie folgt verteilt worden ist: $103456 \mathscr{M}$ oder 5,9 v. $\mathrm{H}$. an die gesetzliche Rücklage, $417346 \mathscr{M}$ oder 23,9 v. H. an die Sonderrücklagen, $6073 \mathscr{M}$ oder 0,3 v. $H$. an den Beamtenwohlfahrtsstock, $5138 \mathscr{M}$ oder 0,3 v. H. Tantiemen, $1063402 \mathscr{M}$ oder 61,0 v. H. an die Versicherten, $22022 \mathscr{N}$ oder 1,3 v. H. Vortrag auf neue Rechnung und $125800 \mathscr{M}$ oder 7,2 v. H. für anderweite Zwecke.

Die Bilanzen von 17 ausländischen Gesellschaften, deren Lebensversicherungsgeschäfte in dieser Statistik behandelt sind, finden sich in Tafel I 47. Die Bilanzen sind nach Möglichkeit den für die Bilanzen der deutschen Unternehmungen geltenden Grundsätzen angepaßt worden. 
Von. besonderem Interesse ist die Art der Kapitalanlagen (Tafel I 48), die von den Gesellschaften der verschiedenen Länder, sei es aus eigenem Antriebe, sei es auf Veranlassung der Aufsichtsbehörden, bevorzugt wird; deshalb sind in der folgenden Ubersicht die in der Tafel I 48 aufgeführten ausländischen Gesellschaften nach Heimatländern zusammengefaßt und zum Vergleiche die Verhältniszahlen für die deutschen Unternehmungen wiederholt:

Von je $1000 \mathscr{N}$ Kapitalanlagen entfielen auf:

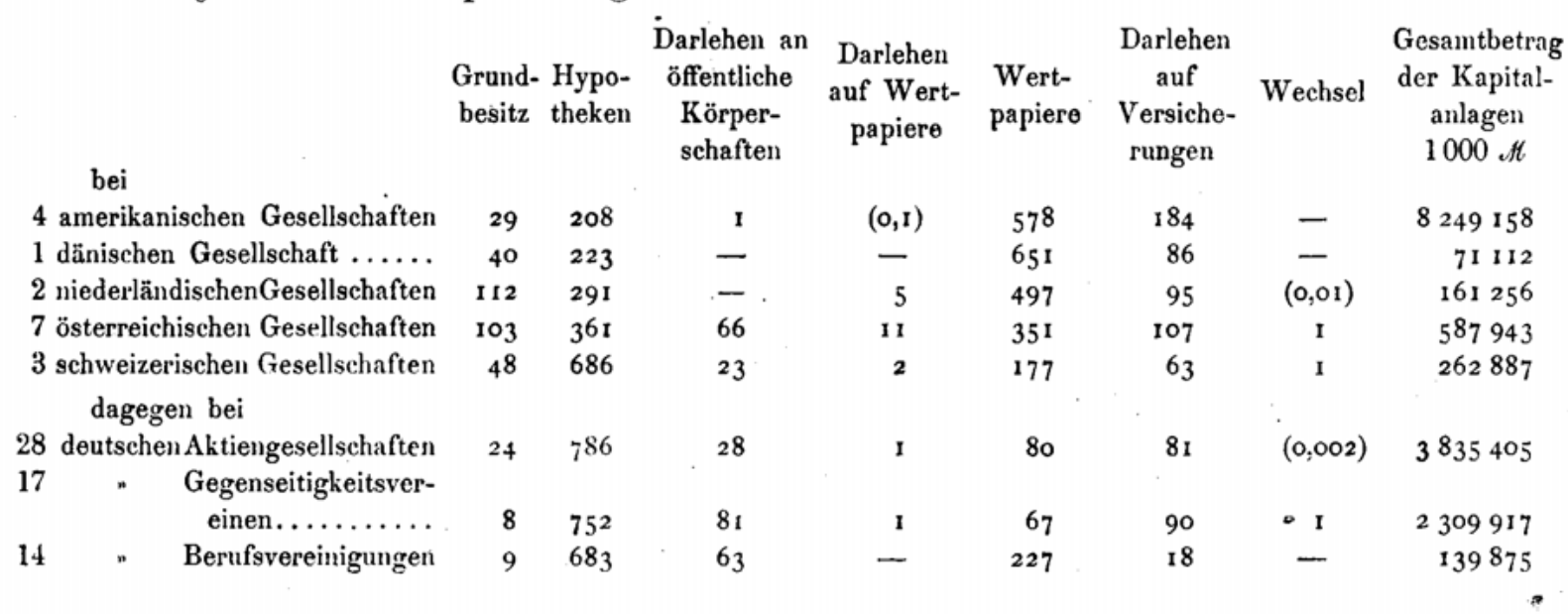

Das aus. landische Geschaft der deutschen Unternehmungen
Von den allgemeinen deutschen Unternehmungen haben die Kapitalversicherung in den wichtigeren Staaten des Auslandes mittelbar oder unmittelbar betrieben: 24 in der Schweiz, 22 in Österreich, 21 in Dänemark, 19 in Belgien, 17 in den Niederlanden, je 8 in Frankreich und Norwegen, je 7 in Schweden und in der Türkei, je 6 in Ägypten, Luxemburg, Rußland und Spanien, 5 in Ungarn, je 4 in China, England, Finnland, Portugal, Rumänien und in den Vereinigten Staaten von Amerika, je 3 in Argentinien und in Brasilien, 2 in Bolivien, je 1 in Bulgarien, Chile, Griechenland und in Japan. In der Rentenversicherung ist das Geschäft natürlich auch im Auslande weniger verbreitet; es haben gearbeitet: 12 Unternehmungen in der Schweiz, 11 in Belgien, 9 in den Niederlanden, 8 in Österreich, 7 in Dänemark, je 5 in Frankreich und in Luxemburg, 4 in Rußland, je 3 in England, Japan, Norwegen, Rumänien, Schweden, Türkei und in den Vereinigten Staaten von Amerika, je 2 in Ägypten, Brasilien, Spanien und Ungarn, je 1 in Argentinien, Chile, China, Finnland, Griechenland, Mexiko und Portugal.

In der Kapitalversicherung hatten die Unternelımungen zusammen Ende 19 I 5 im Auslande 300590 laufende Verträge über 1551,762 Millionen Mark, das sind 2,3 v. H. aller Versicherungen und 9,7 v. H. der gesamten Versicherungssummen; die vereinnahmten Beiträge, einschließlich der für Rückversicherungen wieder abgegebenen Beiträge betrugen 67,403 Millionen Mark, wovon 64,938 Millionen oder 96,3 v. H. für selbst abgeschlossene und 2,465 Millionen oder 3,7 v. H. für in Rückdeckung übernommene Versicherungen zu rechnen sind. An Versicherungsleistungen wurden 33,481 Millionen Mark gewährt, nämlich 30,987 Millionen für selbst abgeschlossene Versicherungen und 2,494 Millionen für Rückversicherungen; von den in der ausländischen Kapitalversicherung überhaupt fällig gewordenen Beträgen kommen demnach 7,4 v. H. auf die in Rückdeckung übernommenen Versicherungen. 
In der Rentenversicherung liefen Ende 1915 im Auslande 3964 Versicherungen über 1,776 Millionen Mark Jahresrente, das sind 5,4 v. H. aller Versicherungsscheine und 5,7 v. H. der gesamten Jahresrente. An Beiträgen wurden vereinnahmt 0,229 Millionẹ Mark. Für Versicherungsleistungen waren 1,157 Millionen Mark bereitzustellen.

Zu einer eingehenden Vergleichung des ausländischen Geschäfts aller deutschen Lebensversicherungs-Unternehmungen mit dem deutschen Geschäfte der ausländischen Gesellschaften fehlt der Stoff, doch bieten folgende Zahlen wenigstens einige Anlialtspunkte:

Im Jahre I 9 I 5 hat die Beitragseinnahme betragen:

\begin{tabular}{|c|c|c|c|}
\hline & $\begin{array}{l}\text { insgesamt } \\
1000 \varkappa\end{array}$ & $\begin{array}{l}\text { für selbst ab- } \\
\text { geschlossene } \\
\text { Versicherungen } \\
1000 \AA\end{array}$ & $\begin{array}{l}\text { für in Rückdeckung } \\
\text { übernommene } \\
\text { Versicherungen } \\
1000 \mathscr{H}\end{array}$ \\
\hline $\begin{array}{l}\text { bei den deutschen Gesellschaften aus dem aps- } \\
\text { ländischen Geschäfte } \ldots \ldots \ldots \ldots \ldots \ldots \ldots \ldots \ldots \ldots \ldots \text {. } \\
\text { bei den ausländischen Gesellschaften aus den }\end{array}$ & 67632 & 65167 &.$^{2465}$ \\
\hline deutschen Geschäfte..$\ldots \ldots \ldots \ldots \ldots \ldots$ & 45424 & $445^{88}$ & 836 \\
\hline
\end{tabular}

Hiernach haben insgesamt die deutschen Gesellschaften für Lebensversicherungen 22,208 Millionen Mark mehr an Beiträgen aus dem Auslande als die hier berücksichtigten ausländischen Gesellschaften aus dem Deutschen Reiche eingenommen. Die Zahlungen an Versicherungsleistungen haben betragen:

\begin{tabular}{|c|c|c|c|}
\hline & insgesamt & $\begin{array}{l}\text { für selbst ab- } \\
\text { geschlossene } \\
\text { Versicherungen }\end{array}$ & $\begin{array}{c}\text { für in Rückdeckung } \\
\text { übernommene } \\
\text { Verșicherungen }\end{array}$ \\
\hline bei den deutschen Gesellschaften für ausländische & $1000 \mathscr{M}$ & $1000 \mu$ & $1000 \mu$ \\
\hline $\begin{array}{l}\text { Versicherungen } \ldots \ldots \ldots \ldots \ldots \ldots \ldots \ldots \ldots \ldots \ldots \ldots \ldots \ldots \ldots \ldots \\
\text { bei den ausländischen Gesellschaften für deutsche }\end{array}$ & 34638 & 32144 & 2494 \\
\hline Versicherungen $\ldots \ldots \ldots \ldots \ldots \ldots \ldots \ldots \ldots$ & 39350 & 38605 & 745 \\
\hline
\end{tabular}

Näheres hierüber findet man in der "Entwickelung " S. 13.

Außer den besprochenen und in den Tafeln behandelten deutschen Versicherungsunternehmungen steht, wie bereits erwähnt, gemäß $\S 2$ V.A.G. noch eine Anzahl kleinèr Sterbe- und Pensionskassen unter Reichsaufsicht, die für eng begrenzte Kreise Die kleinen Pensionsund Sterbe bestimmt und vielfach von gewerblichen Firmen nur für die eigenen Angestellten oder Arbeiter eingerichtet sind. Die Zahl dieser Gegenseitigkeitsvereine ist schwankend; einerseits sind diese Unternehmungen wohl kaum sämtlich bekannt, auch entstehen stets neue derartige Vereine; anderseits geht die Beaufsichtigung nicht selten gemäß $\S 3$ Abs. 2 nachträglich an die Landesbehörde über. Andere Vereine verfallen der Auflösung. Wie dem auch sei, die Zahl der unter Reichsaufsicht stehenden Sterbekassen und Pensionskassen wird immer nur sehr klein sein im Vergleiche zu der Zahl der unter Landesaufsicht befindlichen; wenn hier einige Mitteilungen über die unter unserer Aufsicht stehenden Kassen gemacht werden, so können daraus keineswegs Schlüsse über die Bedeutung des Sterbekassen-und Pensionskassenwesens im Reiche gezogen werden.

Für das Jahr I9I5 haben insgesamt 133. solcher Sterbekassen dem Aufsichtsamte befriedigende Jahresberichte eingereicht. Danach ist die Zahl der Mitglieder Ende 1915 auf rund 523858 anzusetzen; die Einnahmen haben im Berichtsjahre $9172514 \mathscr{M}$ betragen, die Ausgáben $5499710 \mathscr{M}$. 
Von den Einnahmen kommen $6959955 \mathscr{M}$ oder 75,9 v. H. auf Beiträge für die Mitglieder, $25937 \mathscr{M}$ oder 0,3 v. H. auf Eintrittsgelder, $1999442 \mathscr{M}$ oder 21,8 v. H. auf Vermögenserträge und $187180 \mathscr{M}$ oder 2,0 v. H. auf andere Einnahmen. Von den Ausgaben kommen $3430542 \mathscr{M}$ oder $62,3 \mathrm{v}$. H. auf Sterbegelder, $884911 \mathscr{N}$ oder 16,0 v. H. auf Krankengelder, $43430 \mathscr{M}$ oder 1,0 v. H. auf Zahlungen für vorzeitig

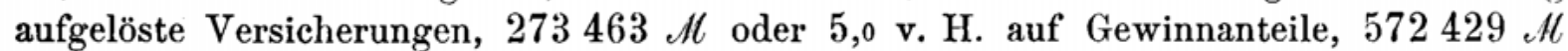
oder 10,4 v. H. auf Verwaltungskosten und Steuern und $294935 \mathscr{M}$ oder 5,3 v. H. auf die übrigen Ausgaben. Das Vermögen dieser Kassen belief sich am Schlusse des Jahres I9I5 auf $50499933 \mu$.

Die Zahl der Pensionskassen der genannten Art, die in befriedigender Weise über ihren Geschäftsbetrieb im Jahre 1915 an das Amt berichtet haben, betrug 87; diese Kassen hatten Ende I9I4 114154 aktive Mitglieder und an Bezugsberechtigten 5701 Pensionierte, 3051 Witwen und 1401 Waisen und Ende 1915 113013 Aktive, 5826 Pensionierte, 3354 Witwen und 1555 Waisen. Die Einnahmen betrugen im Berichtsjăhre $28926410 \mathscr{M}$, während $10691918 \mathscr{M}$ verausgabt wurden. Von den Einnahmen entfallen $17240565 \mathscr{M}$ oder 59,6 v. H. auf Beiträge für die Mitglieder, $171004 \mathscr{M}$ oder $0,6 \mathrm{v} . \mathrm{H}$. auf Eintrittsgelder, $9025425 \mathscr{M}$ oder 31,2 v. H. auf Vermögenserträge und $2489416 \mathscr{M}$ oder 8,6 v. $\mathrm{H}$. auf anderweitige Einnahmen. Von den Ausgaben entfallen 5802626 , $H$ oder 54,2 v. H. auf Pensionen, $80795 \mathscr{M}$ oder 1,0 v. H. auf Krankengeld, $431390 \mathscr{M}$ orler 4,0 v. H. auf Sterbegeld und sonstige Leistungen an Versicherte, 900814 th oder 8,4 v. H. auf Abfindungen beim Austritt aus der Kasse (meist infolge Wechsels der Dienststelle), 63 $798 \mathscr{M}$ oder 0,6 v. H. auf Gewinnanteile, $344363 \mathscr{M}$ oder 3,3 v. H. auf Verwaltungskosten und Steuern und $3068132 \mathscr{M}$ oder 28,6 v. $\mathrm{H}$. auf die übrigen Ausgaben. Der niedrige Satz für Verwaltungskosten hat seinen Grund in erster Linie darin, daß bei den von gewerblichen Firmen eingerichteten Versicherungskassen die Verwaltungskosten meistens vollständig

von den Firmen übernommen werden und somit die Kassen nicht belasten. Das Vermögen dieser Pensionskassen belief sich am Ende des Berichtsjahrs auf $216141014 \mathscr{M}$.

\section{Gruppe II, Unfall- und Haftpflichtversicherung}

Die Unternehmungen
Von den großen deutschen Versicherungsunternehmungen, welche der Reichsaufsicht unterstehen, waren im Jahre I 9 I 532 auf dem Gebiete der Unfall- und Haftpflichtversicherung tätig, 31 Aktiengesellschaften und 1 Versicherungsverein auf Gegenseitigkeit. Die Anzall der im Berichtsjahre zu behandelnden Gesellschaften hat sich um zwei vermehrt, indem die Berlinische Lebens-Versicherungs-Gesellschaft in Berlin und der Globus, Versicherungs-Aktien-Gesellschaft in Hamburg, die jetzt auch die Unfall- und die Haftpflichtversicherung betreiben, hinzugekommen sind, während die Freia, BremenBerliner Versicherungs-Aktiengesellschaft in Berlin, infolge Vereinigung mit dem Globus, Versicherungs-Aktien-Gesellschaft in Hamburg, und der Nordstern, Feuer-VersicherungsAktien-Gesellschaft in Berlin-Schöneberg, infolge Rückversicherung des Geschäfts in seiner Gesamtheit bei dem Nordstern, Unfall- und Haftpflicht-Versicherungs-AktienGesellschaft in Berlin-Schöneberg, fortgelässen sind. 
Außerdem berichtet die vorliegende Statistik wieder über acht ausländische Gesellschaften, dieselben, die in der vorjährigen Statistik behandelt sind.

Zur Beurteilung des Umfanges des Geschäfts der deutschen Gesellschaften soll die Tafel II 1 dienen. Daraus ist $\mathrm{zu}$ ersehen, daß bei diesen Unternehmungen am Ende des Jahres I 9 I 5 in der Unfallversicherung 1215808 und in der Haftpflichtversicherung 2724925 Versicherungsscheine über selbst abgeschlossene oder in Rückdeckung übernommene Versicherungen in Kraft waren.

In der Haftpflichtversicherung wurden Versicherungen mit Gewinnbeteiligung außer von dem Allgemeinen Deutschen Versicherungs-Verein in Stuttgart nur von der Oberrheinischen Versicherungs-Gesellschaft in Mannheim, und zwar nur in ganz geringem Umfang abgeschlossen. Auch in der Unfallversicherung kommen Versicherungen mit Gewinnbeteiligung nur bei wenigen Unternehmungen in Betracht. Von den Aktiengesellschaften haben nur zwei solche Versicherungen abgeschlossen, darunter eine auch nur in ganz geringem Umfange. Von der Beitragseinnahme des Geschäftsjahrs in Höhe von $34590408 \mathscr{A t}$, für selbst abgeschlossene Unfallversicherungen sämtlicher Unternehmungen kamen aufVersicherungen mit Gewinnbeteiligung nur $10192128 \mathscr{M}$ oder 29,5 v. H., oder, wenn man den Allgemeinen Deutschen Versicherungs-Verein in Stuttgart, weil er eine Gewinnbeteiligung nur auf Grund seines Charakters als Gegenseitigkeitsverein kennt, ausscheidet, 17,6 v. H.

In den für die Unfallversicherung gegebenen Zahlen ist auch die Zeitungsabonnentenversicherung enthalten, die von 2 Gesellschaften nämlich der Deutschland, Lebensversicherungs-Aktien-Gesellschaft zu Berlin, und der Nürnberger LebensversicherungsBank betrieben wird. Die Anzahl der hierdurch versicherten Personen beläuft sich auf rund 2344000 mit $2353000 \mathscr{N}$ Beitragseinnahme.

Für das Rückversicherungsgeschäft lassen sich folgende Zahlen angeben. In der Unfallversicherung waren in Rückdeckung übernommen für $2504318 \mathscr{M}$ Beiträge oder 6,8 v. H. der ganzen Beitragseinnahme von $37094726 \mathscr{N}$ und in Rückdeckung gegeben für $7073126 \mathscr{M}$ oder für 19,1 v. H. der ganzen Beitragseinnahme. In der Haftpflichtversicherung waren in Rückdeckung übernommen für $2073971 \mathscr{N}$ Beiträge oder 4,3 v. H. und in Rückdeckung gegeben für $11235058 \mathscr{M}$ oder 23,2 v. H. der gesamten Beitragseinnahme von $48465262 \mathcal{M}$.

In diesen wie in allen folgenden Zahlen sind beim Allgemeinen Deutschen Versicherungs-Verein in Stuttgart in der Haftpflichtversicherung auch die auf die Versicherung gegen Wasserleitungsschäden und einige andere kleine Sachversicherungszweige bezüglichen Zahlen enthalten.

Vergleicht man den Versicherungsbestand am Ende des Berichtsjahrs mit dem entsprechenden Bestand am Ende des Vorjahrs (Tafel II 2), so findet man, daß die Vergleich mit dem Vo Zahl der Versicherungsscheine bei der Unfallversicherung um 213657 oder 14,9 v. H. und bei der Haftpflichtversicherung um 63214 oder 2,3 v. H. des Bestandes am Ende des Vorjahrs abgenommen hat. Die gesamte Beitragseinnahme hat im Geschäftsjahre bei der Unfallversicherung $12320030 \mathscr{M}$ oder 24,9 v. H. und bei der Haftpflichtversicherung $9441803, \mathscr{M}$ oder $16,3 \mathrm{v}$. H. weniger als im Vorjahre betragen. 
Dieser weitere erhebliche Rückgang in der Beitragseimnahme ist lediglich eine Folge des Krieges. In der Friedenszeit hatte die Einnahme von Jahr zu Jahr zugenommen und selbst die mit der Durchführung der weitgelienden Tarifreform vom Jahre 1904 verbundenen Schwierigkeiten im Geschäftsbetriebe hatten 1905 wohl ein Stocken im Anwachsen, aber doch keinen Rückgang der Beitragseinnahme zur Folge.

Dasselbe gilt auch für die Zahlungen für Schadenfälle des Geschäftsjahrs.

Die

Betritebs-

Vinnahmen

und

Ausgaben

des

Berichts-

jahrs

In unseren Statistiken sind von vornherein die den einzelnen Versicherungszweigen eigentümlichen und in den Büchern getrennt behandelten Posten einzeln aufgeführt worden; seit 1905 konnten auch die allgemeinen Verwaltungskosten und die Steuern mit befriedigender Genauigkeit nach Geschäftszweigen getrennt werden. Seitdem ist der Gewinn oder Verlust aus dem eigentlichen Versicherungsbetriebe des Geschäftsjahrs bei jedem Zweige ermittelt worden; Kinnahmen und Ausgaben, die nicht unmittelbar mit dem Versicherungsbetriebe des Berichtsjahrs zusammenhängen, wie z. B. bei der Sachversicherung die Erträge der Vermögensverwaltung, bleiben dabei außer Betracht (vgl. "Entwickelung " S. 33). Bei der Unfall- und Haftpflichtversicherung ist indessen der Betriebs-Gewinn oder -Verlust nicht vollständig ermittelt worden, weil der Ertrag der die Prämienreserve deckenden Kapitalanlagen, der zweifellos zu den Betriebs-Einnahmen zu rechnen ist, nicht sicher angegeben werden kann. Schätzungen des Amts sollen aber in dieser Statistik vermieden werden. Um alle Mißverständnisse auszuschließen, ist in Tafel II 3 kein Gewinn oder Verlust eingestellt. Das Ergebnis der Vermögensverwaltung (vgl. S. 44*) findet sich in Tafel II 5. Der Gewinn oder Verlust des alle Zweige umfassenden Gesamtgeschäfts wird in der Bilanz ausgewiesen.

Als Betriebs-Einnahmen sind bei Gruppe II verrechnet die Beiträge für das Geschäftsjahr, d.h. die Beiträge unter Berücksichtigung der Uberträge aus dem Vorjahr und der Uberträge auf das folgende Jahr, einschließlich der an die Rückversicherungsgesellschaften gezahlten Rückversicherungsbeiträge, ferner die Ausfertigungsgebühren, die Vergütungen der Rückversicherer, der Unterschied zwischen der Schadenrücklage aus dem Vorjahr und den Zahlungen und Rückstellungen für Versicherungsfälle der Vorjahre aus selbst abgeschlossenen Versicherungen, sofern die Schadenrücklage größer ist, und der etwaige Mehrbetrag der aus dem Vorjahr übernommenen Prämienreserve gegenüber der am Schlusse des Jahres zu stellenden Prämienreserve. Die Betriebs-Ausgaben setzen sich bei Gruppe II zusammen aus den Zahlungen für die selbst abgeschlossenen und für die in Rückdeckung übernommenen Versicherungen einschließlich des Anteils der Rückversicherer, aus den Rückversicherungsbeiträgen, dem Unterschiede zwischen der Schadenrücklage, aus dem Vorjahr und den Zahlungen und Rückstellungen für Versicherungsfalle der Vorjahre aus selbst abgeschlossenen Versicherungen, sofern die Schadenrücklage kleiner ist, und endlich aus der Zunahme der Prämienreserve. Es soll hier aber besonders betont werden, daß der Unterschied zwischen der Schadenrücklage aus dem Vorjahr und den Zahlungen und Rückstellungen für Versicherungsfälle aus selbst abgeschlossenen Versicherungen der Vorjahre allein keinen Schluß darüber zuläßt, ob die Schadenrücklage zulänglich bęmessen war. Denn einerseits sind aus der Schadenrücklage auch andere Leistungen als Zahlungen für Versicherungsfälle der Vorjahre zu bewirken, z. B. Zahlungen für die in Rückdeckung übernommenen Versicherungen und Zuführungen zur Prämienreserve. Anderseits wird die Schadenrücklage meist ohne den Anteil der Rückversicherer eingestellt, 
wogegen die Zahlungen einschließlich dieses Anteils erscheinen. Auch enthalten die Zahlungen für Versicherungsfälle der Vorjahre Beträge, die nicht aus der Schadenrücklage, sondern aus der Prämienreserve zu decken sind.

Die Betriebs-Einnahmen des Berichtsjahrs haben ohne die Erträge der Prämienreserven insgesamt bei den 32 deutschen Gesellschaften $112449653 \mathscr{M}$ betragen; davon kamen auf Beiträge für Unfallversicherung $37926391 \mathscr{M}$ oder 33,7 v. H., auf Beiträge für Haftptlichtversicherung 48927784 , $/$ oder 43,5 v. H. und auf Vergütungen der Rückversicherer 16416712 , $h$ oder 14,6 v. H. aller dieser Betriebs-Einnahmen.

Die Summe der Betriebs-Ausgaben ohne Verwaltungskosten beziffert sich auf $71600047 \mathscr{N}$; davon entfallen $41889089 \mathscr{M}$ auf Zahlungen für Versicherungsfälle im Geschäftsjahr aus selbst abgeschlossenen Versicherungen, und zwar (Tafel II 4) $15575481 \mathscr{N}$ oder 21,8 v. H. aller Betriebs-Ausgaben auf Unfallversicherungsfälle, ferner $21082026 \mathscr{M}$ oder 29,4 v. H. auf Haftpflichtversicherungsfälle, $1887600 \mathscr{M}$ oder 2,6 v. H. auf laufende Renten und endlich $3343982 \mathscr{M}$ oder 4,7 v. H. auf Beitragsrückgewährbeträge. Von der Summe der Betriebs-Ausgaben entfallen ferner $7003609 \mathscr{H}$ oder 9,8 v. $H$. auf Vergütungen für die in Rückdeckung übernommenen Versicherungen (darunter $2384818 \mathscr{N}$ oder 3,3 v. $H$. für Unfallversicherungsfälle und $3050836 \mathscr{M}$ oder 4,3 v. H. für Haftpflichtversicherungsfälle), $7073126 \mathscr{M}$ oder 9,9 v. $H$. auf Rückversicherungsbeiträge für Unfallversicherungen und $11235056 \mathscr{M}$ oder 15,7 v. H. auf Rückversicherungsbeiträge für Haftplichtversicherungen.

Die 32 Gesellschaften haben an Verwaltungskosten und Steuern insgesamt $30089056 \mathscr{M}$ für das Unfall- und Haftpflichtversicherungsgeschãft aufgewandt.

Wenn man annimmt; daß die Vergütungen für die in Rückdeckung übernommenen Versicherungen sich nur auf Versicherungsfälle des Geschäftsjahrs beziehen und davon absieht, daß ein Teil der laufenden Renten, die ja in ihrer Gesamtheit nur geringe Beträge erfordern, aus früheren Jahren herrührt, so kann man den Gesamtbetrag der Schadenzahlungen (49407733 $\mathscr{M}$ ) in Beziehung setzen zur Beitragseinnahme für das Geschäftsjahr (86854175 M). Man findet dann, daß von je $1000 \mathscr{M}$ Beitragseinnahme $569 \mathscr{M}$ für Schadenzahlungen verausgabt wurden; weitere $31 \mathscr{M}$ wurden zur Erhöhung der Prämienreserven verwandt. 'Tatsächlich ist allerdings für eigentliche Schäden etwas weniger aufgewandt worden; denn einzelne Gesellschaften verrechnen bei den Schadenzahlen auch noch mancherlei andere Beträge; z. B. Schadenermittelungskosten. - In den letzten fünf Jahren haben diese Zahlen betragen:

\begin{tabular}{|c|c|c|c|c|c|}
\hline & 1911 & 1912 & 1913 & 1914 & 1915 \\
\hline bei den $Z$ uführungen zur Prämienmeserve. & 515 ett & $\begin{array}{r}496 \mu \\
39 .\end{array}$ & $526 \mathscr{N}$ & $519 \mathscr{N}$ & $\begin{array}{r}569 \mathscr{N} \\
31 \text { n }\end{array}$ \\
\hline
\end{tabular}

Das Ergebnis der Vermögensverwaltung wird von den Unternehmungen in der Regel nicht nach den einzelnen Geschäftszweigen getrennt; nur der Lebensversicherung werden immer bestimmte Erträge überwiesen, weil das der Betrieb dieses Geschäfts erfordert. Es besteht keine Veranlassung, in dieser Statistik von dem Verfahren der Gesellschaften abzugehen. Soweit also nicht ein Teil des Ertrags einem bestimmten Zweige zugewiesen ist, wird das Ergebnis zumeist nur insgesamt aufgeführt und bei derjenigen Gruppe mitgeteilt, zu der das Hauptgeschäft der Unternehmung gehört. 
Im einzelnen geben die Fußnoten der Tafeln überall da Auskunft, wo nur ein Teil der Vermögensverwaltung in Betracht genommen worden ist.

Unter den Erträgen stehen wie überall so auch hier die Zinsen im Vordergrund, nämlich mit $11978158 \mathscr{N}$; der Kursgewinn bleibt um $811780 \mathscr{N}$ hinter dem Kursverluste zurück, der sonstige Gewinn beträgt $521374 \mathscr{M}$, der sonstige Verlust $35834 \mathscr{M}$. Als reiner Ertrag sind $12263032 \mathscr{N}$ zu verzeichnen.

Eine Bilanz ist in dieser Statistik grundsätzlich nur für das Gesamtgeschäft einer jeden Gesellschaft gegeben und bei derjenigen Gruppe aufgeführt, zu der das Unternehmen seinem Hauptbetriebe nach - Transportversicherung immer ausgeschlossen gehört. So bleiben bei Gruppe II von den 32 deutschen Gesellschaften, welche Unfall- oder Haftpflichtversicherungen unmittelbar abschließen, nur 8 Anstalten übrig. Die Bilanzen der Berlinischen Lebens-Versicherungs-Gesellschaft, von Deutschland, Victoria, Urania, Providentia, Janus, Teutonia, Atlas, Magdeburger Lebens-Versicherungs-Gesellschaft, Wilhelma, Bayerische Versicherungs-Bank, Nürnberger Lebensversicherungs-Bank und Germania finden sich bei Gruppe I (Tafel I 37), die Bilanzen von Victoria Feuer-Versicherungs-Aktien-Gesellschaft, Vaterländische und Rhenania, Thuringia, Iduna F.U.H. u. R.V.A.G., Albingia, Globus, Hamburg-Bremer, Rheinland, Preußische National V.G. und Stuttgart-Berliner V.A.G. bei Gruppe IV ('Tafel IV 38) und von Hammonia bei Gruppe V (Tafel V 9).

^us den in Tafel II 6 für die 8 Gesellschaften gegebenen Bilanzen mögen hier dıe folgenden Schlußzahlen Platz finden:
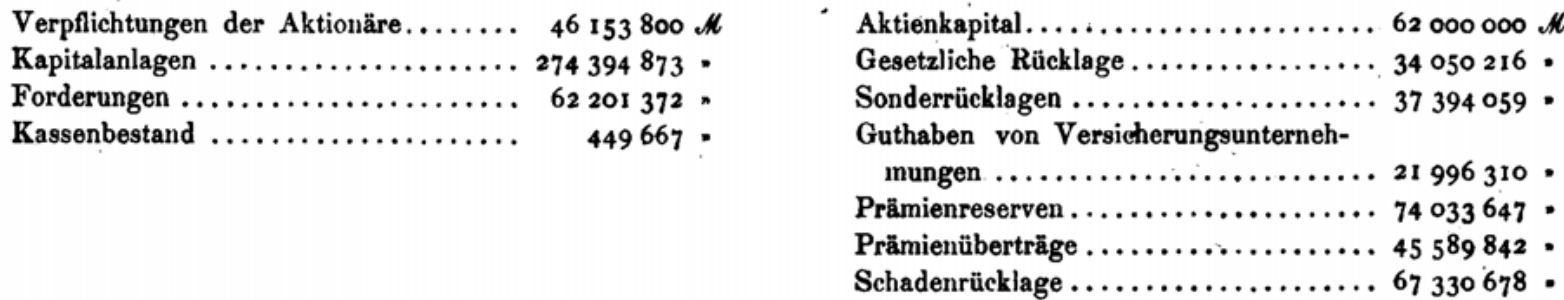

Dabei ist zu erwähnen, daß aus den Nebenbetrieben der Gesellschaften von den Prämienreserven 57175557 $\mathscr{M}$ auf Lebensversicherungen und von den Prämienüberträgen $25370889 \mathscr{M}$ auf nicht zur Gruppe II gehörige Versicherungszweige entfallen; für die Unfall- und Haftpflichtversicherung bleiben danach an Prämienreserven nur $16858090 \mathscr{M}$ und an Uberträgen $20218953 \mathcal{M}$.

Die Kapitalanlagen $(274394873 \mathscr{M})$ verteilen sich auf folgende Wertarten: $24126293 \mathscr{M}$ oder 8,8 v. H. Grundbesitz, $142961796 \mathscr{N}$ oder 52,1 v. H. Hypotheken, $28822543 \mathscr{M}$ oder 10,5 v. H. Darlehen an öffentliche Körperschaften, $573932 \mathscr{M}$ oder 0,2 v. H. auf Darlehen auf Wertpapiere, $73936600 \mathscr{N}$ oder 27,0 v. H. Wertpapiere, $3859838 \mathscr{M}$ oder 1,4 v. H. Darlehen auf Versicherungen und $113871 \mathscr{M}$ oder 0,04 v. H. Wechsel: Stellt man diese Zahlen denen des Vorjahrs gegenüber, so haben die Darlehen auf Wertpapiere um $299897 \mathscr{M}$, die Wertpapiere um $17561615 \mathscr{M}$ (nach dem Nennwert um $18125523 \mathscr{M}$ ), die Darlehen auf Versicherungen um $410665 \mathscr{N}$, die Wechsel um 46705 $\mathscr{M}$ zugenommen; dagegen haben der Grundbesitz um $340638 \mathscr{M}$, der Besitz an Hypotheken um $860163 \mathscr{M}$ und die Darlehen an öffentliche Körperschaften um $121400 \mathscr{M}$ abgenommen. Sieht man von den Darlehen auf Versicherungen, die den 
öffentlichen Geldverkehr nicht unmittelbar berühren, ab, so haben jene 8 Gesellschaften dem Kapitalmarkte $16586016 \mathscr{M}$ zugeführt.

Jede der 8 Gesellschaften hat mit einem Gewinn abgeschlossen, der sich für alle Gesellschaften zusammen nach der Bilanz auf $19213020 \mathscr{H}$ und mit Einschluß des Gewinnvortrags aus dem Vorjahr auf $21397650 \mathscr{M}$ stellt gegen $18262834 \mathscr{M}$ und $20531704 \mathscr{M}$ im Vorjahre. Von dem Gewinne des Berichtsjahrs entfallen auf die Lebensversicherungsabteilung des Allgemeinen Deutschen Versicherungs-Vereins in Stuttgart $3260327 \mathscr{M}$, dessen Verteilung aus Tafel I $40 \mathrm{zu}$ ersehen ist. Von dem Reste, $18137323 \mathscr{H}$, sind nach Tafel II 8 überwiesen $6860 \mathscr{M}(0,04 \mathrm{v}$. H.) an die gesetzliche Rücklage, $685042 \mathcal{M}(3,8$ v. H.) an sonstige Rücklagen, $412015 \mathscr{M}(2,3$ v. H.) an den Beamtenwohlfahrtsstock, $5987348 \mathscr{N}(33,0$ v. H.) an die Aktionäre; ferner sind zugeteilt $1575364 \mathscr{M}(8,7$ v. H.) an Tantiemen, $5916607 \mathscr{M}(32,6$ v. H.) Gewinnanteile an die Versicherten sowie $1295984 \mathscr{M}(7,1$ v. H.) anderweit; endlich sind $2258103 \mathscr{M}$ $(12,5$ v. H.) auf neue Rechnung vorgetragen.

In der diesjährigen Bilanz stehen die gesetzlichen Rücklagen um $6152 \mathscr{N}$ und die Sonderrücklagen um $1946911 \mathscr{M}$ höher als in der Bilanz für Ende I914.

Von den hier behandelten 32 deutschen Unternehmungen haben 17 Anstalten auch mehr oder weniger umfangreiche Unfall- und Haftpflichtversicherungsgeschäfte im Auslandc abgeschlossen, nämlich in Finnland, Japan, den Vereinigten Staaten von Amerika und Bulgarien je 1, in Portugal, Rụmänien und Rußland je 2, in Italien, Spanien, der 'Türkei und Ungarn je 3, in England und Frankreich je 4, in Norwegen 5, in Luxemburg und Schweden je 6, in Belgien und Dänemark je 10, in der Schweiz 11, in Österreich 12 und endlich in den Niederlanden 13.

Diese Unternehmungen hatten im Auslande 156464 Versicherungen in Kraft, das sind 4,0 v. H. aller ihrer Versicherungen. Die vereinnahmten Beiträge, einschließlich der für Rückversicherungen wieder abgegebenen Teile, betrugen für das ausländische Geschäft insgesamt 7,188 Millionen Mark, wovon 6,237 Millionen Mark für die selbst abgeschlossenen und 0,95t Millionen Mark für die in Rückdeckung übernommenen Versicherungen zu rechnen sind; im Verhältnisse zum Gesamtgeschäfte sind das 8,4 v. H., 7,7 v. H. und 20,8 v. H. Beim ausländischen Geschäfte machtèn die Beiträge für das in Rückdeckung übernommene Geschäft 13,2 v. H. von den Beiträgen für das ganze ausländische Geschäft aus. Für Versicherungsleistungen wurden 5,552 Millionen Mark gewährt, nämlich 4,328 Millionen Mark für selbst abgeschlossene Versicherungen und 1,224 Millionen Mark für Rückversicherungen; von der Gesamtausgabe für ausländische Schäden kommen demnach 22,05 v. H. auf in Rückdeckung übernommene Versicherungen. Auf $1000 \mathscr{M}$ Beitragseinnahme kommen $772 \mathscr{N}$ Schadenzahlungen.

Außer den erwähnten großen Unternelımungen unterlagen der Reichsaufsicht noch einige Gegenseitigkeitsvereine und einige Eingetragene Genossenschaften m. b. H. von geringerer Bedeutung, die ihre Mitglieder gegen Haftpflicht oder auch gegen Unfall und Haftpflicht versichern. Eine Änderung gegenüber dem Vorjahre hat nicht stattgefunden, so daß hier wieder die Hauptergebnisse von 11 solchen Vereinen mitgeteilt werden können.

Die Zahl der Versicherungsscheine dieser 11 Unternehmungen belief sich Ende I 9 I 5 auf 68043 . Die Beiträge für das Geschäftsjahr betrugen für alle Unternehmungen zu-

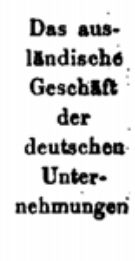 lundiseho Gesebart der Unter. mungen

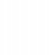


sammen $780206 \varkappa$. Davon wurden für Rückversicherungen $139669 \mathscr{M}$ wieder abgeführt. Verausgabt wurden für Schäden $442393 \mathscr{M}$ und für Verwaltungskosten $145994 \mathscr{N}$. Das Geschäftsjahr hat für 9 Vereine einen Gewinn von insgesamt $212533 \mathscr{N}$ (einschließlich $1582 \mathscr{M}$ Gewinnvortrag aus dem Vorjahre), für einen Verein einen Verlust von $1616 \mathscr{M}$ gebracht; ein Verein hat die Ausgaben gerade durch die Einnahmen gedeckt. Von dem Gewinne sind $148535 \mathscr{N}$ den gesetzlichen Rücklagen, $3000 \mathscr{M}$ den sonstigen Rücklagen und $460 \mathscr{M}$ den Garanten überwiesen worden; ferner sind $6100 \mathscr{N}$ an Tantiemen ausgegeben, $48703 \mathscr{H}$ den Versicherten gutgeschrieben, $5500 \mathscr{M}$ anderweit verwendet und $235 \mathscr{M}$ auf neue Rechnung vorgetragen.

Die Bilanz dieser Unternehmungen schließt mit $4216434 \mathscr{M}$ ab. Als Hauptposten der Vermögenswerte sind zu vermerken $3376496 \mathscr{M}$ Kapitalanlagen, $523953 \mathscr{M}$ Forderungen und $11593 \mu$ Kassenbestand; als Hauptposten der Verbindlichkeiten kommen in Betracht 630215 $\mathscr{N}$ Garantiekapital, $2016176 \mathscr{N}$ gesetzliche Rü̈cklagen, $198196 \mathscr{N}$ Prämienüberträge und $792014 \mathscr{N}$ Schadenrücklage.

Das deutsehe Geschsn aus. Indischer Gésellschaften

Zur Erläuterung des Umfanges des deutschen Geschäfts der ausländischen Unternehmungen in der Unfall- und Haftpflichtversicherung sollen die Tafeln II 10 bis 12 dienen. Es handelt sich wie früher nur um österreichische, ungarische und schweizerische Gesellschaften.

Die Basler Lebens-Versicherungs-Gesellschaft hat im Deutschen Reiche nur in der Unfallversicherung und in der Lebensversicherung Geschäftc betrieben, während die Foncière nur in der Unfallversicherung und in der Transportversicherung gearbeitet hat. Von den anderen Gesellschaften betrieben in Deutschland neben der Unfall- und Haftpflichtversicherung die Zürich auch die Einbruchdiebstahl- und die Kautionsversicherung, die Schweizerische Național-Versicherungs-Gesellschaft auch die Kinbruchdiebstahl-, die Glasversicherung und die Transportversicherung, die Schweizerische UnfallversicherungsAktiengesellschaft in Winterthur die Einbruchdiebstahlversicherung und die Kautionsund Bürgschaftsversicherung, die Providentia in Wien die Maschinenversicherung und die Erste Österreichische Allgemeine Unfall-Versicherungs-Gesellschaft in Wien die Wasserleitungsschäden- und Einbruchdiebstahlversicherung.

Acht ausländische Gesellschaften haben aus ihrem Unfallversicherungsgeschäft im Deutschen Reiche 1915 $5901141 \mathscr{M}$. Beiträge für selbst abgeschlossene Versicherungen und $5169 \mathscr{M}$ Beiträge für die in Rückdeckung ,übernommenen Versicherungen eingenommen, wovon $876597 \mathscr{M}$ für Rückversicherung weitergegeben wurden.

An dem gesamten Geschäfte der Gesellschaften in der Unfallversicherung war das deutsche Geschäft mit 13,3 v. H. der Beitragseinnahme und mit 22,6 v. H. der Ausgabe für Rückversicherungsbeiträge beteiligt.

In der Haftpflichtversicherung haben sechs ausländische Gesellschaften aus dem deutschen Geschäft $7057628 \mathscr{M}$ eingenommen, davon $113044 \mathscr{M}$ oder 1,6 v. H. für die in Rückdeckung übernommenen Versicherungen; diese Beitragseinnahme betrug 29,7 v. H. des gesamten Haftpflichtversicherungsgeschäfts. "Es wurden im deutschen Geschäfte $2021630 \mathscr{M}$ oder 28,6 v. H. der Beiträge, im Gesamtgeschäfte 8,5 v. H. der Beiträge für Rückversicherungen wieder ausgegeben.

Für selbst abgeschlossene Versicherungen hatten die Gesellschaften folgende Beträge bereitzustellen: 2722423 AH für Unfallversicherungsfälle, 2691988 , $M$ für Haftpflicht- 
versicherungsfälle, $247804 \mathscr{M}$ für laufende Renten und $106604 \mathscr{M}$ für Beitragsrückgewälırbeträge, zusammen $5768819 \mathscr{M}$, für Versicherungsfälle aus den in Rückdeckung übernommenen Versicherungen $15048 \mathscr{M}$ für Unfallversicherungsfälle, $247715 \mathscr{M}$ für Haftpflichtversicherungsfälle und $4449 \varkappa$ für sonstige Leistungen, zusammen $267212 \mathscr{M}$. Im Geschäftsjahr 1915 haben diese Gesellschaften insgesamt $6036031 \mathscr{A}$ für Schadenfälle, für Renten und für Prämienreserveergänzung verausgabt; in welchem Umfange hieran die Rückversicherer beteiligt sind, kann nicht angegeben werden. Diesen Schäden steht eine Rohbeitragseinnahme -- allerdings ohne Berücksichtigung der Überträge — von $12963938 \mathscr{M}$ gegenüber; von $1000 \mathscr{M}$ Beiträgen sind danach durchschnittlich $466 \mathscr{M}$ für Schäden ausgegeben.

Im Vergleiche des Berichtsjahrs mit dem Vorjahr ist in der Unfallversicherung die Zahl der Versicherungsscheine von 147112 auf 134606 oder um 8,5 v. H., die Beitragseinnahme von $8710864 \mathscr{M}$ auf $5906310 \mathscr{N}$ oder um 32,2 v. H. gefallen; bei der Haftpflichtversicherung ist die Zahl der Versicherungsscheine von 406881 auf 396455 oder um 2,6 v. H., die Beitragseinnahme von $9765370 \mathscr{M l}$ auf $7057628 \mathscr{H}$ oder um 27,7 v. H. gefallen. Das Geschäft ist also. auch hier, wie bei den deutschen Unternehmungen; infolge des Krieges zurückgegangen.

Zur Beurteilung des Vermögensstandes der ausländischen Gesellschaften sind in diese Statistik die Bilanzen der Gesellschaften aufgenommen, wie sie für das ganze Geschäft (nicht nur für das deutsche Geschäft) gelten. Dabei ist möglichst nach denselben Grundsätzen und in denselben Formen vorgegangen wie bei den deutschen Unternehmungen; es bedarf daher hier keiner besonderen Erklärungen.

Die Bilanz ist hier nur für sieben Gesellschaften zu geben ('Tafel II 14), da die der Basler Lebens-Versicherungs-Gesellschaft nach Gruppe I genommen ist.

Hinsichtlich der Kapitalanlagen dieser sieben Gesellschaften ist zu bemerken, daß die vier österreichischen Unternehmungen von ihrem ganzen Bestande von $103441573 \mathscr{M}$ in Grundbesitz $5171972 \mathscr{M}$ oder 5,0 v. H., in Hypotheken $14408884 \mathscr{M}$ oder 13,9 v. H., in Darlehen an öffentliche Körperschaften $2181240 \mathscr{M}$ oder 2,1 v. H., in Wertpapieren $74790293 \mathscr{M}$ oder 72,3 v. H., in Darlehen auf Wertpapiere $655098 \mathscr{M}$ oder 0,7 v. H., in Darlehen auf Versicherungen $5688717 \mathscr{N}$.oder 5,5 v. H. und in Wechseln 545369 $\mathscr{N}$ oder 0,5 v. H. angelegt hatten, und daß von dem gesamten Bestande (139831612 $\mathcal{M})$ der drei schweizerischen Gesellschaften $11948000 \mathscr{M}$ oder 8,5 v. H. in Grundbesitz, $53061413 \mathscr{M}$ oder 38,0 v. H. in Hypotheken, $74672633 \mathscr{M}$ oder 53,4 v. H. in Wertpapieren und $149566 \mathscr{H}$ oder 0,1 v. H. in Darlehen auf Versicherungen angelegt waren.

Ein eingehender Vergleich des ausländischen Geschäfts der deutschen Unternehmungen mit dem deutschen Geschäfte der ausländischen Unternehmungen auf dem Gebiete der Unfall- und Haftpflichtversicherung kann zwar nicht gegeben werden; indessen verdienen doch folgende Zahlen angeführt zu werden:

Im Jahre I 9 I 5 betrugen die Rohbeitragseinnahmen:

\begin{tabular}{|c|c|c|c|}
\hline & insgesamt & $\begin{array}{c}\text { für selbst } \\
\text { abgeschlossene Versicherungen }\end{array}$ & $\begin{array}{l}\text { für in Rückdeckung } \\
\text { übernommene Versicherungen }\end{array}$ \\
\hline$s \mathrm{~d}$ & $1000 \mu$ & $1000 \mu$ & $1000 \mathscr{H}$ \\
\hline $\begin{array}{l}\text { ausländischen Geschăfte } \ldots \ldots \ldots \ldots \\
\text { er ausländischen Gesellschaften aus } \\
\text { dem deutschen Geschäfte } \ldots \ldots \ldots \ldots\end{array}$ & $\begin{array}{r}7188 \\
\text { I } 2964\end{array}$ & $\begin{array}{r}6237 \\
12846\end{array}$ & II 8 \\
\hline
\end{tabular}

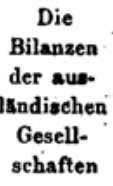

schaften

Das ius. lăndische Geschsift der deutschen und das deutseho Geschnet der auslandischen Gesellschaften 
Während also die Einnahmen an Rückversicherungsbeiträgen bei den deutschen Gesellschaften größer gewesen sind als bei den ausländischen Unternehmungen, bleiben die Beitragseinnahmen für das unmittelbare Geschäft bei den deutschen Gesellschaften hinter den entsprechenden Einnahmen der ausländischen Anstalten beträchtlich zurück; insgesamt haben die deutschen Gesellschaften für Unfall- und Haftpflichtversicherungen rund $5776000 \mathscr{M}$ weniger an Beiträgen aus dem Auslande bezogen, als die ausländischen Gesellschaften aus dem Deutschen Reiche eingenommen haben.

Für Versicherungsleistungen sind im Jahre I9 I 5 gezahlt worden:

\begin{tabular}{|c|c|c|c|}
\hline & $\begin{array}{l}\text { insgesamt } \\
1000 \text { M }\end{array}$ & $\begin{array}{c}\text { für selbst } \\
\text { abgeschlossene Versicherungen } \\
1000\end{array}$ & $\begin{array}{c}\text { für in Rĭckdeckung } \\
\text { übernommene Versicherungen } \\
1000\end{array}$ \\
\hline $\begin{array}{l}\text { on den deutschen Gesellschaften für } \\
\text { ausländische Versicherungen ..... }\end{array}$ & 5552 & (25) 10000 & 205000 \\
\hline $\begin{array}{l}\text { den auslāndischen Gesellschaften } \\
\text { für deutsche Versicherungen ..... }\end{array}$ & 6036 & 5769 & 267 \\
\hline
\end{tabular}

Näheres hierüber findet man in der "Entwickelung " S. 13.

\section{Gruppe III, Landwirtschaftliche Versicherung}

Für diese Gruppe kommen zur Zeit nur die Viehversicherung und die Hagelversicherung in Frage; sonstige landwirtschaftliche Versicherungen unterstanden im Jahre I 9 I 5 der Reichsaufsicht nicht.

\section{Viehversicherung}

Die Viehversicherung wird im Deutșchen Reiche außer von einigen größeren Unternehmungen von mehreren tausend kleinen Vereinen von lokaler Bedeutung betrieben. Davon unterliegt der Reichsaufsicht nur ein kleiner Bruchteil; dieser Bruchteil enthält wieder eine Anzahl Vereine von so untergeordneter Bedeutung, daß sich ihre eingehendere statistische Betrachtung nur in Verbindung mit. der Gesamtheit aller kleinen Unternehmungen lohnen würde, vgl. Veröffentlichungen des Kaiserlichen Aufsichtsamts igi6, S. 34 und 35.

- Die vorliegende Statistik beschränkt sich daher auf 1 Aktiengesellschaft, die 26 wichtigsten größeren Gegenseitigkeitsvereine sowie auf 7 sonstige Unternehmungen. Die in den früheren Statistiken unter den Gegenseitigkeitsvereinen aufgeführte Anhaltische ViehVersicherungs-Bank auf Gegenseitigkeit in Cöthen ist fortgelassen worden.

Die Aktiengesellschaft betätigt sich außer in der Viehlebens- und der Schlachtviehversicherung noch in mehreren sonstigen Viehversicherungsarten. DieSchlachtviehversicherung aufGegenseitigkeit des Vereins der Viehhändler zu Hannover beschränkt sich auf dieSchlachtviehversicherung. Die übrigen Gegenseitigkeitsvereine betreiben sämtlich die Viehlebensversicherung, 9 von ihnen gleichzeitig die Schlachtviehversicherung und 19 von ihnęn andere Arten der Viehversicherung. Daneben befaßt sich die Altenburger Vieh-Versicherungs-Gesellschaft auch mit der Sachschäden-Haftpflichtversicherung und der CentralViehversicherungs-Verein in Berlin mit der Verkehrsversicherung. Die sonstigen Unternehmungen (Einzelunternelımer) betätigen sich sämtlich und in erster Linie in der Schlachtviehversicherung; außerdem betreibt die Ostrauer Schlachtvieh-Versicherung auch die 
Viehlebensversicherung und die Schlesische Schlachtvieh-Versicherung auch die kurzfristige Nutzvieh- und Kuhkalbenversicherung.

Ausländische Unternehmungen waren in der Viehversicherung zum Geschäftsbetriel, im Deutschen Reiche nicht befugt.

Nach Tafel III 1 hatten die sämtlichen in Betracht genommenen 34 Unternehmungen im Berichtsjahre Versicherungen im Betrage von 848,039 Millionen Mark in Kraft; davon kommen 306,311 Millionen auf die Aktiengesellschaft, 368,584 Millionen (darunter 166,205 Millionen Mark gegen feste Beiträge) auf die 26 Gegenseitigkeitsvereine und 173,144 Millionen auf die 7 andern Unternehmer. Rückversicherungen finden sich nur bei der Aktiengesellschaft und bei den Gegenseitigkeitsvereinen. Von der Aktiengesellschaft waren 1,725 Millionen Mark in Rückdeckung übernommen und 44,393 Millionen Mark (gegen 40,741 Millionen Mark im Vorjahre) in Rückdeckung gegeben, von dem Gesamtbetrage der Versicherungen der Gegenseitigkeitsvereine waren 2,362 Millionen Mark in Rückdeckung übernommen und 11,027 Millionen Mark in Rückdeckung gegeben, so daß für eigene Gefahr die Aktiengesellschaft 261,918 Millionen Mark und die Gegenseitigkeitsvereine 357,557 Millionen Mark versichert hatten.

Der Gang der Versicherungssummen in den letzten Jahren ist in der beigegebenen Tafel bildlich dargestellt.

Die Reinbeitragseinnahme aller Unternehmungen, d.h. die im Laufe des Jahres von den Versicherten eingenommenen Beiträge nach Abzug der Rückversicherungsbeiträge, hat $11565496 \mathscr{M}$ (5579331 $\mathscr{M}$ Vorbeiträge, $734599 \mathscr{M}$ Nachschußbeiträge, $5927135 \mathscr{M}$ feste Beiträge und $675569 \mathscr{N}$ Rückversicherungsbeiträge, Tafel III 5) betragen.

Gegen feste Beiträge werden von den Gegenseitigkeitsvereinen in der Regel abgeschlossen: die Schlachtviehversicherung und die in Tafel III 2 unter sonstiger Viehversicherung zusammengefaßten Arten, nämlich die Rückversicherung, die Viehtransport-, die Manöver-, Weide-, Stuten-, Operations-, Ausstellungsversicherung usw.

Als Schäden für eigene Rechnung ohne Schadenermittelungskosten, aber unter Berücksichtigung des Zuwachses oder der Abnahme der Schadenrücklage und unter Einschluß des Erlöses für verwertetes Vieh sind $10826094 \mathscr{A l}$ zu verrechnen gewesen.

Eine Statistik über die Ursachen der Entschädigungspflicht im Jahre 1915 ist in dem Geschäftsberichte des Aufsichtsamts für 1915 gegeben, vgl. Veröffentlichungen 1916 S. $104 \mathrm{ff}$.

Die Ubersicht S. 50* und $51^{*}$ zeigt, wie sich die Beiträge und die Schäden auf die verschiedenen Versicherungsweisen, die Vieharten und deren Stückzahl und Wert verteilen. Sie beruht auf denselben Grundlagen wie Tafel III 2; es sind daher auch in dieser Ubersicht die Anteile der Rückversicherer überall eingeschlossen. Die Beiträge, von denen die Rabatte nicht abgesetzt sind, sind die Einnahmen im Geschäftsjahr; die Schäden sind die im Laufe des Geschäftsjahrs entstandenen Schäden. In dieser Zusammenstellung kommt beispielsweise besonders deutlich zum Ausdruck, wie sehr verschieden die Verhältnisse bei den verschiedenen Versicherungsarten liegen.

Seit dem Jahre 1905 hat der gesamte Versicherungsbestand nach Vieharten verteilt werden können; es mag deshalb hier eine kleine Ubersicht über die Stückzahl der versicherten Tiere und der zugehörigen Versicherungssummen für die letzten 5 Jahre Platz finden, vgl. S. 52*. In betreff der vorhergehenden Jahre sei auf die früheren Jahrgănge der Statistik und auf die "Entwickelung“ S. 29 verwiesen. 


\begin{tabular}{|c|c|c|c|c|c|c|}
\hline Vi e hart & Stürkzahl & $\begin{array}{c}\text { Ver- } \\
\text { sicherungs- } \\
\text { sunmen } \\
\mathscr{M}\end{array}$ & $\mathscr{M}$ & $\begin{array}{l}\text { Zahl der } \\
\text { Schäden }\end{array}$ & $\begin{array}{c}\text { Betrag der } \\
\text { Schäden } \\
\text { einschl. } \\
\text { Erlös } \\
\\
\mathscr{H}\end{array}$ & $\begin{array}{c}\text { Erlös } \\
\mu\end{array}$ \\
\hline I & 2 & 3 & 4 & 5 & 6 & 7 \\
\hline & & & & & Viehve & • \\
\hline $\begin{array}{l}\text { Pferde (Maultiere, Esel) } \ldots \ldots \\
\text { Rindvieh } \ldots \ldots \ldots \ldots \ldots \ldots \ldots \\
\text { Schafe und Ziegen } \ldots \ldots \ldots \ldots \\
\text { Schweine } \ldots \ldots \ldots \ldots \ldots \ldots \ldots \\
\text { Sonstiges Kleinvieh......... }\end{array}$ & $\begin{array}{r}252211 \\
918213 \\
37728 \\
1444457 \\
\quad .566 \\
\end{array}$ & $\begin{array}{r}242739562 \\
367495314 \\
184173^{2} \\
235897951 \\
6475^{\circ}\end{array}$ & $\begin{array}{r}7405387 \\
3739701 \\
27408 \\
1138514 \\
4351\end{array}$ & $\begin{array}{r}11430 \\
55312 \\
799 \\
34980 \\
30\end{array}$ & $\begin{array}{r}5920909 \\
4213070 \\
21437 \\
1503410 \\
2304\end{array}$ & $\begin{array}{r}468627 \\
1876706 \\
2823 \\
433524 \\
273\end{array}$ \\
\hline Zusammen .... & 2653175 & Vieh & $\begin{array}{c}12315361 \mid \\
\text { ebensvers }\end{array}$ & cherung & $\begin{array}{r}11261130 \\
\text { (1 Aktieng }\end{array}$ & $\begin{array}{r}2781953 \\
\text { sellschaft, }\end{array}$ \\
\hline $\begin{array}{l}\text { Pferde (Maultiere, Esel) } \ldots \ldots \\
\text { Rindvieh } \ldots \ldots \ldots \ldots \ldots \ldots \\
\text { Schafe und Ziegen } \ldots \ldots \ldots \ldots \\
\text { Schweine } \ldots \ldots \ldots \ldots \ldots \ldots \ldots \\
\text { Sonstiges Kleinvieh......... }\end{array}$ & $\begin{array}{r}179394 \\
46496 \\
688 \mathrm{I} \\
22455 \\
56 \mathrm{I}\end{array}$ & $\begin{array}{r}172451373 \\
19277241 \\
304078 \\
2424294 \\
64195\end{array}$ & $\begin{array}{r}5755219 \\
529019 \\
19606 \\
89023 \\
4334\end{array}$ & \begin{tabular}{rr|}
7716 \\
1765 \\
486 \\
1156 \\
129 \\
\end{tabular} & $\begin{array}{r}4961807 \\
624363 \\
17070 \\
72529 \\
2179\end{array}$ & $\begin{array}{r}428766 \\
210503 \\
1295 \\
3586 \\
273\end{array}$ \\
\hline Zusammen .... & 西 & 194521181 & 6397201 & Schla & chtviehve & sicherung \\
\hline $\begin{array}{l}\text { Pferde (Maultiere, Esel) } \ldots \ldots \\
\text { Rindvieh } \ldots \ldots \ldots \ldots \ldots \ldots \ldots \\
\text { Schafe und Ziegen } \ldots \ldots \ldots \ldots \\
\text { Schweine } \ldots \ldots \ldots \ldots \ldots \ldots \\
\text { Sonstiges Kleinvieh.......... }\end{array}$ & $\begin{array}{r}1 \\
778650 \\
22677 \\
1255441 \\
3 \\
\end{array}$ & $\begin{array}{r}100 \\
307538829 \\
1097186 \\
213816544 \\
55 \\
\end{array}$ & $\begin{array}{r}5 \\
2974047 \\
5454 \\
970327 \\
2\end{array}$ & \begin{tabular}{rr|}
- & \\
52 & 427 \\
31 & 266 \\
3 & 147 \\
- &
\end{tabular} & \begin{tabular}{r|}
- \\
3378441 \\
3078 \\
930626 \\
- \\
\end{tabular} & $\begin{array}{r}- \\
5^{82} 686 \\
1467 \\
409046 \\
- \\
\end{array}$ \\
\hline Zusammen .... & 2056772 & 522452714 & 3949835 & Sonstic & Viehve & sicherung \\
\hline $\begin{array}{l}\text { Pferde (Naultiere, Esel) } \ldots \ldots \\
\text { Rindvieh } \ldots \ldots \ldots \ldots \ldots \ldots \\
\text { Schafe und Ziegen } \ldots \ldots \ldots \ldots \\
\text { Schweine } \ldots \ldots \ldots \ldots \ldots \ldots \\
\text { Sonstiges Kleinvieh........ }\end{array}$ & $\begin{array}{r}72816 \\
93067 \\
8170 \\
16656 \mathrm{r} \\
2\end{array}$ & $\begin{array}{r}70288089 \\
40679244 \\
440468 \\
19657 \text { 1 } 13 \\
500\end{array}$ & $\begin{array}{r}1650163 \\
236635 \\
2348 \\
79164 \\
15\end{array}$ & \begin{tabular}{rr|}
3714 \\
$1 \quad 120$ \\
47 \\
2677 \\
1
\end{tabular} & $\begin{array}{r}959102 \\
210266 \\
1289 \\
100255 \\
125\end{array}$ & $\begin{array}{r}39861 \\
83517 \\
61 \\
20892 \\
-\quad\end{array}$ \\
\hline Zusammen .... & 340616 & $131065 \cdot 414$ & 1968325 & 7559 & 1271037 & 144331 \\
\hline
\end{tabular}

Anmerkung: Ein - bedeutet, daß die beobachteten Fälle weniger als $1 / 2 \%$ ausmachen. 


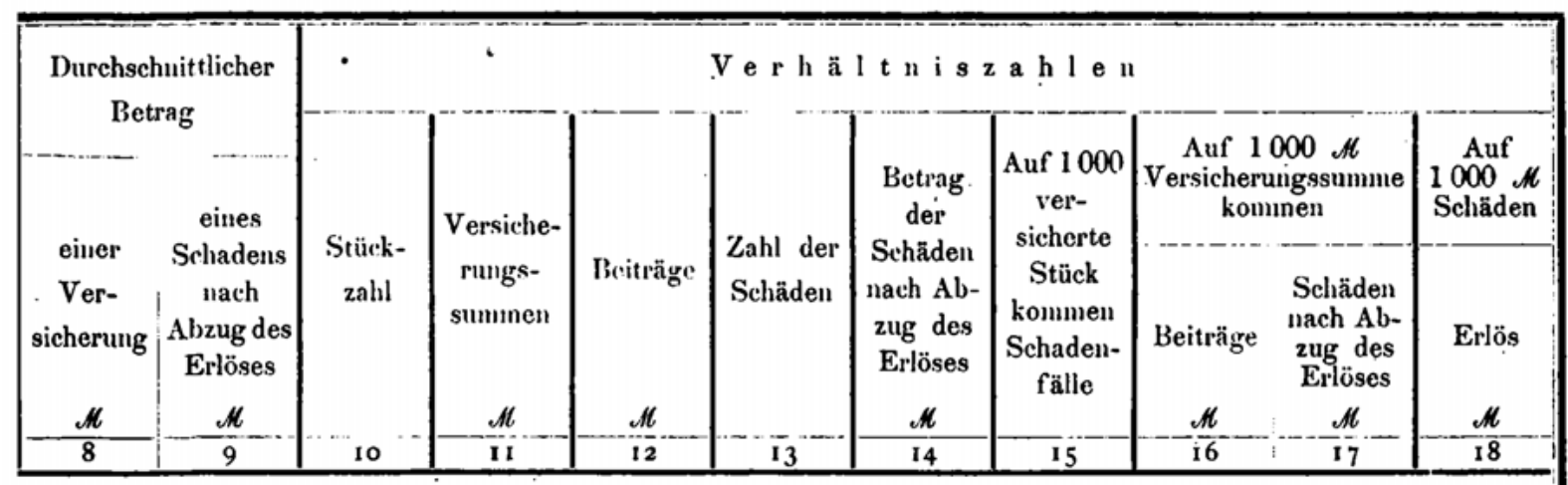

insgesamt

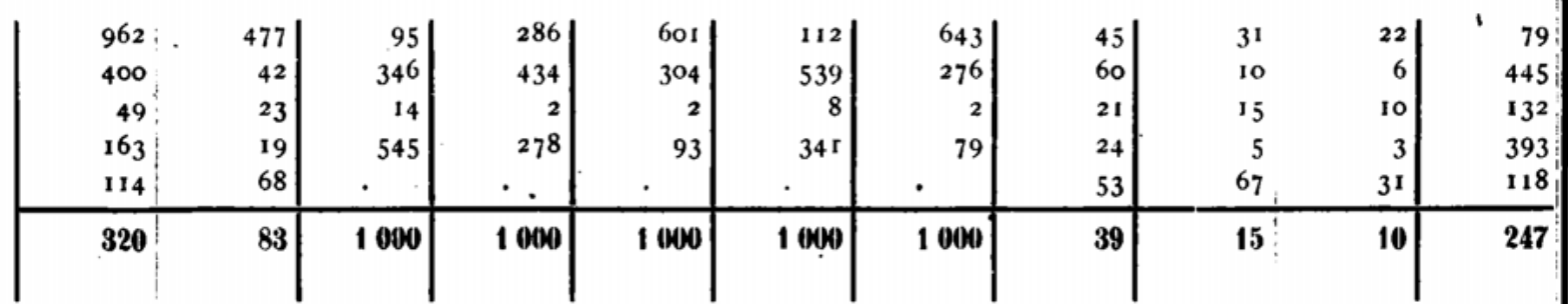

25 Gegenseitigkeitsvereine und 1 sonstige Unternelimung)

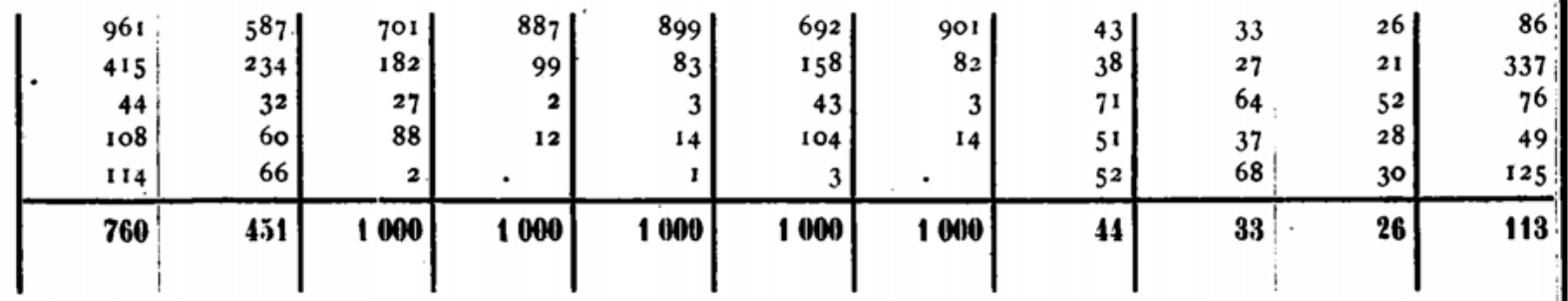

(17 Unternehmungen)

\begin{tabular}{|r|r|r|r|r|r|r|r|r|r|r|}
100 & - & & & - & 5 & - & - & 50 & - & - \\
395 & 34 & 379 & 589 & 753 & 625 & 774 & 67 & 10 & 6 & 468 \\
48 & 6 & 11 & 2 & 1 & 3 & 1 & 12 & 5 & 1 & 477 \\
170 & 17 & 610 & 409 & 246 & 372 & 225 & 25 & 5 & 2 & 440 \\
18 & - &. &. &. & - & - & - & - & - \\
\hline 254 & 28 & 1000 & 1000 & 1000 & 1000 & 1000 & 41 & 8 & 4 & 462 \\
\hline
\end{tabular}

(21 Unternehmungen)

\begin{tabular}{|r|r|r|r|r|r|r|r|r|r|r|}
965 & 248 & 214 & 536 & 839 & 492 & 816 & 51 & 23 & 13 & 42 \\
437 & 113 & 273 & 311 & 120 & 148 & 113 & 12 & 6 & 3 & 397 \\
54 & 26 & 24 & 3 & 1 & 6 & 1 & 6 & 5 & 3 & 47 \\
118 & 30 & 489 & 150 & 40 & 354 & 70 & 16 & 4 & 4 & 208 \\
250 & 125 &. &. &. &. &. & 500 & 30 & 250 & - \\
\hline 385 & 149 & 1000 & 1000 & 1000 & 1000 & 1000 & 22 & 15 & 9 & 114 \\
& & & & & & & & & &
\end{tabular}




\begin{tabular}{|c|c|c|c|c|c|c|c|c|}
\hline \multirow{2}{*}{ Geschäftsjahr } & \multicolumn{3}{|c|}{ Viehlebensversicherung } & \multicolumn{2}{|c|}{ Schlachtviehversicherung } & \multicolumn{3}{|c|}{ Sonstige Viehversicherung } \\
\hline & Pferde & Rindvieh & Schweine & Rindvieh & Schweine & Pferde & Rindvieh & Schweine \\
\hline & \multicolumn{8}{|c|}{ Zahl der versicherten Tiere } \\
\hline $1911 \ldots \ldots \ldots$ & 221089 & 90415 & 35368 & 815711 & 2286881 & 41985 & I 24799 & 415786 \\
\hline $1912 \ldots$. & 225035 & 81446 & 32118 & 762246 & 2105806 & 56834 & I1 8642 & 343890 \\
\hline $1913 \ldots$ & 223382 & $6660 \mathrm{I}$ & $27 \cdot 156$ & 706607 & I 9455 I 8 & $6494 \mathrm{I}$ & $1055^{89}$ & 265077 \\
\hline $1914 \ldots \ldots \ldots$ & 220691 & 54077 & 25860 & 700040 & I 895 I54 & 69775 & 97898 & 236606 \\
\hline \multirow[t]{2}{*}{$1915 \ldots \ldots \ldots$} & 179394 & 46496 & 22455 & 778650 & 1255441 & 72816 & 93067 & $16656 r$ \\
\hline & \multicolumn{8}{|c|}{ Versicherungssummen in $1000 \mathscr{M}$} \\
\hline $1911 \ldots$ & 181565 & 32713 & 3167 & 245671 & 257903 & 28857 & 49204 & $35 \circ 98$ \\
\hline $1912 \ldots$ & 191791 & 30888 & 2878 & 229339 & 248836 & 39521 & $48 \mathrm{I} 2 \mathrm{I}$ & 30836 \\
\hline $1913 \ldots$ & 193609 & 25818 & 2654 & 224544 & 232793 & 50698 & 48725 & 24883 \\
\hline $1914 \ldots$ & I 96692 & 22564 & 2630 & 232426 & 236563 & 52621 & 44060 & 22677 \\
\hline $1915 \ldots \ldots \ldots$ & $17245^{2}$ & 19277 & 2424 & 307539 & 213817 & 70288 & 40679 & 19657 \\
\hline
\end{tabular}

Zahlen sind bei den Versicherungssummen nicht streng vergleichbar, weil an einigen Stellen nicht der Betrag der überhaupt in Kraft gewesenen Versicherungen, sondern der Betrag der am Ende des Jahres laufenden Versicherungen eingestellt werden mußte. Bei der Aktiengesellschaft haben die Versicherungssummen um 1,619 Millionen Mark oder 0,5 v. H. und die Beiträge um 0,076 Millionen oder 3,2 v. H. zugenommen, die Schadenbeträge (ohne Abzug des Erlöses) um 0,296 Millionen oder 9,8 v. H. abgenommen. Bei den Gegenseitigkeitsvereinen haben die Versicherungssummen um 10,417 Millionen Mark oder 2,7 v. H., die Beiträge um 0,926 Millionen oder 10,3 v. H. und die Schadenbeträge (ohne Abzug des Erlöses) um 1,174 Millionen oder 14,3 v. H. abgenommen. Der Vergleich von 1914 mit I9I 3 hatte nach der vorjährigen Statistik hinsichtlich der Aktiengesellschaft bei den Versicherungssummen eine Abnahme um 0,927 Millionen Mark oder 0,3 v. H., bei den Beiträgen eine Abnahme um 0,110 Millionen oder 4,4 v. H. und bei den Schäden eine Abnahme um 0,578 Millionen oder 16,0 v. H. und hinsichtlich der Gegenseitigkeitsvereine bei den Versicherungssummen eine Abnahme um 5,167 Millionen Mark oder 1,3 v. H., bei den Beiträgen eine Abnahme um 0,749 Millionen oder 7,6 v. H. und bei den Schäden eine Abnahme um 0,869 Millionen oder 9,5 v.H. ergeben.

Bei den sonstigen Unternehmungen haben die Versicherungssummen um 46,280 Millionen oder 36,5 v. H. zugenommen, die Beiträge um 0,061 Millionen oder 5,7 v. $\mathrm{H}$. und die Schadenzahlungen um 0,354 Millionen oder 25,5 v. H. abgenommen.

Der Einfluß des Krieges hat sich also, wie zu erwarten war, I9I 5 stärker geltend gemacht als I9I4.

Die Gewinn- und Verlustrechnung ('Tafel III 4) stellt nur das Ergebnis des Berichtsjahrs dar; der Gewinnvortrag aus dem Vorjahr ist fortgelassen, auch sind die Prämienreserven und Schadenrücklagen nicht mit ihrem ganzen Betrag am Anfange des Jahres in Einnahme und am Schlusse des Jahres in Ausgabe nachgewiesen, sondern es sind nur die Änderungen, welche der Betrieb des Jahres zur Folge hatte, verrechnet (vgl. Tafel III 5).

Die gesamte Beitragseinnahme hat im Jahre $191512241065 \mathscr{N}$ betragen. Wie sich dieser Betrag auf Vorbeiträge, Nachschußbeiträge und feste Beiträge verteilt, ist 
bereits oben angegeben; es braucht hier nur noch hinzugefügt zu werden, daß die Nachschüsse 13,2 v. H. der Vorbeiträge aller Unternehmungen und 40,1 v. H. der Vorbeiträge derjenigen Unternehmungen ausgemacht haben, welche im Berichtsjahr überhaupt Nachschüsse erhoben haben. Verrechnet man auf die Beiträge die Prämienüberträge und die Rückversicherungsbeiträge, so erhält man die in die Betriebsrechnung eingestellte Beitragseinnahme. Die von einigen Vereinen durch die Gewinn- und Verlustrechnung geführten "Nachschußversicherungsbeiträge " oder "Nachschußrückversicherungsbeiträge " sind hier fortgelassen; es handelt sich nämlich um Beiträge für eine Nebenversicherung gegen etwaigen Nachschuß, die der Verein von seinen Mitgliedern einzieht, um sie zumeist vollständig an andere Versicherungsgesellschaften abzuführen, also um durchlaufende Posten, die den Geschäftsbetrieb nicht weiter berühren. Die Leistungen der Versicherer aus diesen Versicherungen sind bei den Nachschüssen eingestellt.

Die Nebenleistungen der Versicherten haben bei den Gegenseitigkeitsvereinen im Jahre I 9 I $5298762, \mu$, das sind 4,0 v. $\mathrm{H}$. der ganzen Beitragseinnahme oder 5,4 v. H. der Vorbeiträge, bei der Aktiengesellschaft $9675 \mathscr{M}$, das sind 0,3 v. H. der ganzen Beitragseinnahme, betragen. Dabei ist zu bemerken, daß bei den meisten Versicherungsgesellschaften als satzungsmäßige Leistung der Mitglieder zur gesetzlichen Rücklage bestimmte Hundertsätze des Vorbeitrags verrechnet und als Teile des Vorbeitrags nachgewiesen werden, also nicht bei den Nebenleistungen erscheinen.

Der Erlös aus verwertetem Vieh belief sich auf $2986081 \mathscr{M}$, d. h. 27,6 $\mathscr{M}$ von je $100 \mathscr{M}$ Schäden für eigene Rechnung. Hierbei ist zu beachten, daß zum Teil nicht nur der wirklich erzielte Erlös oder der bedingungsgemäß geśtattete Mindesterlös in Rechnung gestellt ist, sondern auch vielfach, insbesondere bei Versicherungen gegen Minderwert, der Unterschied zwischen Versicherungssumme und gezahltem Schadenbetrage angerechnet ist.

An Schadenermittelungskosten wurden $286551 \mathscr{N}$ oder $2,6 \mathscr{A}$ auf je $100 \mathscr{M}$ Schäden einschließlich Erlös gezahlt; an eigenen Verwaltungskosten und Steuern wurden $2643252 \mathscr{M}$ oder 22,5 v. H. der Beiträge für eigene Rechnung (Tafel III 5 Sp. 8) verausgabt.

Aus der Bilanz für Ende 1915, die nur für die Aktiengesellschaft und die Gegen- Die Bilanz seitigkeitsvereine gegeben wird, mögen folgende Posten angeführt werden:
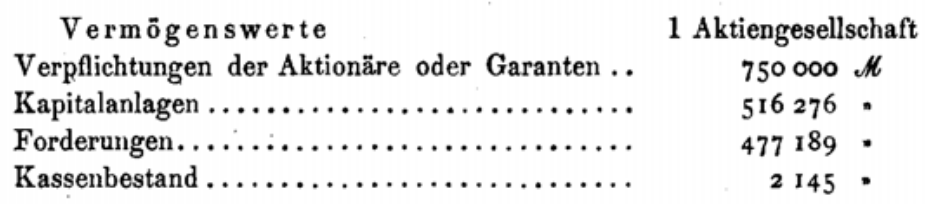

27 Gegenseitigkeitsvereine

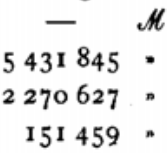

Verbindlichkeiten

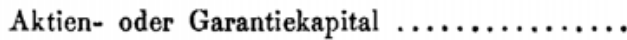

Gesetzliche Rücklage ..................

Sonderrücklagen.$\ldots \ldots \ldots \ldots \ldots \ldots \ldots \ldots \ldots$

Prämienüberträge......................

Schadenrücklage $\ldots \ldots \ldots \ldots \ldots \ldots \ldots \ldots \ldots \ldots$
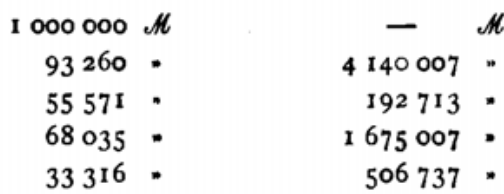

Die Kapitalanlagen der Aktiengesellschaft und der Gegenseitigkeitsvereine zusammen bestehen zu 79,4 v. H. in Wertpapieren; zu 4,3 v. H. in Grundbesitz, zu 16,2 v. H. in Hypotheken und zu 0,1 v. H. in Darlehen an öffentliche Körperschaften. Im Ver- 
gleiche mit dem Bestand am Ende des Vorjahrs waren im Besitze dieser Lnternehmung'n Ende I9I 5 rorhanden an Wertpapieren 831240 eth (dem Nennwert nach $918505 . /$ ) mehr, dagegen an Hypotheken $3001, H$ und an Grundbesitz $60, \mathscr{H}$ weniger. Die Forderungen hestehen bei der Gesamtheit zu 56,6 v. H. in Rückständen der Versicherten (einschl. der noch nicht gezahlten Nachschüsse): ins Gewicht fallen bei einigen (iesellschaften auch die Ausstände bei Agenten.

Die gesetzlichen Rücklagen haben gegen die Bilanz für Ende r 9 I 4 um 459764 ,/l oder um 12,2 v. H. zugenommen: die Sonderrücklagen vermehrten sich um 64514 , oder 35,1 v. $\mathrm{H}$.

Das aus.

Iandische Gesrhåft der Ausdehnung und des Čmfanges des darin enthaltenen ausländischen Geschäfts mögen folgende Nachrichten dienen.

Im Jahre I 9 I 5 haben wie in den Vorjahren nur 5 Versicherungsvereine auch im Auslande Geschäfte abgeschlossen, davon je einer in Belgien, Luxemburg und in der Schweiz und je zwei in Dänemark und Österreich. Diese Vereine hatten am Ende des Jahres im Ausland eine Summe von 3251201 th versichert. An Beiträgen haben sie im Berichtsjahr 15.5465 ett eingenommen, an Schäden sind 127959 eth gezahlt. Das ausländische Geschäft war also in der Viehversicherung nur unbedeutend; es betrug $3, \times$ vom Tausend der gesamten Versicherungssumme, 13,4 vom Tausend der Beitragseinnahme und $11, x$ rom Tausend der Schäden der Cnternehmungen.

\section{Hagelversicherung}

Von den im Deutschen Reiche im Jahre 1915 vorhanden gewesenen Hagelversicherungsunternehmungen unterstanden dem Auf'sichtsamte 4 Aktiengesellschaften und 14 Gregenseitigkeitsvereine. Davon sind in diese Statistik außer den Aktiengesellschaften 12 Vereine aufgenommen; die übrigen zwei sind von ganz lokalem Charakter und geringer Bedeutung, so daß von ilnnen nicht wohl alle Angaben, welche diese Statistik hringt, gefordert werden können.

Drei von den hier behandelten 12 Gegenseitigkeitsvereinen betreiben auch die Feuerversicherung, nämlich die (ireifswalder, die Mecklenburgische und die Schwedter IIagel- und Feuer-Versicherungs-Gesellschaft; eine, die Greifswalder, betreibt auch die Baulast- und die Einbruchliebstahlversicherung. Alle drei halten aber die Versicherungszweige in ihren Büchern getrennt und geben eine vollständige Gewinn- und Verlustrechnung und Bilanz für jeden Zweig. Diese vollständige Trennung ist auch hier beibehalten worden, so daß sich die hier für diese Gesellschaften gegebenen Zahlen lediglich auf die Hagelversicherung beziehen, wie die für dieselben Gesellschaften bei Gruppe IV gegebenen Zahlen lediglich das Feuerversicherungsgeschäft betreften.

Ausländische Hagelversicherungsgesellschaften haben, soweit bekannt. im Jahre 1915 im Deutschen Reiche nicht gearbeitet. 
Der Umfang des Gesehäfts im Berichtsjahre wird durch folgende Zahlen bezeichnet. Fs kamen insgesant 3 495,196 Millionen Mark auf 492966 selbst abgeschlossene Versicherungen oder im Durchschnitt $7090 \mathscr{M}$ auf einen Versicherungsschein.

Hierin sind 178,182 Millionen Mark enthalten, die zu 15922 Kollektiv- oder Gemeindeversicherungen gehören, welche in der vorstehenden Zahl der Versicherungen je nur als eine Versicherung gezählt sind, aber 163547 Teilnehmer hatten. Die durchschnittlich von einem Versicherten versicherte Summe stellt sich demnach bei der Gesamtheit auf $5456 \mathscr{M}$ und bei den Gemeinde- und Kollektivversicherungen allein auf $1089 \mathscr{M}$.

Von den Versicherungen kommen auf die Aktiengesellschaften 942,005 Millionen Mark und 135522 Versicherungsscheine oder $6951 \mathscr{M}$ im Durchschnitt für den Schein und auf die Gegenseitigkeitsvereine 2553,191 Millionen Mark und 357444 Versicherungen oder $7143 \mathrm{~d}$ im Durchschnitt für den Schein.

Die Rückversicherung hatte keine große Bedeutung. Es hatten in Rückdeckung übernommen vier Aktiengesellschaften zusammen 124,3i2 Millionen Mark und in Rückdeckung gegeben vier Gesellschaften 120,067 Millionen Mark, so daß die Aktiengesellschaften zusammen von 1066,347 Millionen Mark überhaupt übernommener Versicherungen 946,280 Millionen für eigene Rechnung behalten haben. Von den Gegenseitigkeitsvereinen haben zwei 0,163 Millionen Mark in Rückdeckung übernommen und 2,289 Millionen Mạk in Rückdeckung gegeben, so daß bei diesen Anstaiten 2 551,065 Millionen Mark für eigene Rechnung blieben.

Die Reinbeitragseinnahmen, d. h. die im Laufe des Jahres von den Versicherten Die Beitrage eingenommenen Beiträge, vermindert um die ausgegebenen Rückversicherungsbeiträge, haben insgesạmt betragen $29940638 \mathscr{M}$, wovon $11079026 \mathscr{M}$ auf die Aktiengesellschaften und $18861612 \mathscr{M}$ auf die Gegenseitigkeitsvereine entfielen (Tafel III 11).

Als Schäden für eigene Rechnung, ohne die Ermittelungskosten, aber unter Berücksichtigung der Änderung der Schadenrücklage im Laufe des Jahres (vgl. Tafeln III 11 und 13), sind insgesamt $18747773 \mathscr{M}$ zu verrechnen gewesen: $3181775 \mathscr{M}$ bei den Aktiengesellschaften und $15565998 \mathscr{M}$ bei den Gegenseitigkeitsvereinen. Als jedenfalls nicht zu kleine Zahl der Schäden (weil bei drei Gesellschaften nicht die Zahl der Schäden, sondern die Zahl der Anmeldungen angegeben ist) darf für die Gesamtheit 47666 angenommen werden, nämlich 12330 für die Aktiengesellschaften und 35336 für die Gegenseitigkeitsvereine. Es kommen also auf den Schadenfall im Durchschnitt mindestens: bei der Gesamtheit $393 \mathscr{M}$, bei den Aktiengesellschaften $258 \mathscr{M}$ und bei den Gegenscitigkeitsvereinen $441 \mathscr{M}$.

Als Entschädigungsabzüge sind insgesamt $1502909 \mathscr{M}$ einbehalten worden, $162706 \mathscr{M}$ von den Aktiengesellschaften und $1340203 \mathscr{M}$ von den Gegenseitigkeitsvereinen. Die Abzüge bestanden bei den Aktiengesellschaften ausschließlich in Anteilen der Geschädigten an den Frmittelungskosten; von den Abzügen der Gegenseitigkeitsvereine waren $552938 \mathscr{M}$ für Ermittelungskosten, $778019 \mathscr{M}$ für die gesetzlichen Rücklagen und $9246 \mathscr{N}$ für andere Zwecke bestimmt.

Die Prämienüberträge und die Schadenrücklage spielen bei der Hàgelversicherung natứgemäß nur eine ganz untergeordnete Rolle (vgl. Tafel III 13); man kann daher von diesen Rückstellungen absehen und folgende Zahlen als zutreffend annehmen: 
Von $1000 \mathscr{M}$ Beiträgen wurden für Schadenzahlungen nach Verrechnung der Entschädigungsabzüge verwendet:

\begin{tabular}{|c|c|c|c|c|c|}
\hline im Jahre.... & 1911 & $\begin{array}{l}1912 \\
804 \mu\end{array}$ & 1913 & 1914 & $\begin{array}{l}1915 \\
626.4\end{array}$ \\
\hline $\begin{array}{l}\text { on allen Unte } \\
\text { - den Aktie }\end{array}$ & $\begin{array}{l}573 m \\
271 .\end{array}$ & $\begin{array}{l}804 m \\
507 .\end{array}$ & $\begin{array}{l}579 \pi \\
258 .\end{array}$ & $\begin{array}{l}730 m \\
419 .\end{array}$ & $\begin{array}{l}020 m \\
287 m\end{array}$ \\
\hline - Gegenseitigkeitsvereinen...$\ldots \ldots \ldots \ldots \ldots$ & 767. & 927. & 746. & $850 \cdot$ & 825 . \\
\hline
\end{tabular}

Auf $1000 \mathscr{M}$ Versicherungssumme kamen:

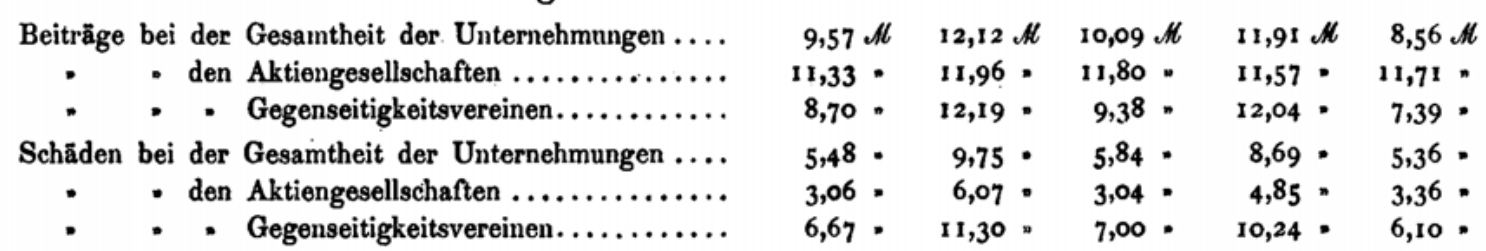

Vergleich Eine Vergleichung des Berichtsjahrs mit dem Vorjahre führt zu folgenden Erdes Berichts. gebnissen (Tafel III 11).

dem Vor.

jahre

Die Zahl der Versicherungen hat bei der Gesamtheit der Unternehmungen um 24894 oder 4,0 v. H. abgenommen; im besonderen hat sie bei den Aktiengesellschaften um 11994 oder 4,9 v. H. und bei den Gegenseitigkeitsvereinen um 12900 oder 3,5 v. H. abgenommen. Die Versicherungssummen sind bei der Gesamtheit der Unternehmungen um 372,657 Millionen Mark oder 11,5 v. H. gestiegen, und zwar bei den Aktiengesellschaften um 52,309 Millionen Mark oder 5,2 v. H. und bei den Gegenseitigkeitsvereinen um 320,348 Millionen Mark oder 14,3 v. H. Die Reinbeitragseinnahme (einschl. Nachschüsse) hat im Berichtsjahre gegenưber dem Vorjahre bei den Aktiengesellschaften um $760043 \mathscr{A}$ oder 7,4 v. H. zugenommen und bei den Gegenseitigkeitsvereinen um $7915642 \mathscr{A}$ oder 29,6 v. H. abgenommen. Die Schäden für eigene Rechnung sind bei den Aktiengesellschaften um $1146766 \mathscr{M}$ oder 26,5 v. H. und bei den Gegenseitigkeitsvereinen um $7192452 \mathscr{M}$ oder $31,6 \mathrm{v}$. H. niedriger gewesen als im Vorjahre.

Die in Tafel III 12 gegebene Gewinn- und Verlustrechnung stellt nur das Ergebnis des Berichtsjahrs dar; der Gewinnvortrag aus dem Vorjahre ist fortgelassen; auch sind die sonstigen Uberträge nicht mit ihrem vollen Betrage zu Anfang des Jahres in Einnahme und am Schlusse des Jahres in Ausgabe nachgewiesen, sondern es sind nur die Änderungen, welche der Betrieb des Geschäftsjahrs zur Folge hatte, verrechnet (vgl. 'Tafel III 13).

Die Beiträge haben bei den Aktiengesellschaften $12407703 \mathscr{M}$ betragen, wovon für Rückversicherung $1328677 \mathscr{M}$ wieder ausgegeben wurden. Die Gegenseitigkeitsvereine haben $18374993 \mathscr{N}$ Vorbeitrag und $527267 \mathscr{M}$ Nachschußbeitrag erhoben; für Rückversicherung haben sie $40648 \mathscr{H}$ wieder ausgegeben; der Nachschußbeitrag machte 2,9 v. H. von dem Vorbeitrag aus, oder wenn man die Greifswalder, die Schleswig-Holsteiner in Kiel, die Neubrandenburger, den Holsteinischen H.V.V. in Neustadt und die Oldenburger H.V.G. ausschließt, weil sie die Beiträge durch Umlage nach Bedarf aufbringen, 34,3 v. H. Gewisse von mehreren Gegenseitigkeitsvereinen unter der Bezeichnung *Rückversicherungsbeiträge « durch die Gewinn- und Verlustrechnung geführte Posten sind hier fortgelassen; es handelt sich nämlich um Beiträge für eine Nebenversicherung gegen etwaigen Nachschuß, die der Verein von seinen Mitgliedern einzieht, um sie meist vollständig an andere Versicherungsgesellschaften abzuführen, also um durch- 
laufende Posten, die das Geschäftsergebnis nicht weiter berühren. Die Leistungen der Versicherer aus diesen Versicherungen sind bei den Nachschüssen eingestellt.

An Nebenleistungen hatten die Versicherten zu zahlen bei den Aktiengesellschaften $90111 \mathscr{M}$, das sind 0,7 v. H. der Beiträge, und bei den Gegenseitigkeitsvereinen $2432688 \mathscr{M}$, das sind 12,0 v. H. der gesamten Beiträge oder 13,2 v. H. der Vorbeiträge. Die Nebenleistungen bestehen bei den Gegenseitigkeitsvereinen zum größten Teile $(1979767 \mathscr{M})$ in satzungsmäßigen Beiträgen zur gesetzlichen Rücklage, die entweder nach der Höhe des Vorbeitrags oder der Entschädigung bemessen werden; setzt man diese ab, so bleiben $452921 \mathscr{M}$, das sind 2,4 v. H. der gesamten Beiträge oder 2,5 v. H. der Vorbeiträge. Die von einigen Gegenseitigkeitsvereinen erhobenen Legegelder (Sicherheitsleistungen) sind hier außer acht gelassen.

An Schadenermittelungskosten sind ohne die oben erwähnten Abzüge bei allen Unternehmungen $1034555 \mathscr{M}$ oder 5,5 $\mathscr{M}$ auf je $100 \mathscr{M}$ Schadenzahlung, bei den Aktiengesellschaften $367699 \mathscr{M}$ oder 11,6 $\mathscr{H}$ und bei den Gegenseitigkeitsvereinen $666856 \mathscr{M}$ oder $4,3 \mathscr{M}$ auf je $100 \mathscr{M}$ Schäden entstanden.

Das Geschäft des Jahres 1915 hat sämtlichen Aktiengesellschaften einen Gewinn von zusammen $5245235 \mathscr{M}$ gebracht. Das gilt vom gesamten Geschäfte. Scheidet man bei den einzelnen Gesellschaften das Ergebnis der Vermögensverwaltung aus, so ermäßigt sich der Gewinr jener Gesellschaften auf $4743255 \mathscr{N}$. Die Gegenseitigkeitsvereine decken ihren Verlust meist schon vor Abschluß der Rechnung aus der gesetzlichen Rücklage oder durch Nachschüsse (vgl. nEntwickelung " S. 50 u. 51); darüber hinaus haben fünf Vereine für das Berichtsjahr einen Gewinn von zusammen $329328 \mathscr{M}$ nachgewiesen.

Aus der Bilanz für ı 9 I 5 , die für die Aktiengésellschaften zusammen mit $45345255 \mu$ und für die Gegenseitigkeitsvereine zusammen mit $15695910 \mathscr{N}$ abschließt, mögen folgende Posten angeführt werden:

\begin{tabular}{|c|c|c|c|}
\hline Vermögenswerte & 4 Aktiengesellschaften & 12 Gegenseitigkei & itsvereine \\
\hline Verpflichtungen der Aktionäre oder Garanten & $19620900 \mathscr{N}$ & - & $\mathscr{M}$ \\
\hline Kapitalanlagen.$\ldots \ldots \ldots \ldots \ldots \ldots \ldots \ldots \ldots$ & 21715472 & 11145336 & - \\
\hline Forderungen $\ldots \ldots \ldots \ldots \ldots \ldots \ldots \ldots \ldots \ldots$ & 3926820 & 4416.496 & n \\
\hline Kassenbestand $\ldots \ldots \ldots \ldots \ldots \ldots \ldots \ldots \ldots$ & 82063 & 129709 & • \\
\hline Verbindlichkeiten & & & \\
\hline Aktien- oder Garantiekapital............. & $25528500 \mathscr{A H}$ & 一 & $\mathscr{H}$ \\
\hline Gesetzliche Rücklage.$\ldots \ldots \ldots \ldots \ldots \ldots$ & 1877772 . & 10271532 & $\cdot$ \\
\hline Sonderrücklagen $\ldots \ldots \ldots \ldots \ldots \ldots \ldots \ldots$ & 11655030 . & I 523430 & - \\
\hline Schadenrücklage $\ldots \ldots \ldots \ldots \ldots \ldots \ldots \ldots$ & 52891 . & 715061 & - \\
\hline
\end{tabular}

Uber die Kapitalanlagen gibt Tafel III 15 folgende Aufschlüsse. Von $1000 \mathscr{M}$ Kapitalanlagen kamen Ende I9 I5 auf:

\begin{tabular}{|c|c|c|c|c|c|c|c|}
\hline & $\begin{array}{c}\text { Grund- } \\
\text { besitz }\end{array}$ & $\begin{array}{l}\text { Hypo- } \\
\text { theken }\end{array}$ & $\begin{array}{c}\text { Darlehen } \\
\text { an öffentliche } \\
\text { Körperschaften }\end{array}$ & $\begin{array}{c}\text { Darlehen } \\
\text { auf } \\
\text { Wertpapiere }\end{array}$ & $\begin{array}{l}\text { Wert- } \\
\text { papiere }\end{array}$ & Wechsel & $\begin{array}{l}\text { Sonstige } \\
\text { Anlagen }\end{array}$ \\
\hline $\begin{array}{l}\text { bei den Aktiengesellschaften } \ldots \ldots \ldots \ldots \\
\text { bei den Gegenseitigkeitsvereinen } \ldots \ldots \ldots\end{array}$ & $\begin{array}{l}23 \mathscr{N} \\
43 \cdots\end{array}$ & $\begin{array}{l}77 \mathscr{K} \\
140 \cdot\end{array}$ & $-\mu$ & $\begin{array}{c}9 \mu \\
0,3\end{array}$ & $\begin{array}{l}861 \mathscr{N} \\
755 *\end{array}$ & $\begin{array}{l}30 \% \\
57=\end{array}$ & $-\mu$ \\
\hline
\end{tabular}

Im Vergleiche mit der Bilanz des Vorjahrs haben abgenommen die Darlehen auf Wertpapiere um $1000 \mathscr{M}$ und der Hypothekenbestand um $36537 \mathscr{M}$, dagegen zugenommen der Grundbesitz um $136843 \mathscr{M}$ und der Buchwert der Wertpapiere um $4919332 \mathscr{M}$. 
Dan aus-

landische Geschaft

Uber die Bedeutung des ausländischen Geschäfts der deutschen Hagelversicherungsunternehmungen, das in den vorstehenden Zahlen ïberall eingeschlossen ist, sind folgende Angabẹn zu machen:

Im ganzen haben vier Aktiengesellschaften und drei Gegenseitigkeitsvereine auch im Auslande Geschäfte betrieben. In Ungarn und in Luxemburg war je eine Gesellschaft tätig, in Rußland arbeiteten zwei, in den Niederlanden drei und in Österreich vier Anstalten. Die Versicherungssumme belief sich auf 14,704 Millionen Mark oder 4,1 vom Tausend der von allen Unternehmungen überhaupt versicherten Summe; die durchschnittliche Höhe einer selbst abgeschlossenen ausländischen Versicherung stellte sich auf $10858 \mathscr{M}$. Die Rohbeitragseinnahme aus dem ausländischen Geschäfte betrug $225528 \mathscr{M}$ oder 7,2 vom Tausend der gesamten Rohbeiträge; die Schäden betrugen $136057 \mathscr{M}$ oder 7,3 vom Tausend der gesamten Schäden.

\section{Gruppe IV, Feulerversicherung (einschl. Versicherung mittelbarer Schäden) sowie die Versicherungen gegen Sturmschäden, Wasserleitungsschäden und Einbruchdiebstahl}

Die Versicherung mittelharer Schsden

Seitdem mit dem Inkrafttreten des Versicherungsvertragsgesetzes die Schranken gefallen sind, welche bis dahin in dem größten Teile von Deutschland der Versicherung des durch den Eintritt eines Sachschadens entgehenden Gewinns, soweit hierbei die Feuerversicherung in Betracht kam, gezogen waren, haben die meisten der bei uns arbeitenden größeren Feuerversicherungsgesellschaften die Versicherung solcher mittelbarer Schäden in den Formen der Mietverlust-, der Betriebsverlust- und der Zuckerpreisdifferenzversicherung in ihren Geschäftsplan aufgenommen. Der Umfang dieser Geschäfte ist naturgemäß noch nicht beträchtlich, immerhin wird es von Wert sein, die Entwickelung von Anfang an statistisch verfolgen zu können; deshalb sind schon vom Jahre igi i ab einige Zusammenstellungen darüber mitgeteilt. Wegen des unmittelbaren Zusammenhangs dieser Versicherungen mit der Feuer- oder der Wasserleitungsschädenversicherung sind sie mit diesen Zweigen hier bei Gruppe IV behandelt. Es wird jedoch einstweilen nicht nötig sein, in diesem Texte näher auf sie einzugehen; der Hinweis auf die Tafeln wird genügen.

Von den deutschen Unternehmungen, die Versicherungen der Gruppe IV abschließen, unterstanden im Jahre I915 der Reichsaufsicht 45 Aktiengesellschaften und 35 Gegenseitigkeitsvereine. Die zahlreichen Feuerversicherungsvereine auf Gegenseitigkeit, welche wegen der Beschränkung ihres Geschäftsbetriebs auf einen Bundesstaat der Aufsicht der Landesbehörden unterstehen oder welche gemäß $§ 3$.Abs. 2 des Versicherungsaufsichtsgesetzes der Landesaufsicht überwiesen worden sind und denen auch in ihrer Gesamtheit keine allzu große Bedeutung zukommt, können in dieser Statistik nicht behandelt werden, weil sie nicht an das Aufsichtsamt zu berichten brauchen.

Von den in der vorjährigen Statistik bei Gruppe IV bearbeiteten 46 Aktiengesellschaften hat die Freia, Bremen-Berliner Versicherungs-Aktiengesellschaft in Berlin ihren Versicherungsbestand auf den Globus, Versicherungs-Aktien-Gesellschaft, Hamburg, durch Fusion übertragen. 
Die Ilanseatische Versicherungs-Aktien-(iesellschaft von 1877 in Hamburg hat ihren Geschäftsbetrieb auf dic Betriebsverlustversicherung ausgedehnt, während die Union, Allgemeine Versicherụngs-Aktien-Gesellschaft zu Berlin, die Zuckerpreisdifferenzversicherung, und die Oldenburger Versicherungs-Gesellschaft, die Wasserleitungsschädenversicherung aufinahmen.

Der Nordstern, Feuer-Versicherungs-Aktien-Gesellschaft in Berlin-Schöneberg, die Victoria, Feuer-Versicherungs-Aktien-Gesellschaft in Berlin und die Preußische NationalVersicherungs-(resellschaft zu Stettin haben die Zuckerpreisdifferenzversicherung fallen lassen.

Von den 45 Gesellschaften betrieben 40 die Feuerversicherung neben andern Zweigen, die übrigen 5 befaßten sich nicht mit der Feuerversicherung, sondern soweit die Versicherungszweige der Gruppe IV in Betracht kommen - nur mit einem oder mehreren, der Nebenzweige, von denen die Einbruchdiebstahlversicherung der wichtigste ist.

Von den $3 \overline{5}$ unter Reichsaufsicht stehenden Feuerversicherungsvereinen auf Gegenseitigkeit sind in der vorliegenden Statistik 24 ausführlich behandelt; zu den in der vorjäbrigen Statistik behandelten Vereinen ist die im Berichtsjahre zugelassene MobiliarBrand- und Einbruchdiebstahl-Versicherungskasse des Zentralverbandes der Kommunal(Gemeinde-) Beamten Preußens a. G. in Barmen, und die Brand- und Einbruchsschadenkasse Deutscher Lokomotivführer (Reichsverband), Versicherungsverein a. G. in Berlin, hinzugekommen. Von diesen 24 Vereinen beschränken sich nur 6 auf die Feuerversicherung, 3 weitere betreiben daneben die Hagelversicherung; die übrigen arbeiten außer in der Feuerversicherung noch in dem einen oder andern der Nebenzweige der Sachversicherung. Die drei Vereine, die auch Hagelversicherungen abschließen, führen aber - wie schon bei Gruppe III, S. 54* erwähnt - die verschiedenen Versicherungszweige vollständig voneinander getrennt, so daß hier nur auf das Feuer- und Einbruchdiebstahlversicherungsgeschäft eingegangen $\mathrm{zu}$ werden braucht.

Hinsichtlich der ausländischen Versicherungsunternehmungen, die sich mit den zu Gruppe IV gehörigen Versicherungszweigen beschäftigen, ist zu bemerken, daß, wie im vorigen Jahre die englischen, die französische und eine belgische fortgelassen worden sind. Der Hauptteil der Bestände dieser Gesellschaften ist durch Haftungs- und Ubergangsverträge bei deutschen Gesellschaften sichergestellt worden. Das Nähere hierüber findet sich in der Versicherungsstatistik für Igrq (S. 60*), es crübrigt sich, hier darauf zurückzukommen.

Wir beschränken demnach unsern Bericht für I9I5 auf 15 ausländische Feuerversicherungsgesellschaften. Davon arbeiteten, soweit Versicherungszweige der Gruppe IV in Frage kommen, im Deutschen Reiche 2 nur in der Feuerversicherung; weitere 8 betrieben die Feuerversicherung neben andern Zweigen und die übrigen 5 befaßten sich nicht mit der Feuerversicherung selbst, sondern nur mit den Nebenzweigen.

Bei den folgenden Ausführungen sowie bei der Betrachtung der Täfeln ist, soweit es sich. um Feuerversicherung handelt, im Auge zu behalten, daßstets die Gesamtheit der Versicherungen dargestellt ist; eine Trennung nach Risiken konnte nirgends vorgenommen werden; ebensowenig hat die Hausratfeuerversicherung von der Liegenschaftsversicherung geschieden werden können. 
Uber den Umfang des Geschäfts der deutschen Unternehmungen in der Feuerversicherung Ende 1914 und Ende 1915 gibt Tafel IV 1 Auskunft. Danach liefen an selbst abgeschlossenen Versicherungen einschließlich der in Rückdeckung gegebenen Teile:

- Ende I914

Ende 191.5

bei den Aktiengesellschaften ....... I1 179592 Vers. über 123650 Mill. $\mathscr{M} 11378491$ Vers. über 128910 Mill. $\mathscr{A}$

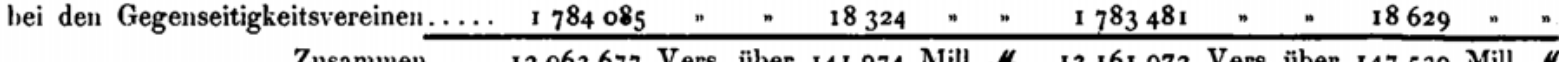
Zusammen .... 12963677 Vers. über 141 974 Mill. $\mathscr{H}$ 13 161 972 Vers. über 147539 Mill. $\mathscr{M}$

Derim unmittelbaren Geschäfte durchschnittlich auf eineVersicherung entfallendeBetrag hat sich, wie die folgende Zusammenstellung zeigt, in der letzten Zeit nur wenig geändert.

Durchschnittlich auf eine selbst abgeschlossene Versicherung entfallender Betrag in Mark Ende des Jahres

\begin{tabular}{|c|c|c|c|c|c|}
\hline Aktiengesellschaften, Gesantgeschäft ....... & $\begin{array}{c}1911 \\
10964\end{array}$ & $\begin{array}{c}1912 \\
11069\end{array}$ & $\begin{array}{c}1913 \\
10964\end{array}$ & $\begin{array}{c}1914 \\
11060\end{array}$ & $\begin{array}{l}1915 \\
11329\end{array}$ \\
\hline ktiengesellschaften, inländisches Geschäft .... & I I 034 & 11137 & II 114 & 11195 & I 534 \\
\hline ktiengesellschaften, ausländisches Geșchäft ... & 10130 & 10290 & 9447 & $95^{83}$ & 9046 \\
\hline regenseitigkeitsvereine $\ldots \ldots \ldots \ldots \ldots \ldots$ & 9954 & 10119 & IO I 22 & 10279 & 10445 \\
\hline & IO 820 & I0 934 & IO 845 & I0 953 & 11209 \\
\hline
\end{tabular}

Die Aktiengesellschaften hatten außerdem noch Versicherungen in Höhe von 19639 Millionen Mark am Ende 1914 und in Höhe von 15074 Millionen Mark am Ende 1915 in Rückdeckung genommen, so daß die gesamte Versicherungssumme Ende 19I4 143289 und Ende 1915 143984 Millionen Mark betrug. In dieser Summe sind alle diejenigen Beträge doppelt enthalten, welche von einer Gesellschaft, die in dieser Statistik behandelt wird, versichert und bei einer ebensolchen Gesellschaft in Rückdeckung gegeben sind. Dasselbe gilt von den unten angeführten Zahlen über die Gesamtversicherungssumme bei den andern Versicherungszweigen. Der Anteil des mittelbaren Geschäfts am Gesamtgeschäfte beträgt 10,5 v. H. Von derı Gegenseitigkeitsvereinen haben zwei den geringen Betrag von zusammen 69 Millionen Mark in Rückdeckung übernommen.

Rechnet man zur Feuerversicherung die Versicherung mittelbarer Schäden (Mietverlust-, Betriebsverlust- und Zuckerpreisdifferenzversicherung) hinzu, so belief sich die gesamte Versicherungssumme Ende 1915 bei den Aktiengesellschaften auf 144215 und bei den Gegenseitigkeitsvereinen auf 18705 Millionen Mark. Der davon für eigene Rechnung behaltene Teil stellte sich:

\begin{tabular}{|c|c|c|}
\hline bei den Aktiengesellsch & $\begin{array}{c}\text { Ende 1914 } \\
84240 \text { Mill. } \mathscr{M}\end{array}$ & $\begin{array}{l}\text { Ende } 1915 \\
82880 \text { Mill. } \mu\end{array}$ \\
\hline den Gegenseitigkeitsvereinen auf.. & $15623 \cdots \cdots$ & $15757 \times$ \\
\hline
\end{tabular}

und betrug in Tausendsteln der überhaupt versicherten Summen:

$\begin{array}{lll}\text { bei den Aktiengesellschaften ....... } & 610 & 595 \\ \text { bei den Gegenseitigkeitsvereinen .. } & \mathbf{8 4 3} & \mathbf{8 4 2}\end{array}$

Die Tafel IV 2 behandelt lediglich das deutsche Feuerversicherungsgeschäft der deutschen Aktiengesellschaften; aus dieser Tafel in Verbindung mit Tafel IV 1 ergibt sich folgende Teilung des Geschäfts:

Selbst abgeschlossene Versicherungen Ende 1914 Ende 1915 inländisches Geschäft ............. 10244043 Vers. über 114685 Mill. $\mathscr{A}$ 10 440937 Vers. über 120429 Mill. $\Perp$ ausländisches Geschäft........... 935549 " . 8966 " * 937554 • " 8481 • •

Von dem mittelbaren Geschäfte der Aktiengesellschaften kamen Ende I9I4 auf das inländische Geschäft 6522 Millionen Mark und auf das ausländische Geschäft 13117 Millionen Mark, Ende 1915 auf das inländische Geschäft 6319 Millionen Mark und auf das ausländische Geschäft 18755 Millioṇen Mark. 
Das gesamte inländische Feuerversicherungsgeschäft der Aktiengesellschaften erstreckte sich Ende 1914 über 121206 Millionen Mark und Ende 1915 über 126748 Millionen Mark; das gesamte ausländische Geschäft bezifferte sich Ende 1914 auf 22083 Millionen Mark und Ende I 9 I 5 auf 17 236. Millionen Mark. Der Anteil der mittelbaren Versicherungen an der Gesamtheit betrug danach beim inländischen Geschäft I9 I 4 5,4 v. H. und I9 I 5 4,9 v. H., beim ausländischen dagegen 19 I 4 59,4 v. H. und I9 5 $50,8 \mathrm{v} . \mathrm{H}$.

Von den Gegenseitigkeitsvereinen haben nur drei (der Versicherungsverein Deutscher Eisenbahnbediensteten, die Feuer- und Einbruchdiebstahl-Versicherungsanstalt des Verbandes Deutscher Beamtenvereine und die Gothaer Feuerversicherungsbank) ausländische Geschäfte, und zwar in geringem Maße, abgeschlossen. Alle zusammen hatten Ende I9I4 im Ausland 2066 Versicherungen über 46 Millionen Mark und Ende I9 I5 1999 Versicherungen über 45 Millionen Mark in Kraft.

Uber den Umfang der Mietverlust-, der Betriebsverlust-und der Zuckerpreisdifferenzversicherung geben die Tafeln IV 3 bis 7 Auskunft.

In der Sturmschädenversicherung haben im Jahre I9I5 dieselben beiden Aktiengesellschaften gearbeitet wie in den Vorjahren; der Erfolg ist auch in diesem Jahre kein großer gewesen. Nach Tafel IV 8 liefen nämlich Ende I9I4 insgesamt 2461 Versicherungen über 227,9 Millionen Mark und Ende I9 I 52525 Versicherungen mit 224,5 Millionen Mark, so daß die Zahl der Versicherungen um 64 zugenommen und die versicherten Summen ${ }^{\circ}$ um 3,3 Millionen Mark abgenommen haben. Im Auslande wurde dieser Zweig nicht betrieben. Für eigene Rechnung wurde nur der geringe Betrag von 40 Millionen Mark behalten. Rückversicherungen wurden von diesen beiden Gesellschaften nicht übernommen.

In der Wasserleitungsschädenversicherung haben Ende 1915 27 deutsche Aktiengesellschaften gearbeitet. Neu hinzugekommen ist die Oldenburger Versicherungs-Gesellschaft.

Nach Tafel IV 9 liefen an selbst abgeschlossenen Versicherungen Ende 1914 138119 Versicherungen über 6169 Millionen Mark und Ende 1915 136630 Versicherungen über. 6176 Millionen Mark, was einen Abgang von 1489 Versicherungen und einen Zugang von 7 Millionen Mark Versicherungssumme bedeutet.

Die Gesellschaften hatten außerdem noch Versicherungen in Höhe von 133,5 Millionen Mark zu Ende 1914 und in Höhe von 213,0 Millionen Mark zu Ende 1915 in Rückdeckung genommen, so daß die gesamte Versicherungssumme Ende I9I5 6389 Millionen Mark betrug. Von den Versicherungssummen kamen Ende 1914 und Ende I9I5 96,0 v. H. auf das deutsche Geschäft. Der weitaus größte Teil der Versicherungen, nämlich 5564 Millionen, wurde für eigene Rechnung behalten.

Von den Gegenseitigkeitsvereinen ist im Berichtsjahre diese Versicherungsart nur von der Concordia, Hannoversche Feuer-Versicherungs-Gesellschaft, betrieben worden, und zwar nur unmittelbar und nur innerhalb des Deutschen Reichs. Das gesamte Geschäft umfaßte am Ende des Berichtsjahrs 69 Versicherungen über $4092000 \mathscr{M}$.

Sehr viel bedeutender ist das Geschäft in der Einbruchdiebstahlversicherung. Es haben in diesem Zweige Ende I9 I 543 Aktiengesellschaften gearbeitet. Die.Freia, Bremen-Berliner Feuer-Versicherungs-Aktiengesellschaft in Berlin hat ihren Versicherungsbestand auf den Globus, Versicherungs-Aktien-Gesellschaft Hamburg durch Fusion übertragen. 
Von den selbst abgeschlossenen Versicherungen der deutschen Aktiengesellschaften kamen:

Ende $19 i_{4}$

Ende 1915

auf das inläıdische Geschäft...... I 132 I67 Vers. über 14720 Mill. $\mathscr{I} \quad$ I 114136 Vers. über 14526 Mill. $\mathscr{M}$

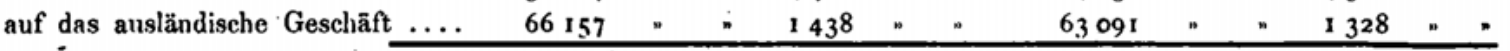

Zusaınmen .... I 198324 Vers. über 16 158 Mill. $\mathscr{A} \quad 1177227$ Vers. über 15854 Mill. $\mathscr{N}$

Das unmittelbare inlänđische Geschäft hat demnach um 18031 Versicherungen und 193 Millionen Mark, das ausländische um 3066 Versicherungen und 110 Millionen Mark abgenommen. In Rückdeckung übernommen hatten diese Gesellschaften außerdem im inländischen Betrieb am Ende des Vorjahrs 2476 und am Ende des Berichtsjahrs 2664 Millionen Mark, so daß die gesamte Versicherungssumme des deutschen Geschäfts Ende I 9 I 417195 Millionen Mark und Ende I9 517190 Millionen Mark betrug. Im ausländischen Geschäfte waren in Rückdeckung genommen Ende I 9 I 4 2112 und Ende 19i5 1 j41 Millionen. Mark; demnach ist die gesamte Versicherungssumme des ausländischen Geschäfts von 3551 auf 1869 ) Millionen Mark zurückgegangen. Der Anteil des mittelbaren Geschäfts am Gesamtgeschäft in der Einbruchdiebstahlversicherung belief sich danach beim inländischen Geschäft Ende I914 auf 14,4 und Ende 1915 auf 15,5 v. H., beim ausländischen Geschäft auf 59,5 bezw. 53,7 v. H.

Von den Gegenseitigkeitsvereinen haben in der Einbruchdiebstalılversicherung im Berichtsjahre 13 gearbeitet, zwei mehr als im Vorjahre, nämlich die Mobiliar-, Brand- und Einbruchdiebstahl-Versicherungskasse des Zentralverbandes der Kommunal(Gemeinde-) Beamten Preußens a. G. in Barmen und die Brand- und Einbruchschadenkasse Deutșcher Lokomotivführer (Reichsverband) Versicherungsverein a. G. in Berlin. Das Geschäft war auch im Jahre 1915 noch gering, es stellte sich Ende des Jahres auf insgesamt 84182 Versicherungsscheine über 842 Millionen Mark. Es handelt sich lediglich um unmittelbare deutsche Versicherungen.

Alle 56 Unternehmungen zusammen hatten Ende 1915 Versicherungen in Höhe von 20900 Millionen Mark in Kraft, davon liefen 14543 Millionen für eigene Rechnung.

Der

Umfang des

deutschen Gesohsins der aus.

Indischen Gesellschaften

Bei den ausländischen Versicherungsunternehmungen sehen wir, wie bereits oben erwähnt, von den englischen Gesellschaften, sowie von der französischen Gesellschaft und von der Belgischen Allgemeinen Feuer-Versicherungs-Aktien-Gesellschaft in Brüssel ab und berichten nur über Io Gesellschaften, wobei wir uns wie bisher auf deren deutsches Geschäft beschränken.

Diese Gesellschaften hatten im Deutschen Reiche selbst abgeschlossene Feuerversicherungen Ende 1914 in Höhe von 5542 Millionen Mark und Ende 19 I $_{5}$ in Höhe von 5620 Millionen Mark; in Rückdeckung waren zu denselbeñ Zeitpunkten Versicherungen im Betrage von 261 und von 307 Millionen Mark übernommen; der gesamte Versicherungsbestand betrug also in der Feuerversicherung am Schlusse des Vorjahrs 5803 und am Schlusse des Geschäftsjahrs 5927 Millionen Mark. Im Jahre 19 5 haben die selbst abgeschlossenen Versicherungen um 7.8 Millionen und die in Rückdeckung übernommenen Versicherungen um 46 Millionen Mark zugenommen.

Der Anteil des mittelbaren Geschäfts am Gesamtgeschäft ist von 4,5 v. H. auf 5,2 v. H. gestiegen.

Uber das geringfügige deutsche Geschäft dieser Gesellschaften in der Versicherung mittelbarer Schäden unterrichten die Tafeln IV 14 und 15. 
Von den ausländischen Gesellschaften, die im Deutschen Reiche in der Wasserleitungsschädenversicherung gearbeitet haben, kommen hier nur drei in Betracht. Neu hinzugekommen ist die Nye Danske Brandforsikrings-Selskab in Kopenhagen. Es wurden nur unmittelbare Versicherungen abgeschlossen. Das Geschüft umfaßte am Ende des Berichtsjahrs 586 Versicherungen über 1ə̆ Millionen Mark, gegenüber 521 Versicherungen über 13 Millionen Mark am Ende des Vorjahrs.

In der Einbruchdiebstahlversicherung berichten wir über zwölf ausländische Gesellschaften; von diesen haben neun lediglich unmittelbare, drei auch mittelbare Geschäfte betrieben. Nach Tafel IV 17 waren an selbst abgeschlossenen Versicherungen Fnde 1914 761 Millionen Mark und Ende 1915 741 Millionen Mark in Kraft. Rechnet man die in Rückdeckung übernommenen Versicherungen hinzu, so erhält.man für den Bestand des deutschen Geschäfts am Schlusse des Vorjahrs 858 und für den Bestand am Schlusse des Berichtsjahrs 842 Millionen Mark.

Der Wert aller im Deutschen Reiche gegen Feuerschaden versicherten Gegenstände, wie er sich aus der Summe aller im Reiche unmittelbar versicherten Beträge ergibt, kann aus den vorstehenden Angaben allein nicht entnommen werden. Abgesehen von den verhältnismäßig kleinen Summen, die bei den kleinen Gegenseitigkeitsvereinen und bei den unter Landesaufsicht stehenden privaten Unternehmungen versichert sind, fehlen noch die Versicherungen von 48 öffentlichen Feuerversicherungs-Sozietäten. Uber den Umfang dieser Versicherungen veröffentlicht der Verband der öffentlichen Feuerversicherungs-Anstalten jährlich in den "Mitteilungen für die öffentlichen Feuerversicherungs-Anstalten " Zusammenstellungen, deren Zuverlässigkeit nicht zu bezweifeln ist. Mit Hilfe dieser Angaben kann man den gesamten Feuerversicherungsbestand im Deutschen Reiche annähernd folgendermaßen feststellen:

Es waren an deutschen Werten versichert in Millionen Mark:

\begin{tabular}{|c|c|c|c|c|c|c|}
\hline 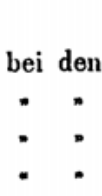 & $\begin{array}{l}\text { deutschen } \\
\text { ausländisch } \\
\text { öffentlichen }\end{array}$ & $\begin{array}{l}\text { Aktiengesellschaften } \ldots \ldots \ldots \ldots \ldots \ldots \\
\text { Gegenseitigkeitsvereinen } \ldots \ldots \ldots \ldots \\
\text { hen Gesellschaften insgesaint. } \ldots \ldots \ldots \\
\text { Feuerversicherungs-Austalten } \ldots \ldots\end{array}$ & $\begin{array}{l}\text { Eude } 1914 \\
114685 \\
18278 \\
9006 \\
89530 \\
\end{array}$ & $\begin{array}{r}\text { v. H. } \\
49,5 \\
7,9 \\
3,9 \\
38,7 \\
\end{array}$ & $\begin{array}{c}\text { Ende } 1915 \\
120429 \\
18585 \\
843 \\
90896 \\
\end{array}$ & $\begin{array}{r}\text { v.H. } \\
52,2 \\
8,0 \\
0 ; 4 \\
39,4 \\
\end{array}$ \\
\hline & & Zusammen . & 231499 & 100,0 & 230753 & 100,0 \\
\hline
\end{tabular}

Die Rohbeitragseinnahme im Berichtsjahr und die im Berichtsjahr entstandenen Schäden aller Arteǹ von Risiken, jedoch ohne. Schadenermittelungskosten, sind für die deutschen privaten Unternehmungen in den Tafeln IV 18 bis 24 und für das deutsche Geschäft der ausländischen Gesellschaften in den Tafeln IV 25 bis 29 zusammengestellt.

In der Feuerversicherung haben danach im Jahre I9 540 deutsche Aktiengesellschaften $255663106 \mathscr{M}$ und 24 Gegenseitigkeitsvereine $44816620 \mathscr{M}$, alle diese Unternehmungen zusammen $300479726 \mathscr{H}$ an Beiträgen eingenommen; in diesem Jahre sind Schäden (einschließlich des Anteils der Rückversicherer, aber ohne Schadenermittelungskosten) bei den Aktiengesellschaften im Betrage von 146222849 $\mathcal{M}$, bei den Gegenseitigkeitsvereinen im Betrage von $10.723103 \mathscr{M}$, bei allen Anstalten zusammen im Betrage von $156945952 \mathscr{M}$ entstanden. Dabei ist zu beachten, daß einige Gegenseitigkeitsvereine, um Nachschüsse zu vermeiden, Vorbeiträge erheben, die den 
regelmäßigen Bedarf weit übersteigen und den daraus entstehenden Überschuß den Versicherten wieder zuführen (vgl. Tafel IV 40).

Der durchschnittliche Betrag eines Schadens aus selbst abgeschlossenen Feuerversicherungen (ohne Ermittelungskosten) stellte sich bei den Aktiengesellschaften im inländischen Geschäft auf $586 \mathscr{M}$, im ausländischen Geschäft auf $816 \mathscr{M}$ gegen $442 \mathscr{N}$ bezw. $1011 \mathscr{M}$ im Vorjahre.

Bei den Gegenseitigkeitsvereinen hat der Schadenfall aus selbst abgeschlossenen Versicherungen im inländischen Geschäfte durchschnittlich $498 \mathcal{H}$, im ausländischen Geschäfte (23 Fälle) durchschnittlich $595 \mathscr{M}$ erfordert.

Uber die Versicherung mittelbarer Schäden führen die Tafeln 19 bis $21 \mathrm{zu}$ folgenden Frgebnissen:

\begin{tabular}{|c|c|c|c|c|c|c|}
\hline \multirow[t]{3}{*}{ en ragennssen. } & \multicolumn{2}{|c|}{ Aktiengesellschaften } & \multicolumn{2}{|c|}{$\begin{array}{c}\text { Gegenseitigkeits- } \\
\text { vereine }\end{array}$} & \multicolumn{2}{|c|}{ Zusanmen } \\
\hline & Beiträge & Schäden & Beiträge & Schäden & Beiträge & Schäden \\
\hline & $\mathscr{H}^{\circ}$ & $\mathscr{H}$ & $\mathscr{N}^{\circ}$ & $\mathscr{H}$ & $\mathscr{M}$ & $\mathscr{N}$ \\
\hline Nietverlustversicherung $\ldots \ldots \ldots \ldots$ & 140613 & 16057 & 7685 & 77 & 148298 & 16134 \\
\hline Betriebsverlustversicherung $\ldots \ldots \ldots$ & 507693 & 39818 & 32746 & 22 & 540439 & 39840 \\
\hline Zuckerpreisdifferenzversicherung ..... & 8,824 & - & - & - & 8824 & - \\
\hline
\end{tabular}

Die beiden Gesellschaften, die in der Versicherung gegen Sturmschäden gearbeitet haben, erzielten (Tafel IV 22) aus diesem Geschäft eine Beitragseinnahme von $162887 \mathscr{N}$, welche nur aus dem deutschen Betriebe herrührte. An Schäden sind aus selbst abgeschlossenen Versicherungen 245 mit $31519 \mathscr{M}$ (ohne Ermittelungskosten) zu verzeichnen gewesen; der durchschnittliche Betrag eines Schadens stellte sich demnach auf $129 \mathscr{N}$.

Die siebenundzwanzig Aktiengesellschaften, die in derVersich erung gegen Wass erleitungsschäden gearbeitet haben, erzielten aus diesem Geschäfte (Tafel IV 23) eine Beitragseinnahme von $3175520 \mathscr{M}$. An Schäden sind $1197512 \mathscr{M}$ (ohne Ermittelungskosten) zu verzeichnen gewesen. Der durchschnittliche Betrag eines Schadens aus selbst abgeschlossenen Versicherungen stellte sich beim inländischen Geschäft auf $53 \mathscr{N}$, beim ausländischen Geschäft auf $52 \mathscr{N}$.

In der Einbruchdiebstahlversicherung ('Tafel IV 24) haben 43 deutsche Aktiengesellschaften $15476958 \mathscr{M}$ an Beiträgen eingenommen und $3727487 \mathscr{M}$ für Schäden verausgabt; die 13 andern Unternehmungen haben $646320 \mathscr{M}$ Beiträge eingezogen und $99724 \mathscr{N}$ für Schäden gezahlt.

Der durchschnittliche Betrag eines Schadens aus den selbst abgeschlossenen Versicherungen stellte sich bei den Aktiengesellschaften (ohne Ermittelungskosten) im inländischen Geschäft auf $188 \mathscr{M}$ und im ausländischen Geschäft auf $547 \mathscr{M}$, bei den Gegenseitigkeitsvereinen auf $133 \mathscr{M}$.

b) der aus. lăndischen Gesellschaften

Für das deutsche Feuerversicherungsgeschäft der berücksichtigten 10 ausländischen Gesellschaften gelten folgende Zahlen (Tafel IV 25). Die Beitragseinnahme belief sich auf $10594936 \mathscr{M}$; für Schäden waren $5825423 \mathscr{M}$ aufzuwenden. Von den Beiträgen stammten 94,2 v. H. aus selbst abgeschlossenen Versicherungen und 5,8 v. H. aus Rückversicherungen, von den Schäden betrafen 94,0 v. H. selbst abgeschlossene Versicherungen und 6,0 v. H. in Rückdeckung übernommene Versicherungen.

Für die Versicherung mittelbarer Schäden haben die ausländischen Gesellschaften in Deutschland insgesamt $10775 \mathscr{N}$ an Beiträgen eingenommen und $32640 \mathscr{N}$ für Schäden ausbezahlt. 
Für das deutsche Wasserleitungsschädenversicherungsgeschäft der drei ausländischen Gesellschaften hat die Beitragseinnahme $11513 \mathscr{M}$ betragen; für Schäden sind $4197 \mathscr{N}$ gezahlt worden.

Das deutsche Einbruchdiebstahlversicherungsgeschäft der hier in Betracht kommenden 12 ausländischen Gesellschaften, das im wesentlichen unmittelbar betrieben wird, zeigt sich auch hinsichtlich der Beitragseinnahme und der Schadenleistungen ('Tafel IV 29) als wenig bedeutend. Die Beitragseinnahme hat nur $924544 \bumpeq$ betragen, für Schäden sind $271408 \varkappa$ zu zahlen gewesen.

Wie oben die Versicherungssummen annähernd für das gesamte unmittelbare deutsche Feuerversicherungsgeschäft ermittelt wurden, so können mit Hilfe der vorstehenden Zahlen und der Angaben in den "Mitteilungen für die öffentlichen FeuerversicherungsAnstalten " auch die Schadenbeträge, die den deutschen Geschädigten zugeflossen sind, angenähert festgestellt werden.

\begin{tabular}{|c|c|c|c|c|}
\hline & & chadenz & ingen & \\
\hline & in Jahrc & $9 ! 4$ & im Jahre & 1915 \\
\hline & $1000 \mathscr{A l}$ & v. H. & $1000 \mathscr{H}$ & v. H. \\
\hline deutsche Aktiengesellschaften $\ldots \ldots \ldots \ldots \ldots \ldots \ldots$ & 89963 & 50,1 & 93410 & 58,9 \\
\hline $\quad$ Gegenseitigkeitsvereine................. & 15102 & 8,4 & I0 533 & 6,6 \\
\hline $\begin{array}{l}\text { ausländische Gesellschaften mit über } 100000 \mathscr{A} \text { Bei- } \\
\text { tragseimmahme aus dem deutschen Geschäft ........ }\end{array}$ & 5555 & 3,1 & 5477 & 3,4 \\
\hline $\begin{array}{l}\text { ausländische Gesellschaften mit unter } 100000 \mathscr{H} \text { Bei- } \\
\text { tragseimnahme aus dem deutschen Geschäft......... }\end{array}$ & 70 & $0, \mathbf{r}$ & 89 & $\mathbf{0}, \mathbf{r}$ \\
\hline deutsche öffentliche Feuerṿersichcrungs-Anstalten ..... & 68767 & 38,3 & 49115 & 31,0 \\
\hline Zusammen .... & 179457 & 100,0 & 158624 & 100,0 \\
\hline
\end{tabular}

Auch in diesen Zahlen kommt der Ubergang der meisten deutschen Versicherungen bei englischen Gesellschaften auf deutsche Aktiengesellschaften deutlich zum Ausdruck.

Weit mehr tritt der Einfluß des Krieges zutage, wenn wir das ausländische (reschäft der deutschen Unternehmungen betrachten. Durch die Verkehrserschwerungen infolge des Krieges und die gleich nach Kriegsausbruch von den feindlichen Staaten erlassenen Zahlungsverbote an feindliche Ausländer wurde das deutsche Auslandsgeschäft hart getroffen. Die Beitragseinnahme ist, wie die folgende Zusammenstellung zeigt, um 13 Millionen Mark kleiner gewesen als im Vorjahre. Die Zahlungen für ausländische Schäden sind um 19 Millionen Mark kleiner gewesen als I914.

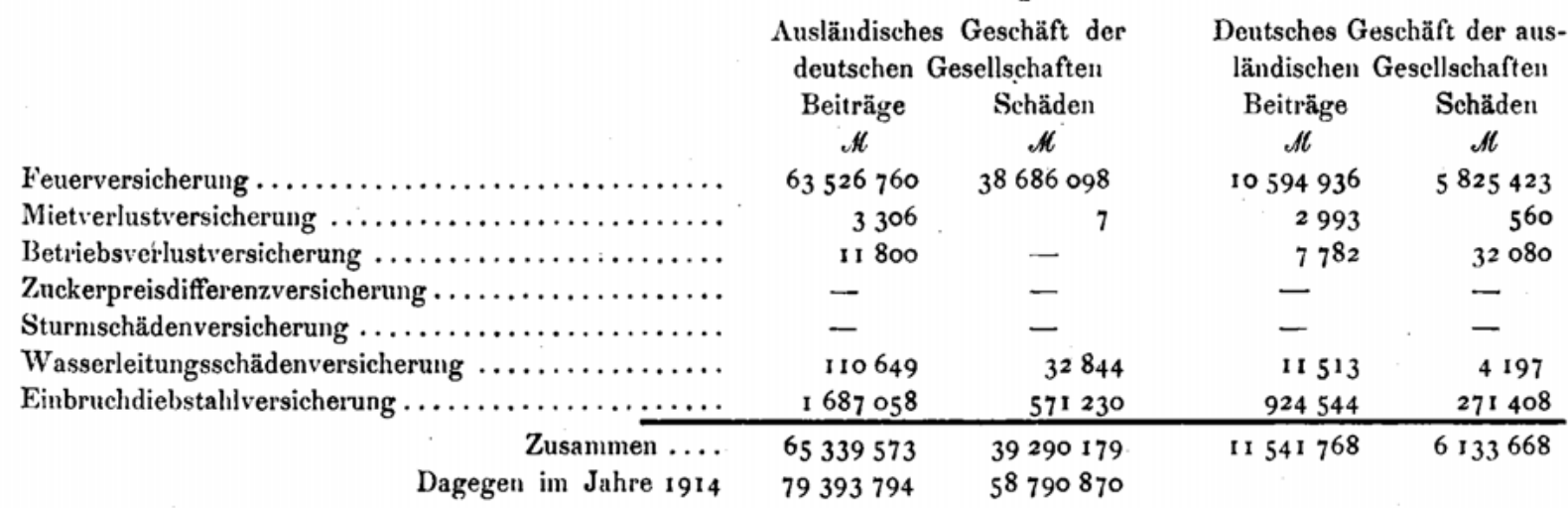

Im Jahre I 9 I 5 haben, um nur diejenigen Länder zu nennen, in denen deutsche Gesellschaften besonders zahlreich vertreten sind, in der Feuerversicherung gearbeitet in den Niederlanden 26, in der Schweiz 22, in Belgien 21, in Dänemark 14, in Ǒsterreich 
und in Schweden je 13, in Luxemburg 11, in China und in Siam je 10, in Ägypten und in Mexiko je 9, in der Türkei, in Norwegen, in Guatemala und auf den Philippinen je 7, in Deutsch-Südwestafrika, in Niederländisch-Indien, in Chile und in Kolumbien je 6 usw. -- Die Nebenzweige der Feuerversicherung haben naturgemäß erst weniger weite Verbreitung gefunden; doch arbeiteten in der Einbruchdiebstahlversicherung im Jahre 1915 in der Schweiz bereits 20, in den Niederlanden 16, in Belgien und in Österreich je 9, in Dänemark 6, in Luxemburg und in Schweden je 5 usw.; Wasserleitungsschädenversicherung haben in Dänemark und in der Schweiz je 7, in Österreich 5, in den Niedderlanden 3, in Schweden 2, in Belgien und in Ungarn je eine deutsche Gesellschaft betrieben.

Die Feuerversicherungs-Aktiengesellschaften arbeiten außer auf dem Gebiete der Feuerversicherung meistens auch noch in anderen Versicherungszweigen, ohne daß sie in ihren Büchern die sämtlichen Einnahme- und Ausgabeposten streng nach Geschäftszweigen trennten. Es ist jedoch seit einer Reihe von Jahren angängig, die Verwaltungskosten vollständig auf die einzelnen Zweige aufzuteilen, so daß die Betriebs-Einnahmen den Betriebs-Ausgaben nebst Verwaltungskosten und Steuern gegenübergestellt und somit der aus dem Versicherungsgeschäft allein herrührende Betriebs-Gewinn des Berichtsjahrs hergeleitet werden kann (Tafel IV 30 bis 33). Der Gewinn aus dem Gesamtgeschäfte wird in der Bilanz ('Tafel IV 38) nachgewiesen.

Der Gleichförmigkeit wegen sind die Aktiengesellschaften und die Gegenseitigkeitsvereine, die nur einen Geschäftszweig pflegen, ebenso behandelt worden.

Bei Gruppe IV sind als Betriebs-Einnahmen die Beiträge des Geschäftsjahrs für eigene Rechnung (Jahreseinnalıme nach Abzug der Ristorni und der verausgabten Rückversicherungsbeiträge), die Nebenleistungen der Versicherten, die Ersparnis aus der Schadenrücklage des Vorjahrs und die Abnahme der Prämienüberträge zusammengefaßt; als Betriebs-Ausgaben kommen die Zahlungen und Rückstellungen für Schäden, die im Geschäftsjahr entstanden sind (nach Abzug der Leistungen der Rückversicherer, aber einschließlich der Schadenermittelungskosten), die Leistungen für gemeinnützige Zwecke, der Zuschuß zur Schadenrücklage des Vorjahrs und die Zunahme der Prämienüberträge in Betracht.

Bei den deutschen Unternehmungen betrugen in der Feuerversicherung einschließlich Mietverlust-, Betriebsverlust- und Zuckerpreisdifferenzversicherung:

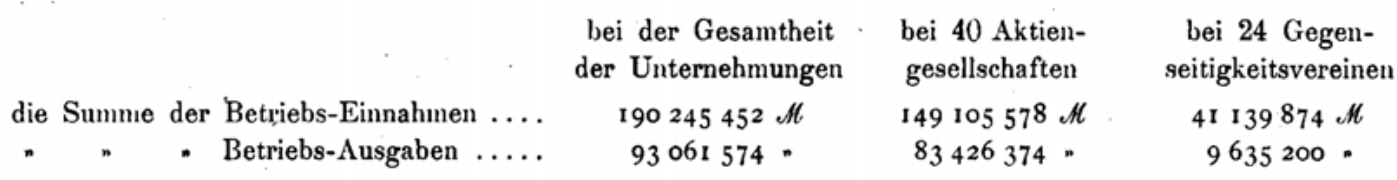

Von je $1000 \mathscr{M}$ dieser Betriebs-Einnahmen kamen:

\begin{tabular}{|c|c|c|c|}
\hline & $\begin{array}{l}\text { bei der Gesamtheit } \\
\text { der Unternehmungen. }\end{array}$ & $\begin{array}{l}\text { bei den } 40 \text { Aktien- } \\
\text { gesellschaften }\end{array}$ & $\begin{array}{l}\text { bei den } 24 \text { Gegen- } \\
\text { seitigkeitsvereinen }\end{array}$ \\
\hline auf Reinbeiträge.................. & $927 \mathscr{H}$ & $913 \mathscr{H}$ & $977 \mathscr{M}$ \\
\hline - Nebenleistungen der Versicherten ... & $9 \cdots$ & 8 & $13=$ \\
\hline$\because$ Ersparnis aus der Schademrücklage ... & $\because 59$ " & $73 \cdot$ & $7 \cdots$ \\
\hline - Abnahme der Prämienüberträge..... & $5 n$ & $6 \cdot$ & $3 n$ \\
\hline & $1000 \mathscr{H}_{-}$ & I $000 \mathscr{M}$ & $1000 \mathscr{A}$ \\
\hline
\end{tabular}


Von je 1000 et der Betriebs-Ausgaben kamen:

\begin{tabular}{|c|c|c|c|}
\hline & $\begin{array}{l}\text { bei der Gesanitheit } \\
\text { der Unternehmunge॥ }\end{array}$ & $\begin{array}{l}\text { bei den } 40 \text { Aktien- } \\
\text { gesellschaften }\end{array}$ & $\begin{array}{l}\text { bei den } 24 \text { Gegen- } \\
\text { seitigkeitsvereinen }\end{array}$ \\
\hline auf gezahlte Schäden ............... & $545 \mathscr{H}$ & $532 \mu$ & $662 \mathscr{M}$ \\
\hline " zurückgestellte Schäden........... & $352 \cdot$ & $366 \cdots$ & 227 \\
\hline - Leistungen für genı. Zwecke ...... & 28 . & 27 . & $31=$ \\
\hline " Zuschnß zur Schadenrücklage ...... & I & 1 & 4 \\
\hline " Zuinahune der I'rämienïberträge . . . & $74 \cdot$ & $74 \cdot$ & $76 \cdot$ \\
\hline
\end{tabular}

Von den im Geschäftsjahr entstandenen Schadensummen wurden durchschnittlich bei den Aktiengesellschaften 59,3 v. H., bei den Gegenscitigkeitsvereinen 74,4 v. H. und bei allen Anstalten zusammen 60,8 v. H. noch im Geschäftsjahre gezahlt. Der Rest wurde der Schadenrücklage überwiesen. - Von den Leistungen für gemeinnützige Zwecke beruhten bei den Aktiengesellschaften 74,6 v. H. und bei den Gegenseitigkeitsvereinen 90,7 v. H. auf gesetzlicher Vorschrift.

An Verwaltungskosten und Steuern hatten für die Feuerversicherung die 40 Aktiengesellschaften $48733622 \mathscr{M}$ und die 24 Gegenseitigkeitsvereine $6996182 \mathscr{M}$ aufzuwenden.

Einen Verlust aus dem Betriebe der Feuerversicherung des Berichtsjahrs von zusammen $294232 \mathscr{N}$ haben 6 Aktiengesellschaften nachgewiesen; den übrigen Gesellschaften hat das Berichtsjahr einen Gewinn von insgesamt $17239814 \mathscr{M}$ gebracht.

Von den Gegenseitigkeitsvereinen hatten zwei einen Verlust, und zwar von $33410 \mathscr{N}$; die übrigen hatten einen Gewinn von insgesamt $24541902 \mathscr{M}$. $\mathrm{Zu}$ dieser Zahl ist jedoch zu bemerken, daß davon allein $17705899 \mathscr{N}$ auf die Gothaer Feuerversicherungsbank und $3544420 \mathscr{A l}$ auf die Württembergische Feuerversicherung kommen. Diese Uberschüsse sind keịn Gewinn im gewöhnlichen. Sinne; sie haben vielmehr darin ihren Grund, daß die genannten Unternehmungen Beiträge erheben, die den voraussichtlichen Bedarf weit übersteigen, und den Uberschuß den Versicherten dann im folgenden Jahre zurückerstatten (vgl. S. 66" und "Entwickelung * S. 50).

Zu der Sturmschäden -, der Wasserleitungsschäden- und der Einbruchdiebstahlversicherung ist hier folgendes zu bemerken:

Es betrug

die Summe der Betriebs-Einnahmen..

die Summe der Betriebs-Eimnahmen.

\begin{tabular}{ccc} 
bei der & bei der Wasserleitungsschäden- \\
$\begin{array}{c}\text { Sturmschäden- } \\
\text { versicherung }\end{array}$ & \multicolumn{2}{c}{ versicherung } \\
$\begin{array}{c}\text { (2 Aktien- } \\
\text { gesellschaften) }\end{array}$ & $\begin{array}{c}\text { (27 Aktien- } \\
\text { gesellschaften) }\end{array}$ & $\begin{array}{c}\text { (1 Geitigkeits- } \\
\text { verein) }\end{array}$
\end{tabular}

35440 Al

13217 . bei der Einbluchdiebstahlversicherung

(43 Aktien- (13 Gegenseitiggesellschaften) keitsvereine)

12670909 in $583230 \mathscr{N}$ 4409690 " $19974 \mathrm{I}$.

Von je $1000 \mathscr{N}$ der Betriebs-Einnahmen kamen:

auf Reinbeiträge ................

- Nebenleistungen der Versicherten

n Ersparnis aus der Schadenrücklage

- Abnahne der Pränienübertrăge .

$\begin{array}{r}929 \\ 1 \\ 4 \\ \hline 1000\end{array}$

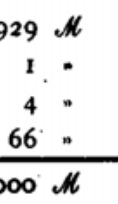

\begin{tabular}{|c|c|c|}
\hline 904 & $\mathscr{M}$ & 979 \\
\hline & $\cdot n$ & $2 \mathrm{I}$ \\
\hline 66 & . & - \\
\hline 25 & . & - \\
\hline
\end{tabular}

\begin{tabular}{|c|c|c|c|}
\hline 820 & $\mathscr{H}$ & 959 & $\mathscr{\mu}$ \\
\hline 12 & . & 20 & - \\
\hline 140 & - & io & - \\
\hline 28 & " & I & " \\
\hline
\end{tabular}

- Von je $1000 \mathscr{N}$ der Betriebs-Ausgaben kamen:

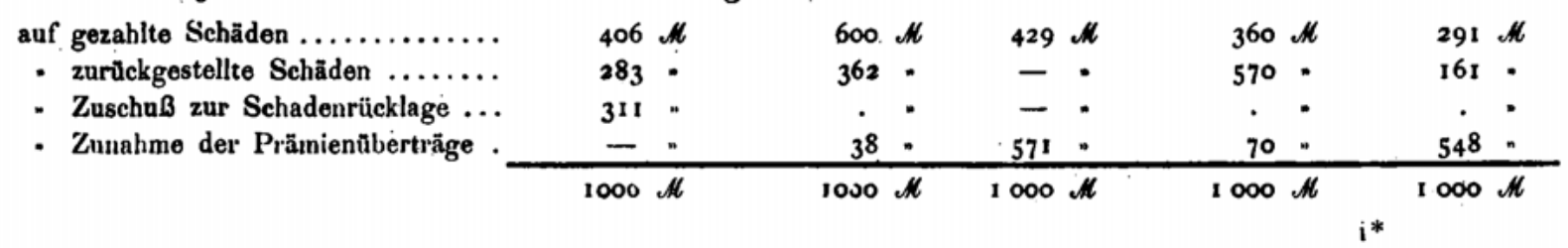


Von den im Geschäftsjahr entstandenen Schäden für eigene Rechnung wurden bei der Sturmschädenversicherung 58,9 v. H., bei der Wasserleitungsschädenversicherung 62,4 v. H. und bei der Einbruchdiebstahlversicherung 38,7 v. H. noch im Geschäftsjahre beglichen, der Rest wurde in Schadenrücklage gestellt.

An Verwaltungskosten und Steuern hat die Sturmschädenversicherung $12937, \mathcal{M}$, die Wasserleitungsschädenversicherung bei den Aktiengesellschaften $956696 \mathscr{M}$, bei 1 Gegenseitigkeitsverein 234 $\mathscr{N}$ und die Versicherung gegen Finbruchdiebstahl bei den Aktiengesellschaften $3762799 \mathscr{M}$ und bei den Gegenseitigkeitsvereinen $151271 \mathscr{N}$ erfordert. In der Sturmschädenversicherung haben beide Aktiengesellschaften einen Gewinn von $9286 \mathscr{M}$. In der Wasserleitungsschädenversicherung haben 6 Aktiengesellschaften einen Verlust von $13622 \mathscr{M}$ und 21 Aktiengesellschaften und 1 Gegenseitigkeitsverein einen Gewinn von $900613 \mathscr{M}$ bezw: $71 \mathscr{M}$ gehabt. In der Einbruchdiebstahlversicherung ist bei den Aktiengesellschaften ein Gewinn von zusammen $4498420 \mathscr{M}$ zu verzeichnen. Das kleine Geschäft der 13 Gegenseitigkeitsvereine hat einen Verlust von $278 \mathscr{M}$ und einen Gewinn von $232496 \mathscr{M}$ gebracht.

Das Ergebnis der Vermõgensverwaltung

Das Ergebnis der Vermögensverwaltung wird von den Unternehmungen in der Regel nicht nach den einzelnen Geschäftszweigen getrennt; nur der Lebensversicherung werden immer bestimmte Erträge überwiesen, weil das der Betrieb dieses Geschäfts erfordert. Es besteht keine Veranlassung, in dieser Statistik von dem Verfahren der Gesellschaften ahzugehen. Soweit also nicht ein Teil des Ertrags einem bestimmten Zweige zugewiesen ist, führen wir das Krgebnis nur insgesamt auf, und zwar im allgemeinen bei derjenigen Gruppe, zu der das Hauptgeschäft der Unternehmung gehört.

In Tafel IV 34 findet sich bei 36 Aktiengesellschaften der, Ertrag der gesamten Vermögensverwaltung, bei 4 Gesellschaften nur der nicht der Lebensversicherung zukommende Teil; der gesamte Ertrag ist für 4 Gesellschaften bei Gruppe II nachgewiesen. Bei den Feuerversicherungs-Gegenseitigkeitsvereinen ist hier überall der Ertrag der gesamten Vermögensverwaltung aufgeführt.

Unter den Erträgen stehen überall die Zinsen im Vordergrund mit $13800106 \mathscr{M}$ bei den Aktiengesellschaften und $3694988 \mathscr{N}$ bei den Gegenseitigkeitsvereinen. Der reine Ertrag der gesamten Vermögensverwaltung beläuft sich dort auf $8736750 \mathscr{M}$ und hier auf $3132984 \mathscr{M}$.

Die Bilanz Eine Bilanz ist in dieser Statistik grundsätzlich nur für das Gesamtgeschäft einer jeden Gesellschaft gegeben und bei der Gruppe aufgeführt, zu der das Unternehmen seinem Hauptbetriebe nach — die Transportversicherung ausgeschlossen - gehört. Dementsprechend sind die Bilanzen der Providentia, Frankfurter Versicherungs-Gesellschaft, und der Bayerischen Versicherungs-Bank bei Gruppe I und der Allianz, des Nordstern Unfall- und Haftpflicht-Versicherungs-Aktien-Gesellschaft, der Frankfurter Allgemeinen Versicherungs-Aktien-Gesellschaft und der Mannheimer Versicherungsgesellschaft bei Gruppe II behandelt, so daß in Tafel IV 38 die Bilanzen nur für 34 deutsche Aktiengesellschaften und 24 Gegenseitigkeitsvereine darzustellen sind. Faßt man diese zusammen, so schließt die Bilanz für die Aktiengesellschaften mit '708143145 $\mathscr{M}$ und für die Gegenseitigkeitsvereine mit $105368593 \mathscr{M}$ ab. Im übrigen mögen folgendè Zahlen hier zusammengestellt werden: 
Vermögenswerte

Verpflichtungen der Aktionäre oder Garaniten.....

Forderungen..$\ldots \ldots \ldots \ldots \ldots \ldots \ldots \ldots \ldots \ldots \ldots$

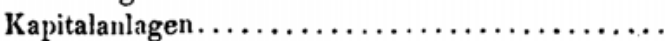

Kassenbestand $\ldots \ldots \ldots \ldots \ldots \ldots \ldots \ldots \ldots \ldots \ldots \ldots \ldots$

Verbindlichkeiten

Aktien- oder Garantiekapital

Gesetzliche Rücklage.

Sonderrücklagen $\ldots \ldots \ldots \ldots \ldots \ldots \ldots \ldots \ldots \ldots$

Guthaben von Versicherungsunternehmungen......

Prämienüberträge $\ldots \ldots \ldots \ldots \ldots \ldots \ldots \ldots \ldots$

Schadenrücklage
34 Altiengesellschaften

$176780100, N$

112762704 .

415307911 .

807422 .
24 Gegenseitigkeitsvereine

851000 it

9581228 ,

93195658 " 451005 *

Die Kapitalanlagen (Tafel IV 39) verteilen sich auf folgende Wertarten:

$\begin{array}{rr}240204080 \mathscr{H} & 2342801 \mathscr{M} \\ 38347011 . & 32692151 " \\ 67412659 " & 5711697 " \\ 45608858 . & 637753 " \\ 188576128 . & 21008764 " \\ 70615152 . & 304822 .\end{array}$

\begin{tabular}{|c|c|c|c|c|c|c|c|}
\hline \multicolumn{4}{|c|}{ bei 34 Aktiengesellschaften } & \multicolumn{4}{|c|}{ bei 24 Gegenseitigkeitsvereincn } \\
\hline $33 \simeq 53254$ & $\mathscr{H}$ oder & 8,0 & H. & 3158612 & $\mathscr{A}$ oder & 3,4 & $\mathrm{H}$ \\
\hline 05518116 &.$\quad$ & 49,5 & " & 36494336 &. & $39, \mathrm{I}$ & n \\
\hline 680674 & - & 0,2 & " & 10991518 & $n$ & 11,8 & - \\
\hline 2747740 & - & 0,7 & ? & - & • & - & $\cdot$ \\
\hline 162593674 & - & 39,1 & * & 41344589 & n & 44,4 & . \\
\hline 6193824 & & $\mathrm{I}, 5$ & $n$ & - & " & - & - \\
\hline I 266252 & $\therefore$ & 0,3 & * & 101602 & • & $0, \mathbf{I}$ & n \\
\hline 3154377 & . & 0,7 & " & 1105001 & $\therefore$ & 1,2 & . \\
\hline
\end{tabular}

Zusanımen .... 415307911 AN oder 100,0 v. H.

$93195658 \AA$ oder 100,0 v. H.

Vergleicht man die Kapitalanlagen der in der Bilanz aufgeführten Untemehmungen am Ende des Berichtsjahrs mit den entsprechenden Anlagen am Ende des Vorjahrs, so findet man eine Zunahme um $3582732 \mathscr{N}$ bei den Hypotheken und Grundschulden, um $36474473 \mathscr{M}$ beim Buchwerte der Wertpapiere (um rund 43 Millionen beim Nennwert) und um $1900510 \mathscr{M}$ bei den sonstigen Anlagen. Zurückgegangen sind der Grundbesitz um $145835 \mathscr{M}$, die Darlehen an öffentliche Körperschaften um $101115 \mathscr{M}$, die Darlehen auf Wertpapiere um $492489 \mathscr{N}$, die Darlehen auf Versicherungen um $11866 \mathscr{M}$ und der Bestand an Wechseln um $224203 \mathscr{N}$.

Die Bilanz weist bei 3 Gegenseitigkeitsvereinen einen Verlust von zusammen $6000 \mathscr{M}$ nach, bei 3 Aktiengesellschaften weder einen Gewinn noch einen Verlust. Alle anderen Unternehmungen haben günstig abgeschlossen. Der bilanzmäßige Gewinn beträgt für die Aktiengesellschaften $27159188 \mathscr{N}$ und für die Gegenseitigkeitsvereine $27840868 \mathscr{M}$; er ist nach Tafel IV 40 wie folgt verteilt worden:

\begin{tabular}{|c|c|c|c|c|c|c|c|c|c|}
\hline \multirow{5}{*}{$\begin{array}{l}\text { an die gesetzliche Rücklage } \ldots \ldots \ldots \ldots \ldots \\
\text { an sonstige Rücklagen } \ldots \ldots \ldots \ldots \ldots \ldots \\
\text { an den Beanitenwohlfahrtsstock } \ldots \ldots \ldots \\
\text { an die Aktionäre (Garanten) } \ldots \ldots \ldots \ldots\end{array}$} & \multicolumn{4}{|c|}{31 Aktiengesellschaften } & \multicolumn{5}{|c|}{20 Gegenseitigkeitsvereine } \\
\hline & 411807 & $\mathscr{M}$ oder & 1,5 & H. & 1125268 & & & 4,0 & \\
\hline & 3431041 & $n \quad n$ & 12,6 & $\cdot$ & 2252994 & 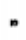 & & 8,1 & " \\
\hline & 597467 & " & 2,2 & n & 444693 & & & $\mathbf{1}, 6$ & n \\
\hline & 14808439 & n & 54,5 & " & 4500 & " & & . & n \\
\hline für Tantiemen.$\ldots \ldots \ldots \ldots \ldots \ldots \ldots \ldots \ldots$ & 2381850 & " & 8,8 & n & 2 I 96 I & n & & $0, \mathrm{I}$ & n \\
\hline an die Versicherten..$\ldots \ldots \ldots \ldots \ldots$. & - & & - & - & 23670068 & " & & 85,0 & $\cdot$ \\
\hline nderweit $\ldots \ldots \ldots \ldots \ldots \ldots \ldots \ldots \ldots \ldots$ & 1865602 & & 6,9 & $n$ & 57951 & $n$ & & 0,2 & $n$ \\
\hline Vortrag auf neue Kechnung...$\ldots \ldots$ & 3662982 & $\cdot$ & 13,5 & * & 263433 & . & n & 1,0 & n \\
\hline
\end{tabular}

Für die ausländischen Gesellschaften sind die Bilanz der Assicurazioni Generali bereits bei der Lebensversicherung in Tafel I 47, die Bilanzen der Ersten Österreichischen Allgemeinen Unfall-Versicherungs-Gesellschaft, der Schweizerischen National-Versicherungs;Gesellschaft, der Schweizerischen Unfallversicherungs-Gesellschaft und der Zürich bei der Unfall- und Haftpflichtversicherung in Tafel II 14 und die Bilanz der 
Union Suisse bei der Glasversicherung in Tafel V 13 gegeben; die Bilanzen der übrigen 9 Unternehmungen finden sich in Tafel IV 42. Im Anschlusse daran "sind in Tafel IV 43 die Kapitalanlagen und die Forderungen erläutert. Wegen des großen Interesses, das der Art der Kapitalanlagen zukommt, sind in der folgenden Ubersicht die Gesellschaften nach Heimatländern zusammengefaßt; zum Vergleiche sind die Verhältniszahlen für die..deutschen Unternehmungen wiederholt.

Von je $1000 \mathscr{M}$ Kapitalanlagen entfielen auf:

\begin{tabular}{|c|c|c|c|c|c|c|c|c|c|}
\hline bei & $\begin{array}{l}\text { Grund- } \\
\text { besitz }\end{array}$ & $\begin{array}{l}\text { Hypo- } \\
\text { theken } \\
\text { und } \\
\text { Grund- } \\
\text { schulden }\end{array}$ & $\begin{array}{c}\text { Darlehen } \\
\text { an öffent- } \\
\text { liche } \\
\text { Körper- } \\
\text { schaften }\end{array}$ & $\begin{array}{c}\text { Darlehen } \\
\text { auf } \\
\text { Wert- } \\
\text { papiere }\end{array}$ & $\begin{array}{l}\text { Wert- } \\
\text { papiere }\end{array}$ & $\begin{array}{l}\text { Darlehen } \\
\text { auf Ver- } \\
\text { siche- } \\
\text { rungen }\end{array}$ & $\begin{array}{l}\text { Wech- } \\
\text { sel }\end{array}$ & $\begin{array}{l}\text { Son- } \\
\text { stige- } \\
\text { An- } \\
\text { lagen }\end{array}$ & $\begin{array}{c}\text { Gesamtbetrag } \\
\text { der }\end{array}$ \\
\hline 1 belgischen Gesellschaft.......... & 69 & - & - & - & 846 & 85 & - & - & $3719933 a$ \\
\hline 1 dänischen Gesellschaft.......... & 232 & 378 & - & - & 390 & - & - & - & 3527096 \\
\hline 2 niederländischen Gesellschaften .. & 159 & 32 & - & - & 603 & 178 & - & 28 & 12065325 . \\
\hline 1 österreichischen Gesellschaft..... & - & $3 \mathbf{I}$ & 15 & - & 927 & 12 & 15 & - & 24650877 . \\
\hline 2 schwedischen Gesellschaften ..... & 43 & 509 & 49 & - & 272 & 127 & - & - & 92601915 * \\
\hline $\begin{array}{c}2 \text { schweizerischen Gesellschaften ... } \\
\text { dagegen bei }\end{array}$ & 119 & 447 & - & - & 421 & - & 13 & - & 19717368 . \\
\hline eutschen Aktiengescllscha & 80 & 495 & 2 & 7 & 391 & 15 & 3 & 7 & $415307911=$ \\
\hline 4 deutschen Gegenseitigkeitsvereinen & 34 & 391 & 118 & - & 444 & - & I & 12 & 93195658 \\
\hline
\end{tabular}

Von den 11 kleinen Vereinen, die in den Tafeln nicht behandelt sind, muß einer wegen unvollständiger Angaben hier ganz ausscheiden, bei den übrigen kommen insgesamt 44410 Versicherungen mit rund 400,85 Millionen Mark in Frage; der durchschnittliche Betrag einer Versicherung stellt sich danach auf $9026 \mathscr{M}$. Die gesamten Einnalımen haben im Jahre $1915397239 \mathscr{N}$ betragen; davon kommen $183614 \mathscr{N}$ oder 46,2 v. H. auf Beiträge, $50377 \mathscr{H}$ oder 12,7 v. $H$. auf Nebenleistungen der Versicherten und $51077 \mathscr{A}$ oder $12,9 . \mathrm{v}$. H. auf Kapitalerträge; von den gesamten Ausgaben in Hölee von $31758 i \mathscr{N}$ kommen $168830 \mathscr{M}$ oder 53,2 v. H. auf Entschädigungen und $77051 \mathscr{K}$ oder 24,2 v. H. auf Virwaltungskosten. Als Vermögen am Schlusse dés Berichtsjahrs haben die 11 Vereine zusammen $1149796 \mathscr{H}$ nachgewiesen.

Diese Ziahlen lassen jedoch kein Urteil über die Bedeutung der kleinen Vereine im Deutschen Reiche zu. Die Zahi der überhaupt vorhandenen kleinen Vereine ist viel größer; auch werden sich unter der Aufsicht der Landesbehörden gewiß mehrere finden, welche die unter Reichsaufsicht stehenden kleinen Vereine an Bedeutung übertreffen.

\section{Gruppe V, Sonstige Versicherungszweige Glasversicherung}

Auf dem Gebiete der Glasversicherung berichtet die Statistik für das Jahr 1915 über 21 deutsche Aktiengesellschaften, 2 deutsche Gegenseitigkeitsvereine und 3 ausländische Gesellschaften. 4 Aktiengesellschaften und 1 Gegenseitigkeitsverein beschäftigten sich ausschließlich mit diesem Versicherungszweige; für die übrigen .Unternehmungen bildet die Glasversicherung nur einen Nebenbetrieb.

Die National Provincial Plate Glass \& General Ins. 'Co. Ltd. in London hat nach Ausbruch des Krieges mit einer deutschen Gesellschaft einen Haftungs- und Ubergangsvertrag abgeschlossen und ist deshalb aus dieser Statistik fortgelassen. 
Uber den Umfang des Geschäfts der deutschen Unternelimungen in der Glasver- Der Umfang sicherung soll Tafel V 1 unterrichten. Da die Versicherungssumme bei eincr Gesellschaft nicht vollständig angegeben werden kann, so empfiehlt sich hier die Beschränkung auf diejenigen Gesellschaften, welche für die Jahre I914 und I9I5 alle Angaben haben des Gemachen können. Bei der Besprechung der Beiträge und der Schäden im Anschluß an Tafel V 4 wird Gelegenheit sein, auf den Geschäftsumfang aller Gesellschaften im Jahre I 9 I 5 zurückzukommen.

An selbst abgeschlossenen Versicherungen bestanden einschließlich der in Rückdeckung gegebenen Beträge:

\begin{tabular}{|c|c|c|c|c|c|c|c|c|c|c|}
\hline \multirow{2}{*}{\multicolumn{2}{|c|}{$\begin{array}{c}\text { bei } 20 \text { Aktiengesellschaften ..... } \\
\text { - } \quad 2 \text { Gegenseitigkeitsvereinen }\end{array}$}} & \multicolumn{5}{|c|}{ Ende 1914} & \multicolumn{4}{|c|}{ Ende 1915} \\
\hline & & $\begin{array}{r}635864 \\
23397 \\
\end{array}$ & $\begin{array}{c}\text { Vers. } \\
.\end{array}$ & $\begin{array}{c}\text { über } \\
:\end{array}$ & $\begin{array}{r}35555^{8} 670 \\
12124596 \\
\end{array}$ & $\stackrel{\mu}{\mu}$ & $\begin{array}{c}629577 \text { Vers. } \\
22805 \text {.. }\end{array}$ & über & $\begin{array}{r}351217340 \\
11826544 \\
\end{array}$ & $\stackrel{n}{*}$ \\
\hline
\end{tabular}

Die durchschnittlich auf eine selbst abgeschlossene Versicherung entfallenden Beträge waren in Mark:

\begin{tabular}{|c|c|c|c|c|c|}
\hline Ende des Jahres & 1911 & 1912 & 1913 & 1914 & 1915 \\
\hline Aktiengesellschaften, Gesamtgeschäft........ & 557 & $5^{62}$ & $5^{62}$ & 562 & 561 \\
\hline inländisches Geschäft $\ldots \ldots \ldots \ldots \ldots \ldots$ & 549 & 555 & 564 & 561 & 559 \\
\hline ausländisches Geschäf̣t $\ldots \ldots \ldots \ldots \ldots \ldots$ & 654 & 639 & 547 & 576 & \\
\hline Gegenseitigkeitsvereine, Gesamtgeschäft ....... & 519 & 520 & 520. & 518 & 519 \\
\hline inlăndisches Gcschåft $\ldots \ldots \ldots \ldots \ldots \ldots$ & 522 & 525 & 524 & 521 & 522 \\
\hline ausländisches Geschäft $\ldots \ldots \ldots \ldots \ldots \ldots$ & 449 & 436 & 447 & $45^{8}$ & 459 \\
\hline Insgesant $\ldots$ & 555 & 560 & 557 & 561 & \\
\hline
\end{tabular}

Außerdem haben die Aktiengesellschaften noch Versicherungen in Höhe von $93169 \mathscr{M}$ am Schlusse des Jahres 1914 und in Höhe von $68673 \mathscr{M}$ am Schlusse von 1915 als in Rückdeckung übernommen nachgewiesen. Der Anteil des mittelbaren Geschäfts am Gesamtgeschäft ist danach ganz unbedeutend gewesen; er stellte sich bei den Aktiengesellschaften im Jahre I914 und im Jahre 1915 auf 0,02 v. H.

Von den selbst abgeschlossenenVersicherungen derjenigen Unternehmungen, bei denen die Zahl der Versicherungen und die Versicherungssummen angegeben sind, kommen:

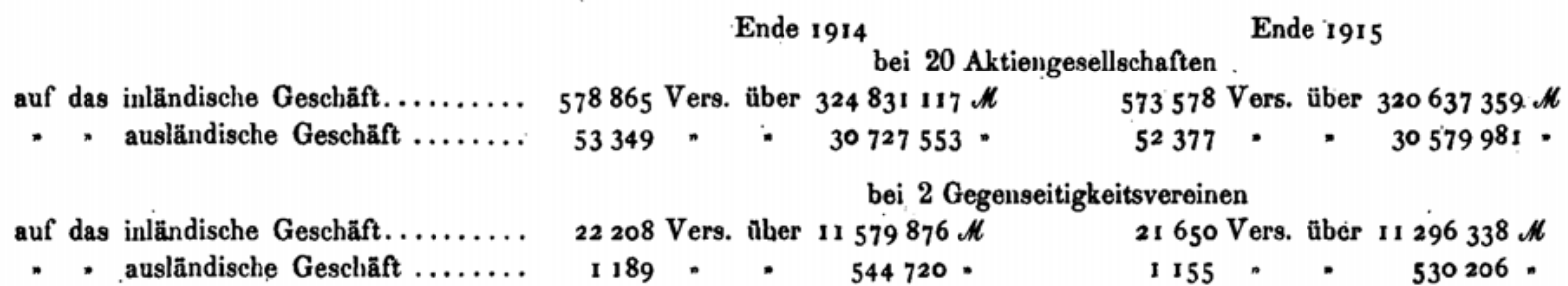

Der Umfang des deutschen Geschäfts der hier eingestellten ausländischen Gesellschaften ist nur ganz unbedeutend (Tafel V 3). Es waren Ende I9 I4 1936 Versicherungen über $850388 \mathscr{M}$ und Fnde I9 I 51 y84 Versicherungen über 921 892 $\mathscr{M}$ in Kraft. Der durchschnittlich auf eine Versicherung kommende Betrag belief sich am Ende des Vorjahrs auf $439 \mathscr{M}$, am Ende des Berichtsjahrs auf $465 \mathscr{M}$. Das Geschäft ist von allen Gesellschaften nur unmittelbar betrieben worden.

Die Einnahme an Rohbeiträgen im Geschäftsjahr und die im Geschäftsjahr entstandenen Schäden sind für die deutschen Unternehmungen in Tafel V 4 und für das deutsche Geschäft der ausländischen Gesellschaften in Tafel V 5 zusammengestellt.

$$
\begin{aligned}
& \text { Dér. } \\
& \text { Umfang des } \\
& \text { deutsehen } \\
& \text { Geschuft: } \\
& \text { der aus- } \\
& \text { landischen } \\
& \text { Unterneh. } \\
& \text { mungen. }
\end{aligned}
$$

Die Roh. beitrăge und Sehsden 
In der Glasversicherung haben im Jahre 19 I 5 die 21 deutschen Aktiengesellschaften $9075668 \mathscr{A}$ und 2 Gegenseitigkeitsvereine $309592 \mathscr{M}$, alle Unternehmungen zusammen also $9385260 \mathscr{M}$. an Beiträgen eingenommen; den Aktiengesellschaften sind Schäden im Betrage von $5896951 \mathscr{M}$, den Gegenseitigkeitsvereinen solche im Betrage von $172027 \mathscr{M}$, zusammen im Betrage von $6068978 \mathscr{M}$ entstanden.

Der durchschnittliche Betrag eines Schadens stellt sich für die Aktiengesellschaften im inländischen Betriebe beim unmittelbaren Geschäft auf $56 \%$, im ausländischen Betrieb auf $28 \mathscr{N}$, für die deutschen Gegenseitigkeitsvereine im Inland auf $53 \mathscr{N}$, im Ausland auf $61 \mathscr{M}$.

Aus dem deutschen Glasversicherungsgeschäfte ('Tafel V 5) haben die 3 ausländischen Gesellschaften im Jahre I9I5 insgesamt $21143 \mathscr{M}$ Rohbeiträge eingenommen; für Schäden hatten sie $10277 \mathscr{M}$, durchschnittlich für einen Schadenfall $73 \mathscr{A}$ bereitzustellen.

Die Ergebnisse des Geschäftsbetriebs sind bei der Glasversicherung, die von den Unternehmungen meist nur als Nebenbetrieb behandelt wird, in derselben Weise dargestellt wie bei der Feuerversicherung (S. 66*). Es ist in Tafel V 6 der Gewinn des Berichtsjahrs allein aus dem Betriebe der Glasversicherung ermittelt, indem die Betriebs-Einnahmen den Betriebs-Ausgaben nebst Verwaltungskosten und Steuern gegenübergestellt sind. Der Gesamtgewinn der Unternehmungen ist in der Bilanz ausgewiesen ('Tafel V 9).

Die Betriebs-Einnahmen sind hier wie bei Gruppe IV die Beiträge für eigene Rechnung, die Nebenleistungen der Versicherten, die Ersparnis aus der Schadenrücklage des Vorjahrs, die Abnahme der Prämienüberträge und außerdem der Erlös aus Bruchglas; als Betriebs-Ausgaben wurden die Schäden des Geschäftsjahrs (nach Abzug des Anteils der' Rückversicherer und der Schadenerstattungen), der Zuschuß zur Schadenrücklage des Vorjalırs und die Zunahme der Prämienüberträge zusammengefaßnt.

Bei den deutschen Unternehmungen betrug in der Glasversicherung:

die Summe der Betriebs-Eimnahmen .........

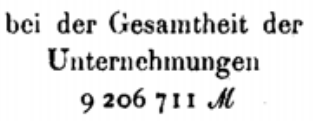

bei 21 Aktiengesellschaften $8888393 \mathscr{H}$ 5431894 . bei 2 Gegenseitigkeitsvereinen 318318, th 182316 ”

Von je 1000 , th der Betriebs-Einnahmen kamen:

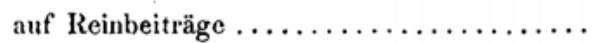

" Nebenleistungen der Versicherten ......

" Ersparnis aus der Schadenrücklage.....

n Abnahme der Prämienüberträge........

- Erlös aus Bruchglas.
$934 \cdot \mathbb{H}$

15 .

25 "

20 .

6 .

I $000 \mathscr{M}$

\begin{tabular}{|c|c|}
\hline $933 \mathscr{M}$ & 967 eth \\
\hline 15. & 17. \\
\hline $25 \%$ & 16 . \\
\hline 21 & $-\cdots$ \\
\hline 6. & - \\
\hline
\end{tabular}

Von je $1000 \mathscr{M}$ der Betriebs-Ausgaben kamen:

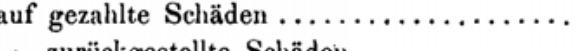

- zurückgestellte Schäden..............

. Zuschuß zur Schadenrücklage.........

n Zunahme der Prämienüberträge.......
$746 \mu$

232 "

2 .

20 "

$000 \mathscr{M}$

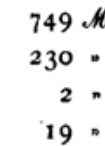

I $000 \mathscr{A H}$
$668 \mathscr{M}$

290 *

-

$42 \cdot$ 
Von den im Geschäftsjahr entstandenen Schadensummen wurden durchschnittlich bei den Aktiengesellschaften 76,5 v. H., bei den Gegenseitigkeitsvereinen $69,7 \mathrm{v}$. H. und bei allen Anstalten zusammen 76,3 v. H. noch im Geschäftsjahre gezahlt; der Rest wurde der Schadenrücklage überwiesen.

An Verwaltungskosten und Steuern haben für die Glasversicherung die 21 Aktiengesellschaften $2794895 \mathscr{A}$ und die Gegenseitigkeitsvereine $108383 \mathscr{M}$ aufgewandt. Aus dem reinen Versicherungsgeschäfte haben 4 Aktiengesellschaften einen Verlust von zusammen $76827 \mathscr{M}$ erlitten und die übrigen einen Gewinn von zusammen $738431 \mathscr{M}$ erzielt; von den Gegenseitigkeitsvereinen hatte der eine einen Gewinn von $28221 \mathscr{M}$ und der andere einen Verlust von $602 \mathrm{H}$.

Das Ergebnis der Vermögensverwaltung ist in Tafel V 7 nur für diejenigen deutschen Unternehmungen zusammengestellt, deren Hauptgeschäft die Glasversicherung ist; das sind 6 Aktiengesellschaften und ein Gegenseitigkeitsverein.

Unter den Erträgen stehen auch hier die Zinsen im Vordergrunde mit 444135 bei den Aktiengesellschaften und mit $40331 \mathscr{M}$ bei dem Gegenseitigkeitsverein. Der reine Ertrag beläuft sich bei den Aktiengesellschaften auf $453327 \mathscr{M}$ und bei dem Gegenseitigkeitsverein auf $44059 \mathscr{H}$.

Die Bilanz der deutschen Unternelımungen ist in 'T'afel $\mathrm{V} 9$ für dieselben $6 \mathrm{Ak}$ - Die Bilans tiengesellschaften und einen Gegenseitigkeitsverein gegeben, die des andern Vereins befindet sich bei Gruppe IV. Sie schließt für Ende I9I5 ab mit $18985108 \not \mathcal{A}$ bei den Aktiengesellschaften und mit $1023577 \mathscr{N}$ bei dem Gegenseitigkeitsverein. Im úbrigen mögen hier folgende Zahlen zusammengestellt werden:

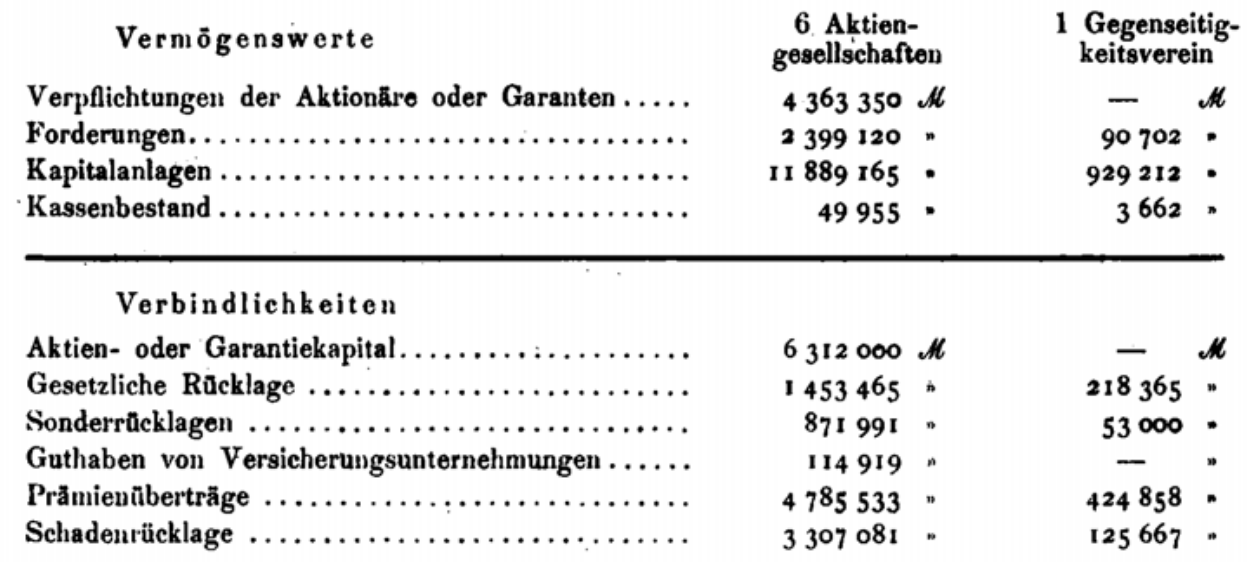

Die Kapitalanlagen ('Tafel V 10) verteilen sich auf folgende Wertarten:

\begin{tabular}{|c|c|c|c|c|c|c|}
\hline \multirow{2}{*}{$\dot{\text { Grundbesitz } \ldots \ldots \ldots \ldots \ldots \ldots \ldots \ldots \ldots}$} & \multicolumn{3}{|c|}{$\begin{array}{l}\text { bei } 6 \text { Aktien- } \\
\text { gesellschaften }\end{array}$} & \multicolumn{3}{|c|}{$\begin{array}{l}\text { 'bei } 1 \text { Gegenseitig- } \\
\text { keitsverein }\end{array}$} \\
\hline & 1423500 & $\mathscr{N}$ oder & $12,0 \%$ & 35500 & $\mathscr{A}$ oder & $3,8 \%$ \\
\hline Hypotheken und Grundschulden ....... & 8395538 & $\cdot \quad "$ & $70,6 \cdots$ & 687500 & $n \quad$, & 74,0 \\
\hline Darlehen an öffentliche Körperschaften... & - & . & - & - & . & $\rightarrow$ \\
\hline Darlehen auf Wertpapiere............. & 9813 & $\cdot$ & $0,1 \quad n$ & - & n & - \\
\hline 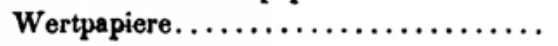 & 2060314 & * & 17,3 * & 206212 & $n$ & 22,2 \\
\hline Wechsel $\ldots \ldots \ldots \ldots \ldots \ldots \ldots \ldots \ldots \ldots$ & - & $n$ & - & 一 & n & - \\
\hline
\end{tabular}


Die Kapitalanlagen sämtlicher 7 Unternehmungen haben Ende 1915 insgesamt $821202 \mathscr{M}$ mehr betragen als am Schlusse des Vorjahrs. Zugenommen haben der Hypothekenbestand um $103001 \mathscr{N}$ und die Wertpapiere um $769208 \mathscr{M}$; dagegen sind der Grundbesitz um $47193 \mathscr{M}$, die Darlehen auf Wertpapiere um $3077 \mathscr{H}$ und der Bestand an Wechseln um $737 \mathscr{M}$ zurückgegangen.

Der bilanzmäßige Gewinn ist nach Tafel V 11 wie folgt verteilt worden:

\begin{tabular}{|c|c|c|c|c|c|c|c|c|}
\hline \multirow{9}{*}{$\begin{array}{l}\text { an die gesetzliche Rūcklage } \ldots \ldots \ldots \ldots \ldots \\
\text { an sonstige Rücklagen } \ldots \ldots \ldots \ldots \ldots \ldots \\
\text { an den Beamtenwohlfahrtsstock } \ldots \ldots \ldots \\
\text { an die Aktionäre (Garanten) } \ldots \ldots \ldots \ldots \ldots \\
\text { für Tantiemen } \ldots \ldots \ldots \ldots \ldots \ldots \ldots \ldots \\
\text { an die Versicherten } \ldots \ldots \ldots \ldots \ldots \ldots \ldots \\
\text { anderweit } \ldots \ldots \ldots \ldots \ldots \ldots \ldots \ldots \\
\text { Vortrag auf neue Rechnung } \ldots \ldots \ldots \ldots \ldots\end{array}$} & \multicolumn{4}{|c|}{$\begin{array}{l}\text { bei } 6 \text { Aktien- } \\
\text { gesellschaften }\end{array}$} & \multicolumn{4}{|c|}{$\begin{array}{l}\text { bei } 1 \text { Gegenseitig- } \\
\text { keitsverein }\end{array}$} \\
\hline & 36355 & $\mathscr{N}$ oder & $3,7 \mathrm{v}$ & H. & I 314 & $\mathscr{N}$ oder & 2,5 & H. \\
\hline & 299880 & n $=$ & 30,8 & . & - & $n \quad$ & - & • \\
\hline & - & . & - & n & - & : & - & n \\
\hline & 374920 & " & $3^{8,5}$ & - & - & $n$ & - & - \\
\hline & 160980 & " & 16,5 & $"$ & 21019 & $n$ & 40,0 & $n$ \\
\hline & - & " & - & $n$ & 30214 & * & 57,5 & " \\
\hline & 39479 & n & 4,1 & $n$ & - & $n$ & - & $n$ \\
\hline & 62276 & $n$ & 6,4 & . & - & $"$ & - & n \\
\hline
\end{tabular}

Die gesetzliche Rücklage steht in der diesjährigen Bilanz bei den Aktiengesellschaften um $34761 \mathscr{N}$ und bei dem Gegenseitigkeitsverein um $17676 \mathscr{M}$ höher als in der vorjährigen Bilanz. Die Sonderrücklagen sind bei den Aktiengesellschaften um $255391 \mathscr{M}$ gesunken, bei dem Gegenseitigkeitsverein um $19000 \mathscr{M}$ gewachsen.

Für die ausländischen Gesellschaften ist die Bilanz der Assicurazioni Generali bei der Lebensversicherung in Tafel I 47 und die Bilanz der Schweizerischen NationalVersicherungs-Gesellschaft in Tafel II 14 gegeben; die Bilanz der andern Unternehmung findet sich in Tafel V 13. Im Anschlusse daran sind in Tafel V 14 die Kapitalanlagen und die Forderungen erläutert.

\section{Kautions- und Bürgschaftsversichèrung}

Dieser Versicherungszweig wird nur nebenher betrieben. Auf das kleine Geschäft, das der Atlas in Ludwigshafen, die Wilhelma in Magdeburg und die Bayerische Versicherungs-Bạnk in München auf dem Gebiete der Kautionsversicherung erledigt haben, braucht hier nicht eingegangen zu werden (vgl. die Anmerkungen 4, 5 und 6 zu 'Tafel I 30 S. 92 u. 93). Von ausländischen Gesellschaften waren zum Betriebe dieses Zweiges im Deutschen Reiche befugt die Schweizerische Unfallversicherungs-Aktiengesellschaft in Winterthur und die Zürich, Allgemeine Unfall- und Haftpflicht-Versicherungs-Aktiengesesellschaft in Zürich.

Uber den Umfang des Geschäfts der hier behandelten 3 deutschen Aktiengesellschaften und seine Zusammensetzung aus unmittelbaren und mittelbaren Abschlüssen sowie über den Anteil des inländjschen Geschäfts am Gesamtgeschäfte gibt Tafel V 15 Aufschluß. Danach liefen bei diesen Gesellschaften insgesamt an selbst abgeschlossenen Versicherungen einschließlich der in Rückdeckung gegebenen Beträge:

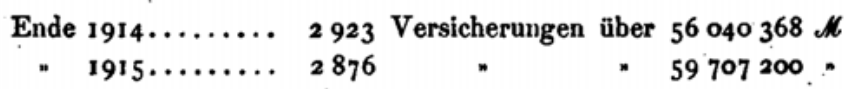

so daß die Zahl der Versicherungen um 47 ab- und die versicherten Summen um $3666832 \mathscr{M}$ zugenommen haben: Außerdem hatten die Gesellschaften Ende 19 i 5 noch $118602873 \mathscr{M}$ gegen $110270841 \mathscr{M}$ am Ende des Vorjahrs in Rückdeckung übernommen. 
Von dem gesamten übernommenen Betrage von $178310073 \mathscr{M}$ liefen $89058921 \mathscr{H}$ für eigene Rechnung.

Von den selbst abgeschlossenen Versicherungen kamen:

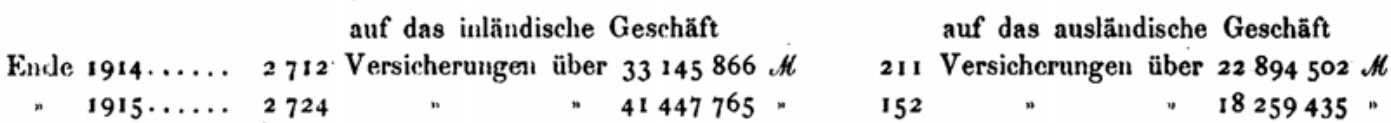

- Die Rohbeitragseinnahme hat nach Tafel V 17 im Jahre $1915993063 . \mathcal{H}$ betragen; davon kommen $909142 \mathscr{H}$ auf das inländische und $83921 \mathscr{H}$ auf das ausländische Geschäft. Von den überhaupt entstandenen Schadensummen in Höhe von $686640 \mathscr{M}$ kommen $653148 \mathscr{M}$ auf das Inland und $33492 \mathscr{M}$ auf das Ausland.

Die Betriebs-Ëinnahmen betrugen $835364 \mathscr{M}$, während die Betriebs-Ausgaben $712329 \mathscr{M}$ ausmachten; an Verwaltungskosten und Steuern sind $157670 \mathscr{M}$ aufgewandt worden. Der Betriebsgewinn aus dem Kautions- und Bürgschaftsversicherungsgeschäfte hat sich für 2 Gesellschaften zusammen auf $13444 \mathscr{N}$ belaufen, eine Gesellschaft hat einen Verlust von $48079 \mathscr{M}$.

Die beiden ausländischen Gesellschaften ('Tafel V 16) hatten Ende 19 I 5 in Deutschland zusammen $10806540 \mathscr{M}$ versichert, gegenüber $13962380 \mathscr{M}$ am Ende des Vorjahrs; sie haben im Berichtsjahre $113373 \mathscr{M}$ an Beiträgen eingenommen und $115891 \mathscr{M}$ für Schäden vergütet.

\section{Kreditversicherung}

Die Kreditversicherung ist nur von geringer Bedeutung. Sie wird bei uns von 2 deutschen Aktiengesellschaften betrieben. Von dem Kreditversicherungsgeschäfte einer ausländischen G'esellschaft kann hier wegen seiner Geringfügigkeit abgesehen werden; es genügt, kurz über das Geschäft der deutschen Unternehmungen zu berichten.

Vom unmittelbaren Geschäfte waren in Kraft:

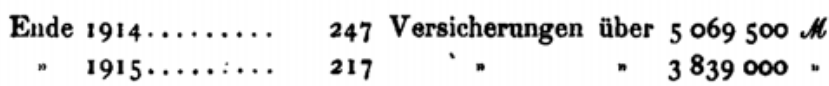

Es hat also die Zahl der Versicherungen um 30 und die versicherte Summe um $1230500 \mathscr{M}$ abgenommen. In Rückdeckung übernommen waren am Ende 1915 $716680 \mathscr{M}$.

Der weitaus größte Teil der überhaupt übernommenen Summen wurde in Rückdeckung weitergegeben, von 4,55 Millionen Mark blicben nur 1,48 Millionen für eigene Rechnung.

Das unmittelbare Geschäft stammt fast vollständig aus dem Deutschen Reiche.-

Dic Rohbeitragseinnahme hat nach Tafel V 17 im Jahre 1915 $410530 \mathscr{M}$ betragen; davon kommen $291742 \mathscr{M}$ auf das inländische und $118788 \mathscr{M}$ auf das ausländische Geschäft. Von den Schäden in einem Gesamtbetrage von $315992 \mathscr{M}$ entfallen $261673 \mathscr{M}$ auf den inländischen und $54319 \mathscr{M}$ auf den ausländischen Betrieb.

Die Betriebs-Einnahmen betrugen $186490 \mathscr{M}$, während die Betriebs-Ausgaben $126803 \mathscr{M}$ ausmachten; für Verwaltungskosten und Steuern sind $46298 \mathscr{M}$ aufgewandt worden. Eine Gesellschaft hatte aus dem Kreditversicherungsgeschäft einen Gewinn von $13389 \mathscr{N}$ gehabt. 


\section{Maschinenversicherung}

Die Maschinenversicherung ist in Jahre 1915 bei uns von 3 deutschen Aktiengesellschaften und in geringem Umfange von einer ausländischen Gesellschaft betrieben worden. Es genügt hier, nur den Geschäftsumfang der 3 deutschen Gesellschaften kurz zu erwähnen. Das Geschäft war fast ganz auf das Deutsche Reich beschränkt.

Nach Tafel V 15 waren an selbst abgeschlossenen Versicherungen in Kraft:

$$
\begin{aligned}
& \text { Ende } 1914 \ldots \ldots \ldots .2332 \text { Versicherungen über } 351504266 \mathscr{A} \\
& \text { " 1915...... } 2268 \text { " } 393025640 \text { n }
\end{aligned}
$$

Im Berichtsjahre hat also die Zahl der Versicherungen um 64 abgenommen und die Versicherungssumme um $41521374 \mathscr{M}$ zugenommen.

Von diesen Versicherungen kamen:

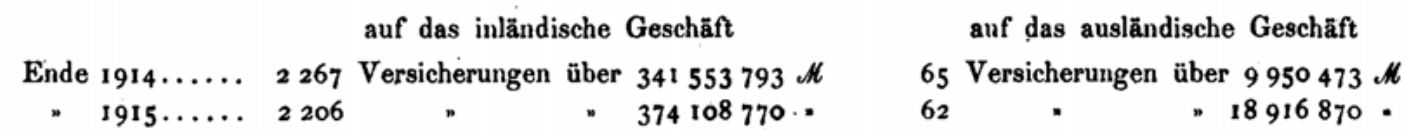

In der Maschinenversicherung läuft nur ein verhältnismäßig kleiner 'Teil für eigene Rechnung: von 415,11 Millionen Mark 118,00 Millionen.

Die Rohbeitragseinnahme hat nach Tafel V 17 im Jahre.1915 $839614 \mathscr{M}$ betragen; davon entfallen auf das inländische Geschäft $764923 \mathscr{M}$, auf das ausländische Geschäft $74691 \mathscr{M}$. Für Schäden sind $576729 \mathscr{M}$ gezahlt worden; davon betreffen $553604 \mathscr{N}$ inländische und $23125 \mathscr{N}$ ausländische Schadenfälle.

Die Betriebs-Einnahmen haben $307358 \mathscr{M}$ betragen, während die Betriebs-Ausgaben $192586 \mathscr{M}$ ausmachten; für Verwaltungskosten und Steuern sind $58182 \mathscr{M}$ aufgewandt worden. Das Maschinenversicherungsgeschäft hat einen Gewinn von insgesamt $56590 \mathscr{M}$ gebracht.

\section{Baulastversicherung}

Die Baulastversicherung ist im Jahre 1915 nur unmittelbar und nur innerhalb des Deutschen Reichs von einem Gegenseitigkeitsverein betrieben worden.

Nach Tafel V 15 waren an selbst abgeschlossenen Versicherungen in Kraft:

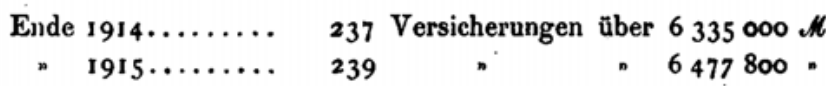

Es hat also im Berichtsjahre die Zahl der Versicherungen um 2 und die Versicherungssumme um $142800 \mathscr{M}$ zugenommen.

Von den übernommenen Versicherungen ist nichts in Rückdeckung gegeben worden.

Die Rohbeitragseinnahme hat nach Tafel V 17 im Jahre I9I5 $27350 \mathscr{M}$ betragen; für Schäden sind $38418 \mathscr{M}$ gezahlt worden.

Die Betriebs-Einnahmen haben $27379 \mathscr{M}$ betragen; demgegenüber stehen die Betriebs-Ausgaben mit $39040 \mathscr{M}$ und die Verwaltungskosten und Steuern mit $2176 \mathscr{M}$, so daß ein Verlust von $13837 \mathscr{M}$ zu verzeichnen ist. 


\section{Wertgegenständeversicherung}

Diese Versicherungsart ist auch im Berichtsjahre nur von einer Aktiengesellschaft betrieben worden.

Fs wurden nur unmittelbare Versicherungen (nur im Inlande) abgeschlossen. Das Geschäft umfaßte am Ende des Berichtsjahrs 429) Versicherungen über $6395312 \mathscr{M}$ gegenüber 449 Versicherungen über $5718292 \mathscr{N}$ am Ende des Vorjahrs. Für eigene Rechnung wurden nur $2890633 \mathscr{H}$ behalten. Die Beitragseinnahme belief sich auf $21291 \mathscr{N}$; für Schäden waren $8008 \leadsto$ zu vergütein.

Die Betriebs-Einnahmen haben $10333 \mathscr{M}$ betragen, während die Betriebs-Ausgaben $4817 \mathscr{H}$ ausmachten; für Verwaltungskosten und Steuern sind $5254 \mathscr{M}$ aufgewandt worden. Das Wertgegenständeversicherungsgeschäft hat demnach einen Gewinn von $262 \mathscr{M}$ ergeben.

\section{Veruntreuungsversicherung}

Die Veruntreuungsversicherung wurde auch im Berichtsjahre nur von einer Aktiengesellschaft und von dieser auch nur nebenher betrieben.

Nach Tafel V 15 waren an selbst abgeschlossenen Versicherungen in Kraft:

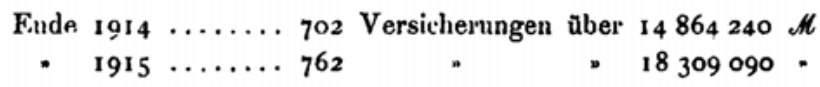

In Rückdeckung übernommen waren am Ende 1915 $538619 \mathscr{M}$ aus dem Auslande. Es hat also im Berichtsjahre die Zahl der Versicherungen um 60 und die Versicherungssumme um $3444850 \mathscr{N}$ zugenommen. Von dem gesamten übernommenen Betrage von $18847709 \mathscr{N}$ liefen $9501928 \varkappa$ für eigene Rechnung.

Die Rohbeitragseinnahme hat nach Tafel V 17 im Jahre I9I $5139273 \mathscr{M}$ betragen; für Schäden sind $36046 \mathscr{M}$ gezahlt worden.

Die Betriebs-Einnahmen betrugen $55885 \mathscr{M}$, während die Betriebs-Ausgaben $21532 \mathscr{M}$ ausmachten; an Verwaltungskosten und Steuern sind $21161 \mathscr{M}$ aufgewandt worden.

Das Veruntreuungsversicherungsgeschäft hat demnach einen Gewinn von $13192 \mathscr{M}$. ergeben.

\section{Hypothekenversicherung}

Die Hypothekenversicherung ist im Berichtsjahre nur unmittelbar und nur innerhalb des Deutschen Reichs von einer Aktiengesellschaft betrieben worden.

Nach 'Tafel V 15 umfaßte das Geschäft am Ende des Berichtsjahrs 30 Versicherungen über $9070380 \mathscr{A}$ wie im Vorjahre. Für eigene Rechnung wurden nur $10 \mathrm{v} . \mathrm{H}$. des versicherten Betrags behalten.

Die Rohbeitragseinnahme hat nach Tafel V 17 im Jahre 1915 $18896 \mathscr{M}$ betragen; für Schäden sind $62471 \mathscr{M}$ gezahlt worden. Die Betriebs-Einnahmen haben $7549 \mathscr{H}$ betragen; demgegenüber stehen die Betriebs-Ausgaben mit $62957 \mathscr{M}$ und die Verwaltungskosten und Steuern mit $2059 \mathscr{M}$, so daß ein Verlust von $57467 \mathscr{M}$ zu verzeichnen ist. 


\section{Stellenlosenversicherung}

Die Stellenlosenversicherung wurde im Berichtsjahre unter Reichsaufsicht nur von zwei kaufimännischen Vereinigungen für ihre Mitglieder als Ergänzung ihrẹ Stellenvermittlung betrieben, und zwar oline Rückversicherung.

Das Geschäft war bisher nicht belangrcich: Ende 1914 waren 136325 und Ende 1915 136178 Vereinsmitglieder versichert; in Laufe des Berichtsjahrs wurden an 693 Mitglieder Entschädigungen gezahlt. Die Betriebs-Einnahmen wiesen für das. Berichtsjahr $135005 \mathscr{N}$ Beiträge auf. Als Stellenlosenunterstützung wurden $130777 \mathscr{M}$ ausgezahlt. Die Verwaltungskosten betrugen $49766^{\circ} \mathscr{M}$.

\section{Automobilkaskoversicherung}

Die Automobilkaskoversicherung (gegen eigene Beschädigung der Wagen) ist im Berichtsjahre von der Rheinischen Feuerversicherungs-Aktiengesellschaft Cöln und vorn Kronprinz, Versicherungs-Aktiengesellschaft Cöln. nur innerhalb des Deutschen Reichs betrieben worden.

Nach Tafel V 15 waren an selbst. abgeschlossenen Versicherungen in Kraft:

58 Versicherungen über $579650 \mathscr{M}$ :

In Rückdeckung übernommen waren am Ende 1915 $144913 \mu$.

Von dem gesamten übernommenen. Betrage vol $724563 \mathscr{M}$ liefen $289825 \mathscr{M}$ für eigene Rechnung.

Die Rohbeitragseinnahme hat nach Tafel V 17 im Jahre I9I5 $10819 \mathscr{M}$ betragen; für Schäden sind $8005 \mathscr{M}$ gezahlt worden.

Die Betriebs-Einnahmen haben $7565 \mathscr{M}$ betragen; demgegenüber stehen die Betriebs-Ausgaben mit $12680 \mathscr{M}$ und die Verwaltungskosten und Steuern mit $601 \mathscr{M}$, so daß ein Verlust von $5716 \mathscr{M}$ zu verzeichnen ist.

\section{Fliegerschädenversicherung.}

Die Fliegerschädenversicherung ist im Berichtsjahre von 2 Aktiengesellschaften, Allianz, Versicherungs-Aktien-Gesellschaft in Berlin und Stuttgart-Berliner VersicherungsAktiengesellschaft in Stuttgart und von einem Gegenseitigkeitsvereine, Württembergische Feuerversicherung auf Gegenseitigkeit in Stuttgart eingeführt worden.

Nach Tafel V 15 waren an selbst abgeschlossenen Versicherungen Ende 19I5 in Kraft:

bei 2 Aktiengesellschaften ... 2388 Vers. über 207671596 . H

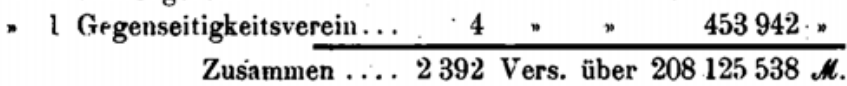

In Rückdeckung übernommen waren bei einer Aktiengesellschaft am Ende I9 I5 $65281530 \mathscr{M}$.

Die Rohbeitragseinnahme hat nach Tafel V 17 im Jahre I9I5 bei den Aktiengesellschaften $613783 \mathscr{M}$ und beim Gegenseitigkeitsvereine $307 \mathscr{M}$ betragen; für Schäden sind bei einer Aktiengesellschaft $604095 \mathscr{M}$ gezahlt worden. 


\section{Kaskoversicherung}

Die Kaskoversicherung wurde im Jahre 19 5 unter Reichsaufsicht von 37 deutschen Unternehmungen betrieben. Davon waren 34 Gegenseitigkeitsvereine und 3 eingetragene Genossenschaften.

Nach Tafel V 20 waren von diesen Unternehmungen versichert:

$$
\begin{aligned}
& \text { Ende r914 } \ldots \ldots \ldots .874 \text { Fahrzenge mit } 103218412 \mu \\
& \text { " } 1915 \ldots \ldots \ldots 88364 \text { " } 88193980 \text { " }
\end{aligned}
$$

Im Berichtsjahre hat die Zahl der versicherten Fahrzeuge um $37 \overline{7}$ und die Versicherungssumme um $5024432 \mathscr{N}$ abgenommen.

Die Summe der Einnahmen aller 37 Vereine hat im Jahre I9I51299581 $\mathcal{N}$ betragen, während die Summe der Ausgaben $2347006 \mathscr{M}$ ausmachte. 19) Vereine haben einen Uberschuß von $64893 \mathscr{M}$ erzielt; 15 Vereine haben einen Verlust von $112318 \mathscr{M}$ erlitten ('Tafel V 21).

Das Vermögen der Unternehmungen betrug Ende I914 $5364418 \mathscr{A}$ und Ende $19155560689 \mathscr{M}$; es hat also um $196271 \mathscr{M}$ zugenommen und bestand Ende 19 5 zu 14,6 v. H. in Sparkasseneinlagen, zu $(2,1$ v. H. in Wertpapieren, zu 9,0 v. H. in Hypotheken, zu 0,9 v. H. in barer Kasse und zu 13,4 v. H. in anderen Werten.

\section{Rilckversicherung}

Von den deutschen privaten Versicherungsunternehmungen, welche die Rückversicherung in gesetzlich aufsichtspflichtigen Versicherungszweigen zum Gegenstande haben, sind für das Jahr I9 15 bei dieser Gruppe 35 behandelt, die in solchen Zweigen lediglich mittelbar arbeiten, und außerdem noch 5, die in einigen Zweigen unmittelbare und mittelbare, in andern nur mittelbare Geschäfte abschließen. Bei diesen letzteren Unternehmungen werden hier aber neben etwaiger 'Transportversicherung nur diejenigen aufsichtspflichtigen $Z$ weige dargestellt, die nur nittelbar betrieben werden; die übrigen sind bereits in den vorhergehenden Gruppen behandelt. $\mathrm{Zu}$ den lediglich mittelbar arbeitenden Unternehmungen sind im Geschäftsjahre die Düsseldorfer Allgemeine VersicherungsAktien-Gesellschaft in DüsseIdorf und die Continentale Versicherungs-Gèsellschaft in Mannheim hinzugekommen.

Sämtliche Unternehmungen sind Aktiengesellschaften.

Ausländische Versicherungsunternehmungen der in Rede stehenden Art unterstehen nicht der Aufsicht des Amts und kommen daher hier nicht in Betracht.

Versicherungssummen, durch die sonst zumeist der Geschäftsumfang gemessen wird, sind die Gesellschaften nur bei der Lebensversicherung anzugeben in der Lage; bei den andern Zweigen können nur die Beiträge und die Schäden herangezogen werden. Unter diesen Umständen scheint eine besondere Behandlung der Versicherungssummen nicht angezeigt; es wird genügen, hier die Beiträge und Schäden nach Versicherungszweigen zusammenzustellen ('Tafel V 24): 


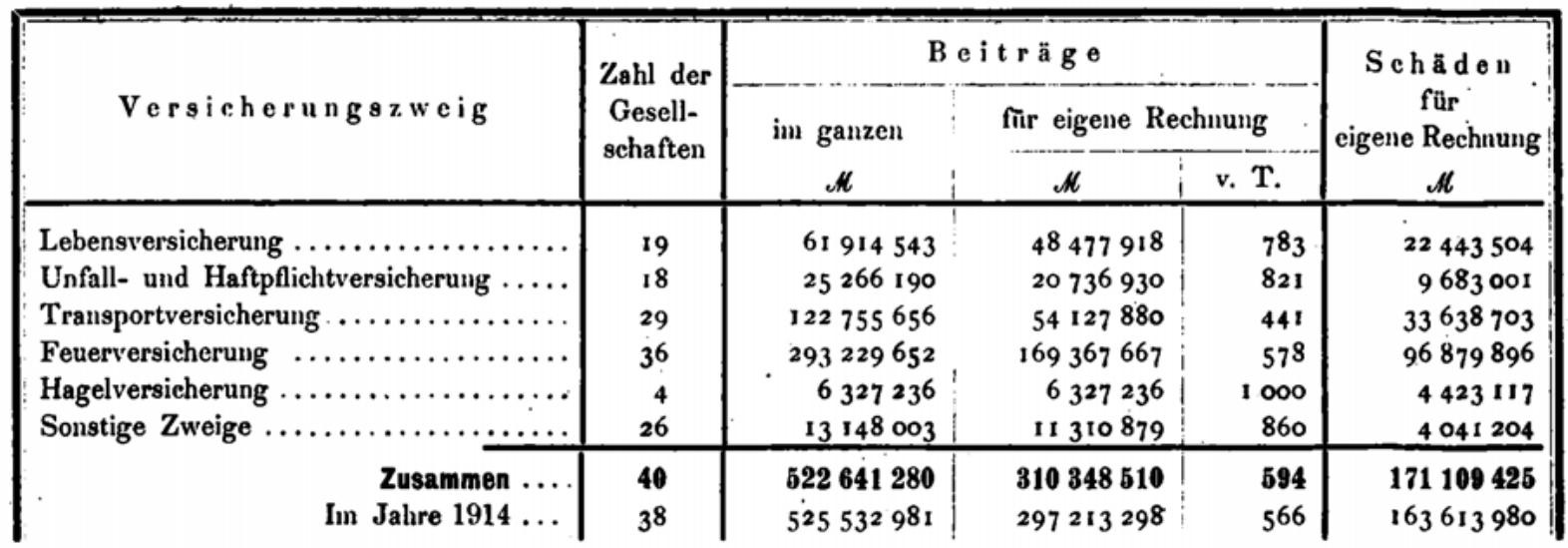

Die Gewinn- und Verlustrechnung (Tafel V 23) bezieht sich auf den gesamten Geschäftsbetrieb der Unternehmungen; nur bei den bereits obenerwähnten fünf Gesellschaften, die außer der Rückversicherung auch das unmittelbare Geschäft in aufsichtspflichtigen Zweigen betreiben, sind hier neben der Transportversicherung nur diejenigen Zweige eingestellt, in denen sie lediglich mittelbar tätig sind. Wie sich das gesamte Geschäft aus den einzelnen Zweigen aufbaut, ist aus den bereits aus Tafel V 24 mitgeteilten Zahlen zu ersehen. Hier bleibt nur noch folgendes zu erwähnen.

Die Vermögenserträge beliefen sich insgesamt im Reinergebnis auf $17891112 \mathscr{M}$; einem Kursgewinne von $1345754 \mathscr{M}$ standen $5987723 \mathscr{M}$ an Kursverlusten gegenüber. Von den Rücklagen haben nach Abzug der Abnahmen die Prämienreserven und Uberträge um $29800700 \mathscr{M}$, die Schadenrücklagen um $23807498 \mathscr{M}$ und die sonstigen Rïcklagen um 488586 $\mathscr{M}$ zugenommen. Die Verwaltungskosten und Steuern haben $89700724 \mathscr{M}$ betragen.

Von den 40 Gesellschaften haben zwei die Ausgaben mit den Einnahmen abgeglichen, eine hat einen Verlust von $2395 \mathscr{M}$ nachzuweisen gehabt; die übrigen haben einen Gewinn von zusammen $12309637 \mathscr{M}$ und mit Einbeziehung des Gewinnvortrags aus dem Vorjahre von $18718602 \mathscr{M}$ erzielt.

Die Bilanx

Die Bilanzen der mehrfach erwähnten fünf Gesellschaften, welche aufsichtspflichtige Versicherungszweige auch unmittelbar betreiben, sind bereits bei Gruppe II in Tafel II 6 (Cölnische Unfall-Versicherung-Aktien-Gesellschaft, Frankfurter Allgemeine Versicherungs-Aktien-Gesellschaft und Oberrheinische Versicherungs-Gesellschaft), oder bei Gruppe IV in Tafel IV 38 (Iduna und Stuttgart-Berliner Versicherungs - Aktiengesellschaft) nachgewiesen; es blieben hier also nur noch die Bilanzen für 35 Gesellschaften zusammenzustellen, was in Tafel V 25 geschehen ist.

Aus dieser Tafel sind folgende Zahlen zu entnehmen:

35 Aktiengesellschaften

Vermögenswerte

Verpflichtungen der Aktionäre ...... $115375137 \mathscr{M}$

Forderungen................. 187621700 .

Kapitalanlagen................ 249561911 .

Kassenbestand ............ $\quad 172650$.
Verbindlichkeiten.

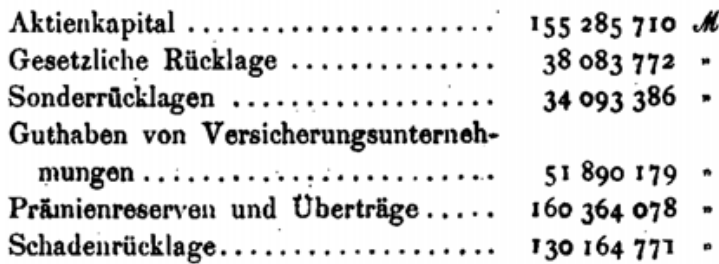


Die Kapitalanlagen verteilen sich auf folgende Wertarten (Tafel V 26): $8007090 \mathscr{M}$ oder 3,2 v. H. Grundbesitz, $55199164 \mathscr{N}$ oder 22,1 v. H. Hypotheken, $183562647 \mathscr{N}$ oder 73,6 v. H. Wertpapiere, $464862 \mathscr{M}$ oder 0,2 v. H. Wechsel, $1515480 \mathscr{M}$ oder 0,6 v. H. Darlehen an öffentliche Körperschaften, $627653 \mathscr{M}$ oder 0,2 Darlehen auf Wertpapiere und $185015 \mathscr{M}$ oder 0,1 v. H. sonstige Anlagen.

Uber die Wertpapiere mag folgendes mitgeteilt werden: Dem Nennwerte nach handelt es sich bei den betrachteten 35 Gesellschaften (von denen indessen eine überhaupt keine Wertpapiere im Besitz hatte) um insgesamt $222205652 \mathscr{M}$, wovon $126572500 \mathscr{M}$ oder 57,0 v. H. auf inländische und $95633152 \mathscr{A}$ oder 43,0 v. H. auf ausländische Werte kommen. Die deutschen Anleihen bestehen aus $80102800 \mathscr{M}$. (36,1 v. H. aller Wertpapiere) Reichs- oder Staatsanleihen, $354500 \mathscr{M}(0,2$ v. H.) staatlich gedeckten Anleihen, 9329800 At (4,2 v. H.) anderen öffentlichen Anleihen, 10293000 A (4,6 v. H.) Pfandbriefen, 2764900 N (1,2 v. H.) Obligationen und $1480500 \mathscr{H}(0,7$ v. H.) Aktien von Banken, Eisenbahn- und sonstigen industriellen Unternehmungen, 22234500 th (10,0 v. H.) Aktien von Versicherungsunternehmungen und $12500 \mathscr{N}(0,006$ v. H.) sonstigen Wertpapieren. Die ausländischen Anlagen bestehen aus $40406910 \mathscr{M}(18,2$ v. H. aller Wertpapiere) Staats-, staatlich gedeckten und andern öffentlichen Anleihen, $30027763 \mathscr{A}(13,5 \mathrm{v}$. H.) sonstigen Schuldverschreibungen und 25198479 eth (11,3 v. H.) Aktien.

Als Prämienreserven und Uberträge sind, um die Ubbereinstimmung mit den Bilanzen der andern Gruppen zu wahren, auch hier nur die in eigener Verwahrung und Verwaltung der betrachteten Unternehmungen befindlichen Beträge in die Bilanz eingestellt worden. Tafel V 27 zeigt, wie diese Beträge ermittelt wurden.

Der bilanzmäßige Gewinn dieser 35 Gesellschaften beträgt $17738226 \mathscr{H}$; er ist nach Tafel V 28 wie folgt verteilt worden: an die gesetzliche Rücklage $462401 \mathscr{M}$ oder 2,6 v. H., an die sonstigen Rücklagen $1022165 \mathscr{H}$ oder 5,8 v. H., an den Beamtenwohlfahrtsstock $89639 \mathscr{M}$ oder 0,5 v. H., an die Aktionäre $8214885 \mathscr{N}$ oder 46,3 v. H., als Tantiemen $880064 \mathscr{M}$ oder 5,0 v. H., als Vortrag auf neue Rechnung $6395873 \mathscr{M}$ oder 36,0 v. H. und endlich anderweit $673199 \mathscr{M}$ oder 3,8 v. H. 



\section{Tafel}

zur

Versicherungs-Statistik des Aufsichtsamts für 1915 

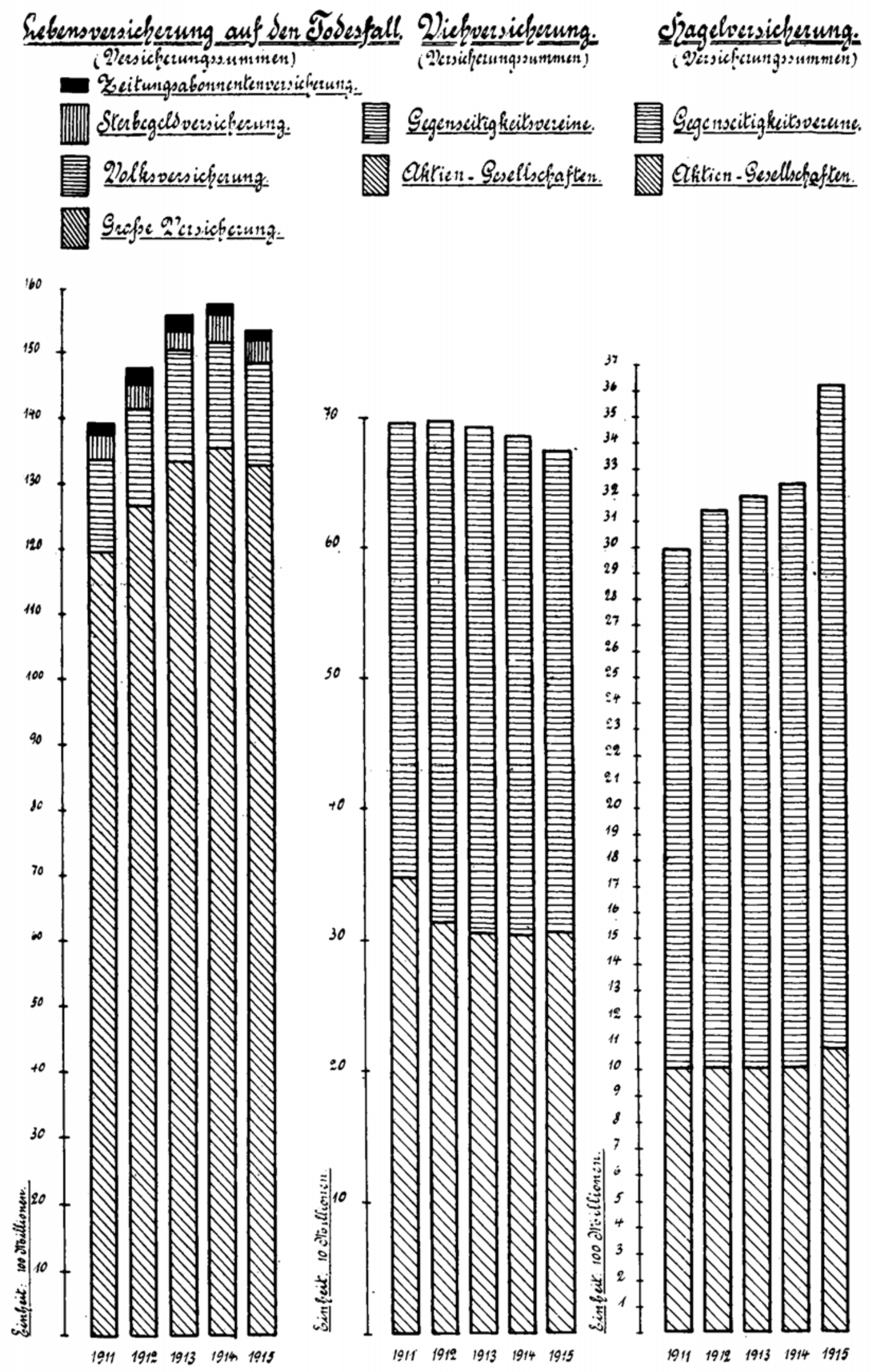

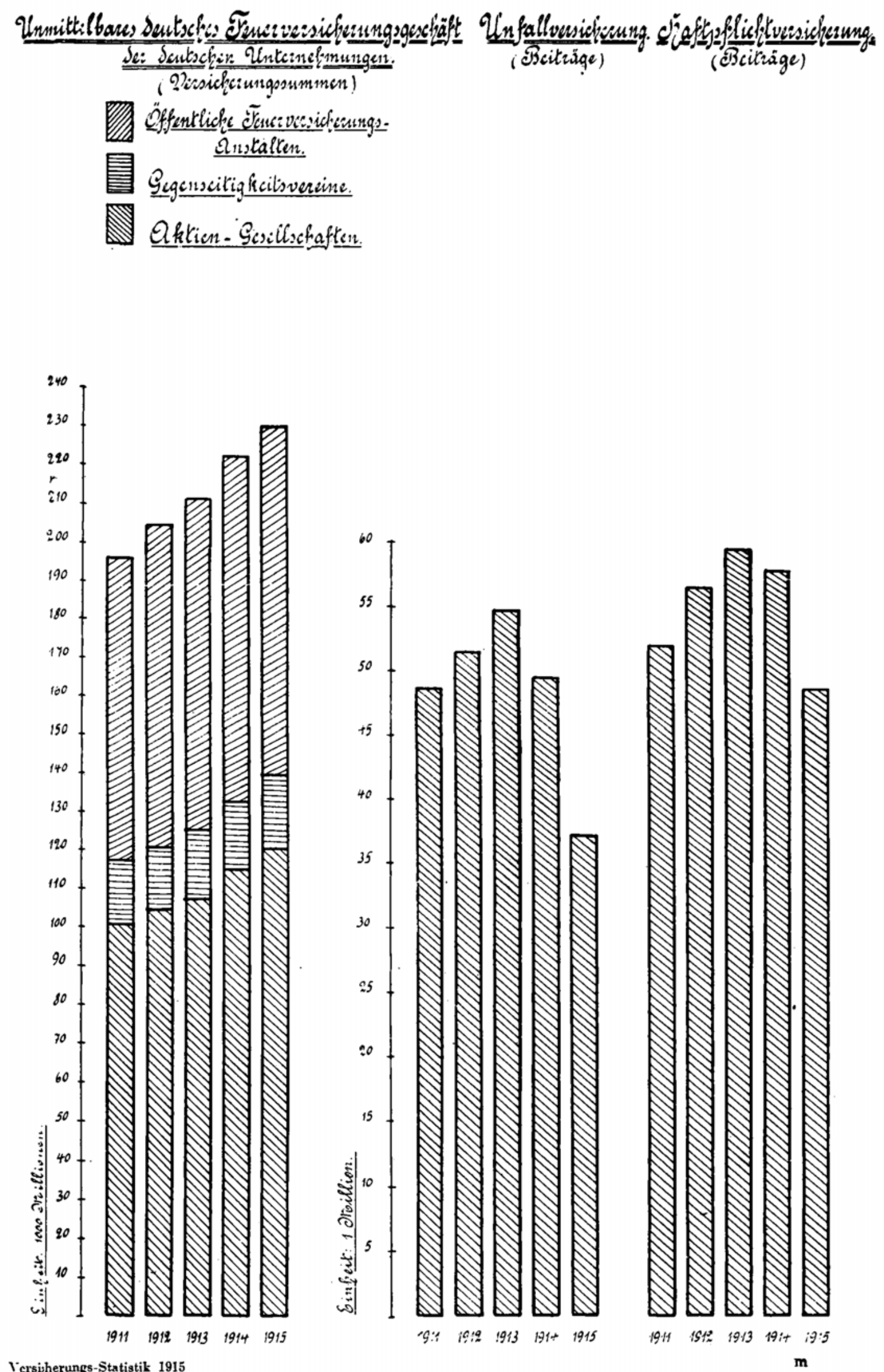
